\title{
Chemical and Biological Sensing with a Fiber Optic Surface Plasmon Resonance Device
}

\author{
by \\ Yanina Shevchenko \\ B Eng., Saint-Petersburg, Russia, 2004 \\ M. A. Sc., Carleton, Canada, 2007
}

A thesis submitted to the Faculty of Graduate and Postdoctoral Affairs in partial fulfillment of the requirements for the degree of

Doctor of Philosophy
in

Electrical and Computer Engineering

Ottawa-Carleton Institute for Electrical and Computer Engineering

Department of Electronics

Carleton University, Ottawa, Canada

(C) 2012

Yanina Shevchenko 
Library and Archives

Canada

Published Heritage

Branch

395 Wellington Street

Ottawa ON K1A ON4

Canada
Bibliothèque et

Archives Canada

Direction du

Patrimoine de l'édition

395 , rue Wellington

Ottawa ON K1A ON4

Canada
Your file Votre référence

ISBN: 978-0-494-94230-7

Our file Notre référence

ISBN: 978-0-494-94230-7
NOTICE:

The author has granted a nonexclusive license allowing Library and Archives Canada to reproduce, publish, archive, preserve, conserve, communicate to the public by telecommunication or on the Internet, loan, distrbute and sell theses worldwide, for commercial or noncommercial purposes, in microform, paper, electronic and/or any other formats.

The author retains copyright ownership and moral rights in this thesis. Neither the thesis nor substantial extracts from it may be printed or otherwise reproduced without the author's permission.
AVIS:

L'auteur a accordé une licence non exclusive permettant à la Bibliothèque et Archives Canada de reproduire, publier, archiver, sauvegarder, conserver, transmettre au public par télécommunication ou par l'Internet, prêter, distribuer et vendre des thèses partout dans le monde, à des fins commerciales ou autres, sur support microforme, papier, électronique et/ou autres formats.

L'auteur conserve la propriété du droit d'auteur et des droits moraux qui protege cette thèse. $\mathrm{Ni}$ la thèse ni des extraits substantiels de celle-ci ne doivent être imprimés ou autrement reproduits sans son autorisation.
In compliance with the Canadian Privacy Act some supporting forms may have been removed from this thesis.

While these forms may be included in the document page count, their removal does not represent any loss of content from the thesis.
Conformément à la loi canadienne sur la protection de la vie privée, quelques formulaires secondaires ont été enlevés de cette thèse.

Bien que ces formulaires aient inclus dans la pagination, il n'y aura aucun contenu manquant. 


\begin{abstract}
Fiber biosensors have emerged as an alternative to other optical sensor platforms which utilize bulkier optical elements. Sensors manufactured using optical fiber offer considerable advantages over traditional platforms, such as simple manufacturing process, small size and possibility for in situ and remote measurements. The possibility to manufacture a compact sensor with very few optical elements and package it into a portable hand-held device makes it particularly useful in many biomedical applications. Such applications generate a growing demand for an improved understanding of how fiber sensors function as well as for sensor optimization techniques so later these devices can suit the needs of the applications they are developed for.

Research presented in this thesis is focused on a development of a plasmonic fiber biosensor and its application towards biochemical sensing. The fiber sensor used in this study integrates plasmonics with tilted Bragg grating technology, creating a versatile sensing solution. Plasmonics alone is an established phenomenon that is widely employed in many sensing applications. The Bragg grating is also a well-researched optical component that has been extensively applied in telecommunication. By combining both plasmonics and Bragg gratings, it is possible to design a compact and very sensitive chemical sensor. The presented work focuses on the characterization and optimization of the fiber sensor so later it could be applied in biochemical sensing. It also explores several applications including real-time monitoring of polymer adsorption, detection of thrombin and cellular sensing. All applications are focused on studying processes that are very different in their nature and thus the various strengths of the developed sensing platform were leveraged to suit the requirements of these applications.
\end{abstract}




\section{Acknowledgments}

Work presented in this thesis was completed with enduring support and encouragement from my colleagues and collaborators. I would like to thank everyone who I had a chance to work with during years of my graduate studies.

First and foremost I would like to thank my supervisor Dr. Jacques Albert who I have worked with since I started the masters program and continued throughout my $\mathrm{PhD}$. Working with Dr. Jacques Albert has made a tremendous impact on my scientific foundation, work ethic, quality and direction of research. I am grateful to him for numerous opportunities, conversations and lessons that allowed me to become a better scientist. I am also thankful for granting me the research freedom to explore new applications, and for his wisdom and advice which helped me through a number of major decisions.

Furthermore, I would like to thank all members of my thesis committee including Dr. Hanan Anis, Dr. Hans Peter Loock, Dr. Maria C. DeRosa, Dr. Tom Smy and Dr. William Willmore for their feedback, reviews and all the effort that is going to be invested into finalization of this work.

I would also like to acknowledge my past and present colleagues at the Advanced Photonic Components Group and at the Department of Electronics at Carleton University. This includes Albane Laronche, Linguin Xiong, Rob Vandusen and Lyang Shao for all the help with the experimental work and manufacturing. I also would like to thank all my colleagues whom I have had a chance to work with while completing my $\mathrm{PhD}$ including 
Ksenia Yadav, Li-Yang Shao, Tuan Guo, Alexander Andreyuk, Nina Mamaeva, Zahirul Alam and Yang Zhang.

Most of the work presented in this thesis has been done in a close collaboration with several research teams from other laboratories and other Universities.

Dr. Maria C. DeRosa's team from Carleton University, Department of Chemistry has played pivotal role in the success of our aptamer biosensing project. Together with Dr. Maria C. DeRosa, David Blair and Tariq Francis, we worked on establishing the first biosensing experiments and tailoring the original experimental setup. I would like to thank Dr. Maria C. DeRosa and her team for the enthusiastic support and patience while proving us with an opportunity to explore various directions within this project.

I also would like to thank Dr. Anatoli Ianoul's team from Carleton University, Department of Chemistry for the help with the polyelectrolyte multilayer formation project. My acknowledgements go to thank Nur Ahamad and Graham Galway who have been of much help during this project.

Many thanks to Dr. Ali Khademhosseini and his team for their collaboration on a cellular analysis project. His colleagues Gulden Camci-Unal, Davide F. Cuttica, Daniel Aceves and Mehmet Dokmeci have contributed tremendously towards every aspect of the project. Working in Dr. Ali Khademhosseini' lab was an exceptional opportunity which has allowed me to expand my research skills and acquire valuable experience in the tissue engineering field.

I also would like to thank Dr. Tamiya for the opportunity to work with his group during NSERC-JSPS, a two-month summer program in Japan. It was an immensely useful experience to be a part of his team. It provided an opportunity to further my 
knowledge about biochemical sensors and learn new fabrication and experimental techniques. Many thanks to Le Quih Hoa, Mizuho Murahashi and Hiroyuki Yoshikawa for their help.

At last, I would like to offer my sincere gratitude to my family who have been a source of ongoing support and motivation through these years.

Funding for the work presented in this thesis came from few different sources including Natural Sciences and Engineering Research Council of Canada (NSERC), Canadian Institute of Photonics Innovation (CIPI), Japan Society for Promotion of Science (JSPS), Ontario Centres of Excellence (OCRI), Optical Society of America (OSA), LxSix and Carleton University (Department of Electronics and Graduate Studies). 


\section{Table of Contents}

Acknowledgements

List of Figures vii

Chapter 1 Introduction 1

1.1 Introduction into Biochemical Sensing 1

1.2 Fiber Biosensors $\quad 4$

1.3 SPR and Plasmonic Fiber Sensors 6

$\begin{array}{ll}\text { 1.3.1 SPR effect } & 6\end{array}$

$\begin{array}{ll}\text { 1.3.2 Plasmonic Fiber Sensors } & 10\end{array}$

1.4 Tilted fiber Bragg Grating SPR sensor (SPR-TFBG Sensor) 12

1.5 Data Extraction and Analysis Technique 13

1.6 Evaluation of a SPR Fiber Sensor's Performance 16

$\begin{array}{ll}1.7 \text { Biorecognition Schemes } & 22\end{array}$

1.7.1 Biosensing Principles $\quad 22$

1.7.2 Biosensing Structures and Modalities $\quad 24$

$\begin{array}{ll}1.8 \text { Cellular Analysis } & 27\end{array}$

1.9 Thesis Statement and Contribution $\quad 29$

1.10 Organization of the Thesis 31

Chapter 2 Polarization-selective grating excitation of plasmons in cylindrical $\begin{array}{ll}\text { optical fibers } & 39\end{array}$

Chapter 3 In situ monitoring of the formation of nanoscale polyelectrolyte coatings on optical fibers using Surface Plasmon Resonances 51

\section{Chapter 4}

4.1 Label-Free Biosensing with a Surface Plasmon Resonance Fiber Grating Aptasensor

4.2 Supplementary information

100

Chapter 5 Surface Plasmon Resonance Fiber Sensor for Real-Time and LabelFree Monitoring of Cellular Behavior

Chapter 6

128

6.1 Discussion $\quad 128$

6.2 Summary 131

6.2 Future Work Recommendations $\quad 135$

References (Chapters 1, 5 and 6) 141

Author's Contributions 145

Author's Research Output (2007-2012) 147

List of Abbreviations $\quad 148$

$\begin{array}{lr}\text { Glossary } & 149\end{array}$ 


\section{List of Figures}

Figure 1. Prism-based or Kretschmann configuration $\quad 8$

Figure 2. Proposed sensor structure utilizing gold-coated SMF-28 and 10 TFBG 12

Figure 3. Transmission spectra of SPR-TFBGs with $10^{\circ}$ of tilt immersed in water 14

Figure 4. Data analysis approaches 16

Figure 5. Biorecognition schemes 25 


\section{Chapter 1 Introduction}

\subsection{Introduction into Biochemical Sensing}

Scientific progress is managed through meticulous and reproducible work that includes such activities as hypothesizing, conducting experiments, measuring and analyzing. In order for knowledge to be objective, it is imperative that scientists are able to record and analyze processes that are being investigated. Research in biological sciences is difficult to imagine without the use of analytical devices that provide the opportunity to monitor and characterize various biochemical processes, which are not always visible to a human eye.

Today, monitoring of biochemical processes can be accomplished with a broad variety of analytical tools. They can be categorized based on the functionality as well as on the underlying physical principles employed for biochemical analysis. From a functionality point of view, there are imaging platforms such as microscopes, Atomic Force Microscope (AFM), Transmission Electron Microscope (TEM), Scanning Electron Microscope (SEM), analytical devices such as Surface Plasmon Resonance (SPR) systems ${ }^{1,2,3,4}$ evanescent-wave analyzers ${ }^{5,6,7,8}$ or Quartz Crystal Microbalance (QCM) sensing instruments ${ }^{9}$, spectrometers, Polymerase Chain Reaction (PCR) analyzers, optical and electrochemical sensors, just to name a few. From the employed physical phenomenon perspective, equipment can be categorized into groups of devices that rely on measurement of electrochemical phenomena, piezoelectricity ${ }^{9}$, ultrasound effect, 
interaction of electrons with media ${ }^{10}$ and the interaction of electromagnetic radiation with media. Optical tools are a major subgroup amongst these devices that measure the interaction of visible and IR electromagnetic radiation with studied biological substances or processes. Measured optical phenomena can consist of light attenuation, diffraction and reflection as measured by imaging and sensor tools, light absorption, emission and scattering as measured by spectroscopy tools, and luminescence as measured by fluorescence tools or they can be a combination of several optical effects ${ }^{11}$.

Optical imaging tools and optical analytical platforms are important subgroups within the optical devices category that provide an opportunity to perform very precise and highly accurate measurements. The measurement modes can be real-time, labeled or label-free and can be tailored to measure hard to reach processes. While providing the possibility to measure biochemical processes at very high temporal and spatial resolution, imaging devices (like many other analytical tools) are often complex, require trained personnel for their operation and cannot be applied outside laboratory setting. Another limitation is associated with the fact that optical imaging is restricted to capturing processes occurring on a surface (such as on skin, the surface of a glass slide or on an optical chip); it is impractical and often not possible to use such tools to analyze processes occurring deep within a volume. This limitation arises from the fact that electromagnetic radiation at optical wavelengths and further into IR is attenuated by propagation through the biological samples and solids ${ }^{12}$. Even though there are efforts aimed at developing imaging tools for in vivo applications such as "microscope on a needle" by D. Samson ${ }^{13}$, such tools are still very rare and there is a great demand for more solutions that could be applied for in situ and in vivo studies. 
The biomedical sensor field has been developing complimentary to imaging and other traditional laboratory devices fields. It has been driven primarily by a demand for compact and affordable analytical tools that can be applied in outside of laboratory setting and in an environment with limited resources. Despite the availability of a great variety of analytical tools, most of them can only be used in a laboratory setting due to their cost, size and complicated operation routine. In this context, miniaturized sensing devices provide an opportunity to measure reactions that would normally be measured in a laboratory setting but without a need for expensive equipment or advanced knowledge about how the equipment operates. Examples of point of care optical sensors successfully applied towards biomedical analysis include the hand-held optical device from Phillips Electronics that can measure an analyte at concentrations below the 1 picomolar level within first 5 minutes $^{14}$.

Besides the demand for compact and cost-effective sensor solutions, there is also a growing demand for analytical tools that can be integrated into relatively complex and difficult to reach environments and used for in vitro applications. For instance, a group of fluorescent fiber sensors have been integrated with bioreactors and used for measurement of glucose and oxygen levels during fermentation process ${ }^{15}$. Another example is a group of sensors that are developed for in vivo applications. Particularly, it was proposed to manufacture optical biosensors using biodegradable and bioabsorbable materials such as silk fibroin ${ }^{16}$ or salmon DNA ${ }^{17}$ therefore the sensors would have a potential be implanted and used for in vivo analysis.

In the case of these applications, the sensors' size, cost, integrability and environmental compatibility are the most important factors that determine the success of 
the sensor's application. Even though performance characteristics such as bulk chemical sensitivity, limit of detection (LOD), signal to noise ratio (SNR) and other performance indicators still play a major role, sensor integration capacity and compatibility with different environments have been becoming more and more important over the past years.

In addition to developing an appropriate sensing modality and finding a sensor configuration that fits each particular application, there is also a considerable amount of work aimed for optimization, integration and packaging of optical biosensors. It includes optimization of a biorecognition scheme therefore it would maximize and enhance sensor's capability to detect the biological target. Moreover, it also involves integration of the sensor element with a microfluidic platform and their packaging into a hand-held device $^{18}$.

\subsection{Fiber Biosensors}

A biosensor can be defined as a "compact analytical device or unit incorporating a biological or biologically derived sensitive 'recognition' element integrated or associated with a physio-chemical transducer"19. The function of the transducer is to translate signal from the output domain of the recognition system to the optical domain ${ }^{20}$. Fiber biosensors present a group of optical sensors whose transducing elements are manufactured using different kinds of optical fiber. 
Optical fiber is a circular optical waveguide that was invented for the purpose of light transmission ${ }^{21,22}$. One of the major objectives behind the original fiber design was to reduce the optical attenuation and scattering losses which is required for delivering of optical signals over long distances. To archive it the fiber was designed so that it consisted of a circular core made of silica enclosed by a larger cladding layer that confined the light propagating in fiber within its core. Such configuration provides low attenuation of $0.20-0.22 \mathrm{~dB} / \mathrm{km}^{23}$ which is suitable for long-haul communications. Significant progress in material science, development of new fiber manufacturing techniques and design of novel fiber structures have made it possible to use optical fiber in a set of new applications including biochemical sensing.

Transducing sensor elements manufactured using optical fiber offer a set of advantages over other optical sensors. First and foremost, fiber sensor elements can be very compact thus can be easily applied for a range of in situ applications. They also can be easily integrated into fiber optic communication systems. This eliminates the need for additional optical or electro-optical components that would normally be required for integrating sensors into a fiber optic system. Additionally, a fiber-based sensor provides the opportunity for remote sensing as well as an opportunity for multiplexing of several sensing elements ${ }^{24,25}$.

The most common sensing modalities that are currently being used in combination with optical fiber for biochemical sensing are fluorescence ${ }^{26}$, evanescentwave effect $^{27}$ and Surface Plasmon Resonance effect ${ }^{28,29,30}$. Other recently explored modalities include Raman scattering ${ }^{31}$ and Surface enhanced Raman scattering ${ }^{32}$. In all these cases light propagating through optical fiber interacts with the analyzed 
biochemical substance or process, and as a result of this interaction some characteristics of light are being altered. It can include change of phase, change of intensity or wavelength shift. In the case of fluorescence, light propagating through a fiber interacts with fluorophore molecules on its surface and light emitted by the fluorophores can be used as the output of the sensor system. In the case of the evanescent wave sensors light propagating inside fiber leaks outside and interacts with the analyzed matter. Due to this interaction light experiences attenuation, monitoring of this process can be used to interpret chemical reactions that are being measured. In the case of SPR, light travelling within fiber is used to excite SPR on its surface and coupling to SPR is used to track chemical changes. In case of Raman scattering, light propagating within fiber (regular or PCF) is used to excite Raman scattering and Raman signal is being monitored as sensor's output.

In all these cases, independently of the sensing modality, there is a necessity to select an appropriate fiber configuration that would allow for the most effective utilization of the sensing mechanism and most sensitive analyte detection.

\subsection{SPR and Plasmonic Fiber Sensors}

\subsubsection{SPR effect}

SPR is a well-known and well-researched electromagnetic phenomenon that was

first discovered in 1950s by R. Ritchie ${ }^{33}$. Ritchie observed attenuation of electronic 
radiation passing through metal films that was attributed towards collective oscillations of electron plasma in the metal. Surface plasmons exist on a surface of metals or highlydoped semiconductors ${ }^{34}$ surrounded by a dielectric. This phenomenon has dual electronphoton nature and can be used to convert light into plasmonic waves and vise-versa. Excitation of surface plasmon waves can be accomplished using light as well as electron beams.

SPR excitation using light is most commonly associated the Kretschmann configuration (Kretschmann and Raether, 1968) where SPR is excited on the flat surface of a gold-coated dielectric prism (Fig.1). The configuration employs a high refractive index prism with a thin metal layer on the prism's bottom. Light propagating in the prism is reflected at the interface between prism and metal by means of the total internal reflection mechanism. Evanescent field of the reflected light at the first interface penetrates into the metal and reaches the second metal-dielectric interface. If the refractive index of the second dielectric is smaller than the refractive index of the prism and also, if the propagation constant of light propagating in the prism matches the propagation constant of the surface plasmon wave, then a surface plasmon resonance can be excited. 


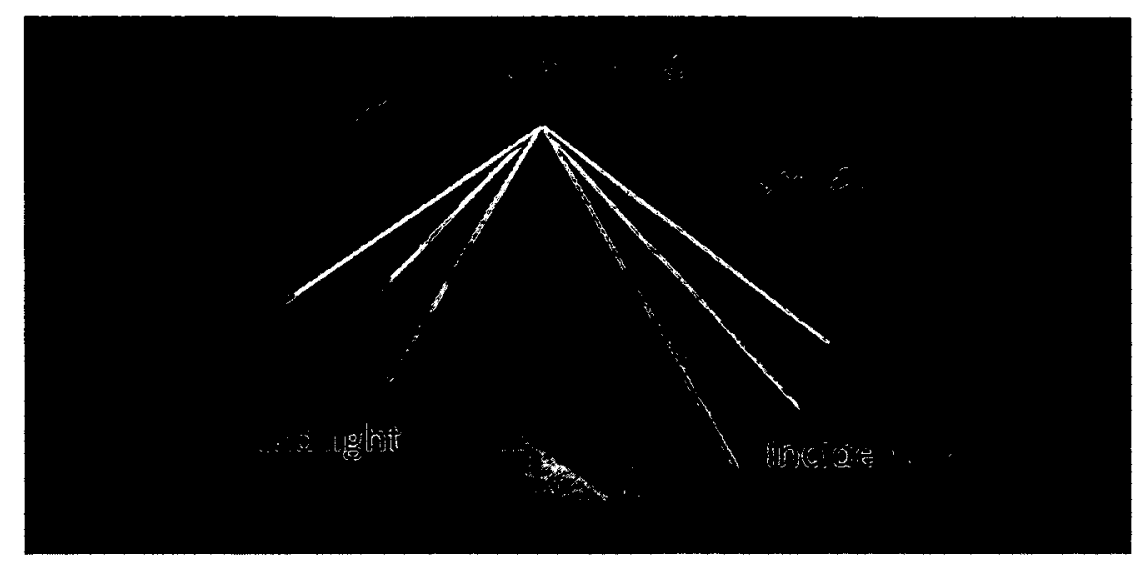

Figure 1. Prism-based or Kretschmann configuration.

As mentioned above, the requirement for the excitation of the plasmon wave is the phase matching between wave vector of incident light and wave vector of plasmonic wave propagating on the outer surface of the metal ${ }^{35}$. The wave vector of light incident upon bottom of the prism can be found as:

$K_{1}=\frac{2 \pi}{\lambda} \sqrt{\varepsilon_{p}} \sin (\theta)$

The wave vector of the plasmon wave propagating on the metal's surface can be found as:

$$
K_{2}=\frac{2 \pi}{\lambda} \operatorname{Re}\left\{\sqrt{\frac{\varepsilon_{m} \varepsilon_{d}}{\varepsilon_{m}+\varepsilon_{d}}}\right\}
$$

Then the phase-matching condition for the prism configuration can be found as:

$$
K_{1}=K_{2} \quad \text { or } \quad \sqrt{\varepsilon_{p}} \sin (\theta)=\operatorname{Re}\left\{\sqrt{\frac{\varepsilon_{m} \varepsilon_{d}}{\varepsilon_{m}+\varepsilon_{d}}}\right\} \text {, }
$$

Where $\varepsilon_{p}$ is the dielectric permittivity of the prism, $\theta$ is the angle of incidence of light, $\varepsilon_{d}$ is the dielectric permittivity of the material adjunct to the surface of the metal (sensed 
medium), $\varepsilon_{m}$ is the dielectric permittivity of the metal, $\lambda$ is the wavelength of the incident light.

Monitoring of a chemical process occurring on the surface of the metal-coated prism can be accomplished by monitoring of some of the parameters of light reflected off the base of the prism, particularly by measuring reflected light's intensity or angle at a fixed wavelength or by measuring intensity or wavelength at a fixed angle.

Today SPR has found its applications in many applied and fundamental research areas including solar cells, SERS, lasers, metamaterials, integrated opto-electronic circuits and sensing. First applications of SPR platform in sensing have been reported almost three decades ago $^{36}$ and today SPR prism-based platforms have established themselves as a highly sensitive and reliable analytical tool. Wide application of the SPR effect in biosensing can be attributed to the relatively high sensitivity toward refractive index change, small depth of penetration of plasmon field into sensed medium and thus possibility to measure chemical changes happening at the sub-micron scale as well as to the label-free character of the analysis. Sensitivity towards refractive index change for SPR prism configuration is estimated to vary between 970 and $13800 \mathrm{~nm}$ per Refractive Index Unit $(\mathrm{RIU})^{36}$ dependently on the wavelength used to excite SPR. Such high sensitivity combined with a small depth of penetration of plasmon field inside adjunct dielectric media enables detection of target biomolecules at $\mathrm{ng} / \mathrm{ml}$ and sub-ng/ml concentrations ${ }^{37}$. 


\subsubsection{Plasmonic Fiber Sensors}

The excitation of SPR effect on the surface of an optical fiber is more challenging than the excitation of SPR on the planar waveguide due to several factors.

First, there is a need to achieve phase matching between light propagating in a fiber and the SPR wave on the surface of the fiber.

Second, light propagating in a standard single mode fiber is confined to the fiber's core and cannot reach the surface of the cladding where metal coating would normally be deposited. Thus there is a need to alter fiber's geometry in order to achieve overlap between light propagating in the core and metal coating on a surface of the fiber.

Most of the SPR fiber sensor configurations fall into one of two groups. The first group of sensors is implemented using custom-made fibers while the second group of sensors relies on in-fiber gratings for excitation of SPR. Custom-made fibers, such as tapered fiber ${ }^{38}$, bent and polished fiber ${ }^{39}$, or a fiber with a partially removed cladding 40,41, D-type fiber ${ }^{42}$, metalized photonics crystal fiber $^{28}$ are all examples of fiber with geometry being tailored or designed for the purpose of SPR excitation. In these cases metal layer is deposited in a proximity to light propagating in the fiber's core or cladding. Manufacturing of custom-made fiber such as metalized photonics crystal fiber might be challenging and costly for mass production. On another hand alteration of commercially available fiber's geometry in a laboratory setting does not provide very precise control over new fiber geometries thus leading towards poorly controlled SPR excitation. Variation of fiber's parameters such as thickness of the cladding, proximity of gold layer 
to the core can have tremendous effect on the quality of SPR excitation as well as hinder sensor's reproducibility.

Fiber gratings on another hand accomplish phase matching between SPR and light propagating in a fiber, sometimes without alteration of optical fiber's geometry. Gratings can be used to excite fiber modes with effective index matching effective index of SPR mode thus allowing for very effective phase matching. Examples of fiber gratings applied for SPR excitation include fiber Bragg grating $(\mathrm{FBG})^{43}$, long period grating (LPG) ${ }^{44}$, tilted fiber Bragg grating (TFBG) ${ }^{45}$ and surface relief gratings ${ }^{46}$. Generally, use of the fiber gratings can lead towards less complicated manufacturing procedures with significantly better control over the SPR excitation mechanism. However, use of a straight grating such as FBG is not sufficient for excitation of SPR in a single mode fiber because it does not allow for excitation of mode that could potentially reach the surface of the cladding. Use of FBG requires combination with altered fiber geometry as it was proposed by G. Nemova and R. Kashyap (2007) ${ }^{47}$. On another hand both LPG and TFBG allow for excitation of cladding modes that could be used to excite SPR in a single mode fiber without need to change its geometry. Both kinds of gratings provide an opportunity to design plasmonic sensors using a single mode fiber while fully preserving fiber's geometry. This thesis will examine work of a fiber sensor that relies of TFBG for plasmonic excitation. Sensor's principle of is outlined below as well as in Shevchenko et al $(2007)^{45},(2010)^{48}$. 


\subsection{Tilted Fiber Bragg Grating SPR sensor (SPR-TFBG Sensor)}

The fiber sensor studied in this thesis relies on a tilted Fiber Bragg Grating as a coupling element for excitation of SPR effect (Fig. 2). This configuration has been first presented in Shevchenko et al $(2007)^{45}$ and described in more details in Shevchenko et al $2010^{48,49}, 2011^{30}$, Shao et al $(2010)^{50}$ and Caucheteur et al (2011) ${ }^{51}$.

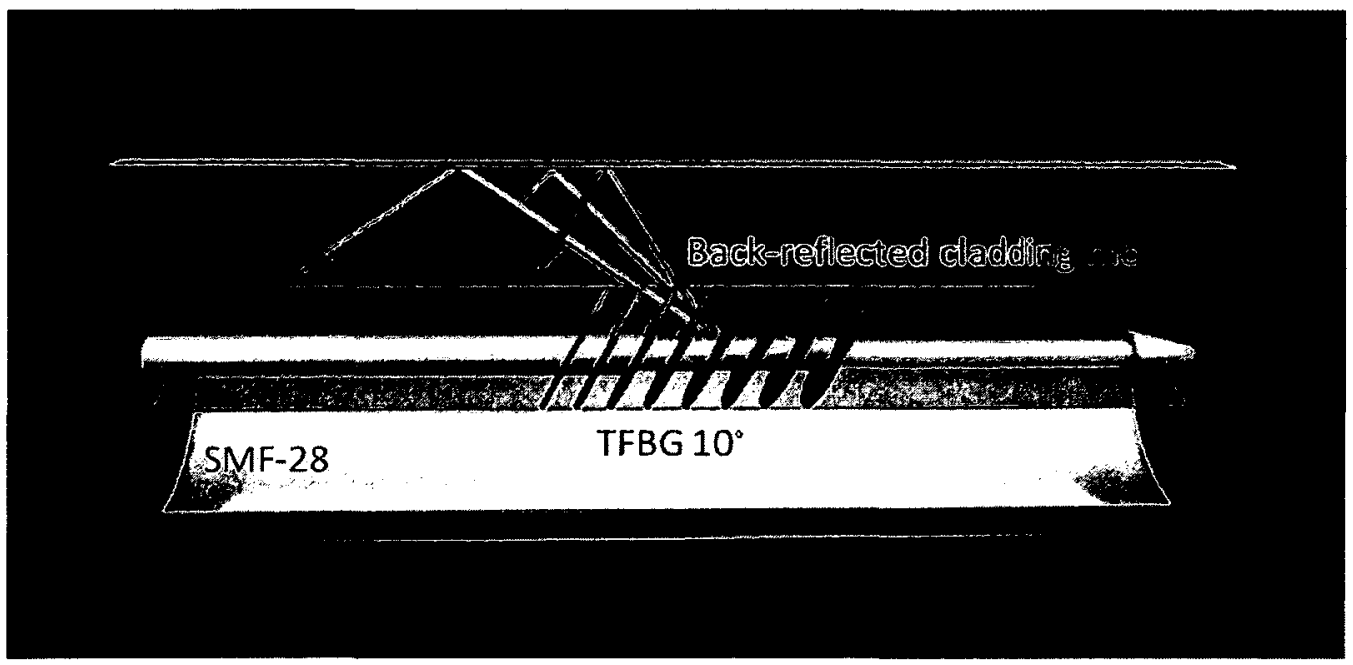

Figure 2. Proposed sensor structure utilizing gold-coated SMF- 28 and $10^{\circ}$ TFBG.

Presented sensors configuration consists of a single-mode fiber (SMF-28) that has TFBG imprinted in its core and a gold coating on the surface of the cladding. TFBG acts as a resonant element that couples core mode into a group of back-reflected cladding modes. Tilt of the grating which regularly does not exceed $10^{\circ}$ for biosensing applications allows to access high order claddings modes that reach out to the surface of the cladding. Coupling into SPR wave occurs if the effective index of a cladding mode and polarization state matches those parameters of the plasmon wave on the surface of the gold coating. 
Tilt of the grating offers control over which cladding modes could be accessed and thus it could be used to alter operating range of the chemical sensor. Sensor configuration presented in Shevchenko et al $2007^{45}$ had a tilt of $6^{\circ}$ and was applied to work in high refractive index liquids (1.3967 to 1.4442$)$, while sensor presented in Shevchenko et al $2010^{30}$ had a tilt of $10^{\circ}$ and thus was applied to work in water solutions (1.3324). While providing an opportunity to reach cladding modes with very specific effective indexes, tilted gratings also offer an advantage of an access to several tens of cladding modes the same time. It provides an opportunity for the same sensor to be applied in different environments with different refractive indexes, a property that is difficult to be achieved for sensors employing straight fiber gratings that access only one mode the same time. Furthermore, excitation of a big number of cladding modes along with a backpropagating core mode provides a possibility to eliminate temperature cross-sensitivity during post-processing of sensor's data ${ }^{50}$.

\subsection{Data Extraction and Analysis Technique}

The SPR-TFBG sensor has a relatively complex output signal; transmission spectrum of such sensor includes a back-reflected core resonance (Bragg resonance), a ghost resonance that is positioned very close to the Bragg resonance and a set of back-reflected claddings modes with some of them being coupled to SPR. Fig. 3 (A) shows a typical response of a SPR-TFBG with $10^{\circ}$ of the tilt that was measured in transmission regime. 

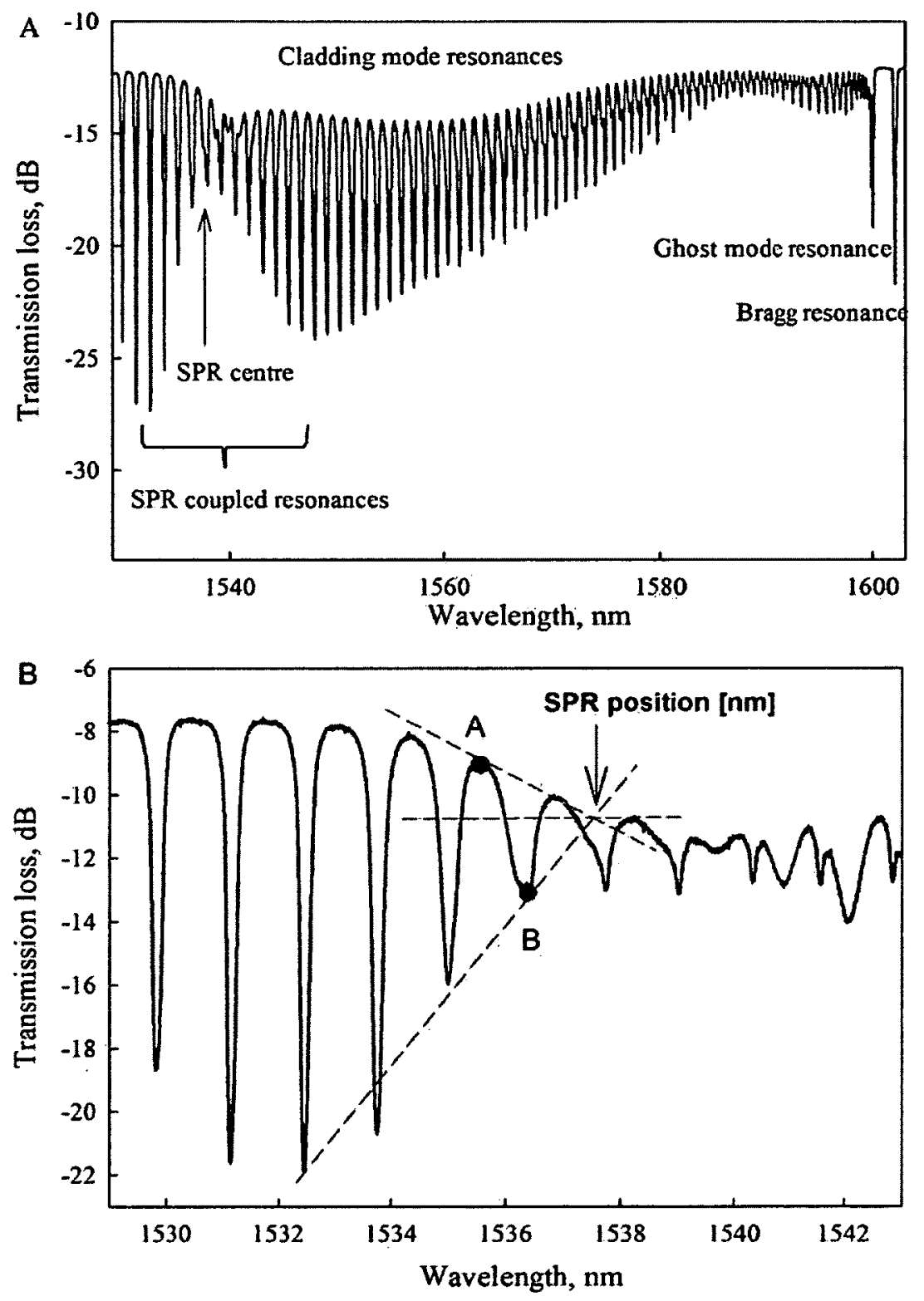

Figure 3. Transmission spectra of SPR-TFBGs with $10^{\circ}$ of tilt immersed in water. (A) Full spectrum illustrating Bragg resonance as well as all cladding mode resonances. (B) Part of the spectrum coupled to SPR that was a subject to several analysis methods. Intersection of two tangential slopes shown with red color is used to identify wavelength position of SPR. 
Excitation of the SPR effect on the surface of the gold-coated TFBG results in several cladding modes being coupled to SPR simultaneously. Transmission spectrum of gold-coated TFBG usually exhibits well-pronounced SPR effect in a form of a group of cladding modes having smaller amplitude and the spectrum having 'bottle-like' shape. This effect is shown in the Fig.3 (A), (B). SPR-coupled modes are indicated with a red arrow and a red bracket. Coupling of a cladding mode into SPR results in smaller amplitude of the cladding resonances. This effect can be attributed to the tunneling of cladding modes across the metal layer, their subsequent coupling into SPR wave and absence of total-internal reflection at the cladding-metal interface that is responsible for production of pronounced back-reflected resonances ${ }^{48}$.

The analysis of the experimental data should consists of tracking of the spectral changes occurring in the SPR-coupled part with the purpose of extracting information pertaining to the chemical changes taking place on the surface of the gold-coated fiber. Analysis of bulk refractive changes of a bigger amplitude, as reported in Shevchenko et al $2007^{45}$ and Shevchenko et al $2010^{49}$, is relatively simple to conduct. It could be done by tracking position of SPR-coupled part of the spectrum by fitting it with a suitable shaped function ("a second order polynomial over the 4 central resonances for instance" ${ }^{49}$ ). However the application of the sensor towards monitoring biochemical reactions does not always result in significant wavelength shift of the SPR-coupled part and thus requires analysis method that would permit interpretation of very fine spectral changes.

For the purpose of identifying of the most suitable data analysis approach, several analysis methods were proposed and applied towards the interpretation of the experimental data. The suitability of the proposed analysis techniques were evaluated by 
comparing the results from the measured data to the anticipated results and the SNR level. These data analysis approaches are presented in Fig.4.
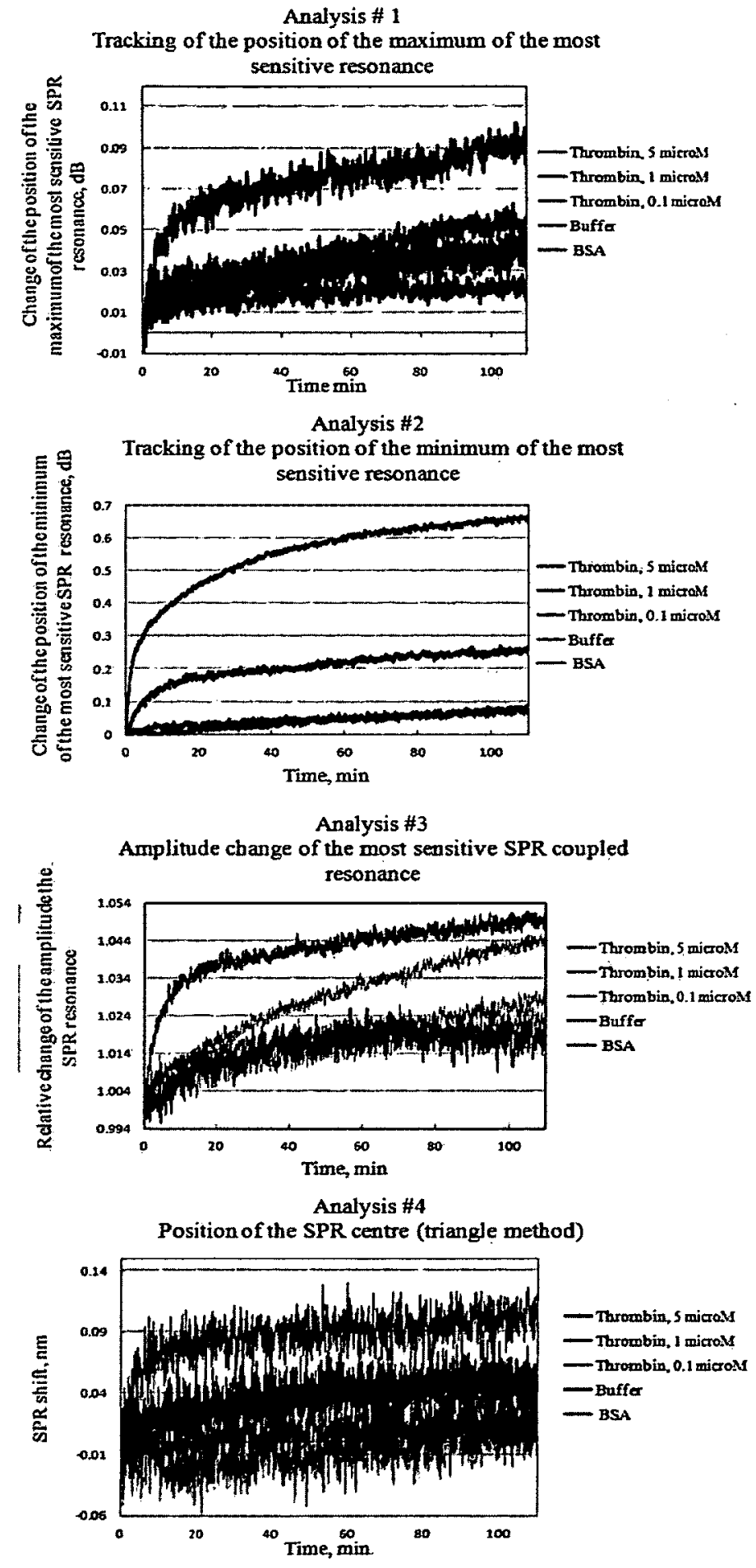

Figure 4. Data analysis approaches. 
Data presented in Fig.4 was collected from five different sensors that were used in experiments with thrombin protein. Each sensor prior to immersion in a solution with thrombin was functionalized with a layer of aptamers that were supposed to selectively interact with thrombin protein ${ }^{30}$. Three sensors were applied to detect thrombin at concentrations of $5 \mu \mathrm{M}, 1 \mu \mathrm{M}, 0.1 \mu \mathrm{M}$. Two more sensors were applied to measure SPR response in absence of thrombin, one was tested in buffer and another one was tested in BSA solution. BSA solution stands for Bovine serum albumin, a protein that was not supposed to interact with aptamer molecules but could adsorb to the surface to the sensor due to other background processes (non-specific adsorption). Both tests in buffer and in BSA were added as controls illustrating sensors response in absence of target, possibly with some non-specific background signal.

Analysis \#1 was based on tracking of the position the maximum of the most sensitive SPR-coupled resonance (point A on Fig. 3(B)). Sensor's signal was plotted as position of maximum $A(t)$ versus time $t$. Analysis \#2 was based on tracking of the value of the minimum of the most sensitive SPR-coupled resonance (point B on Fig. 3(B)). Sensor's signal was plotted as position of minimum $B(t)$ versus time $t$. Analysis \#3 was based on tracking of the relative change of the amplitude of the most sensitive SPRcoupled resonance. Sensor's output was found as relative change of the amplitude $\frac{A(t)-B(t)}{A_{t=1}-B_{t=1}}$ versus time $t$. Analysis \#4 was based on the identification of the wavelength position [nm] of the SPR coupled part of the spectrum and tracking its shift over time $t$. Th position of the SPR centre was found as intersection of the two tangential slopes drawn over the upper and lower part of the spectrum (Fig. 3(B)). Both slopes were drawn 
over the top and the bottom of the most-sensitive resonance (points A and B) as well as over another resonance with slightly bigger amplitude.

Although all applied analysis methods could be used to interpret spectral changes as they all reflect on the transition of the SPR-coupled part of the spectrum, it was concluded empirically that analyses \#3 and \#4 were the most accurate. In absence of other analytical devices that could serve as a reference during such an experiment, relation between reported SPR signals and applied concentrations of target molecule was the deciding factor. Furthermore, SNR in the case of analysis \#3 was smaller than SNR in the analysis \#4 that lead to application of analysis \#3 towards all data presented in the following chapters.

It should be noted that even though theoretical analysis was applied to identify SPRcoupled modes and predict their change, proposed and applied data analyses techniques were mostly developed as a result of interpretation of experimental findings.

\subsection{Evaluation of a SPR Fiber Sensor's Performance}

Performance of a fiber sensor similarly to any chemical sensor can be evaluated using a few characteristics including sensitivity towards refractive index change, SNR and resolution.

1) Sensitivity of a SPR sensor can be found as a derivative of the monitored SPR parameter with the respect to the parameter to be determined (refractive index) ${ }^{36}$. In the case of the spectrally interrogated sensors SPR sensors, monitored SPR parameter is 
wavelength position of the SPR coupled region. Thus sensitivity $S$ can be found as wavelength shift $\delta \lambda_{s p r}$ per refractive index change $\delta n$ :

$$
S=\frac{\delta \lambda_{s p r}}{\delta n}
$$

As a rule, SPR sensitivity is reported as $\delta \lambda_{s p r}$ per RIU that reflects on how much SPR position shifts when refractive index of media in which sensor is immersed changes by 1 . Sensitivity of the sensor presented in this study to changes in the bulk refractive index was found to be $555 \mathrm{~nm}$ per $\mathrm{RIU}^{49}$.

2) Signal to Noise Ratio (SNR) reflects on how accurately sensor can measure certain process. If sensor's output is analyzed using relative amplitude change method then SNR can be determined as:

$S N R=\frac{\delta A_{s p r}}{\delta A_{n}}$

where $\delta A_{s p r}$ is change of the amplitude during an experiment, $\delta A_{n}$ is noise level associated with particular resonance being measured. While $\delta A_{n}$ is a parameter that strongly depends on the performance of the interrogation system, $\delta A_{s p r}$ completely depends on the process to be measured, thus SNR can vary from an application to an application.

3) Resolution is another important parameter that can be found as the minimum change in the parameter to be determined that can be recorded by a sensor and resolved during data analysis ${ }^{36}$. In the case of a chemical sensor the resolution is the smallest refractive index change $(\delta n)$ that can be recorded. Resolution depends on the properties of the plasmonic interrogation system such as noise level of the source, SPR width, 
detector's resolution. Small resolution can hinder sensor's capability towards measuring certain chemical reactions even if the sensitivity of that sensor is relatively high and permits the measurement. Resolution of the sensor presented in this study was found to be $4 \times 10^{-549}$.

4) Operating range or dynamic range of a chemical sensor is the range of refractive index values $\Delta n$ that can be measured by a given sensor configuration. In the case of a fiber SPR sensor dynamic range can be an issue if there is only one or few modes can be coupled to SPR by the same sensor. In that case dynamic range of refractive indexes to be measured will be limited by the spectral width of the resonances to be coupled into SPR. In the case of a sensor utilizing tilted grating there are many cladding modes that could be coupled into SPR therefore providing the sensor with a relatively wide operating range. Current configuration allows for measurement of SPR shift within $1525 \mathrm{~nm}-1580 \mathrm{~nm}$ range, this in combination with chemical sensitivity of $555 \mathrm{~nm} / \mathrm{RIU}$ provides SPR-TFBG sensor with operating range of $\Delta n=0.1$.

In addition to the mentioned above parameters, work of a biosensor is a subject to a few others estimations such as limit of detection (LOD), specificity and the capability for evaluating the affinity of biomolecules.

5) Limit of detection (LOD) is the smallest quantity of analyte that can be distinguished by a sensor ${ }^{52}$. LOD can be found as:

$$
L O D \cong 3 \sigma B,
$$

where $\sigma B$ is noise magnitude (that could be found as a standard deviation). In other words "LOD corresponds to the analyte amount for which the signal-to-noise ratio is equal to $3^{, 52}$. Since SNR is in a direct relation to the process to be measured, LOD is not 
only a function of interrogation system and noise generated by the system but also depends on the kind of analyte to be detected.

6) Specificity or selectivity is the capability of a biosensor to detect presence of the analyte and distinguish it from other biomolecules that could be presented in same solution. This parameter is usually pre-determined by the biorecognition scheme $e^{53}$ and by the affinity of the biorecognition element towards the analyte.

Besides concentration measurements sensors and analytical tools can be applied towards estimation of affinity $\mathrm{Ka}$ of one biomolecule towards another. Affinity $\mathrm{Ka}$ can be estimated through $\mathrm{Kd}$ which is equilibrium dissociation constant:

$K_{a}=1 / K_{d}$,

Evaluation of $K a$ and $K d$ constants requires a set of experiments during which a sensor would be exposed to analyte solutions of varying concentrations. $K d$ then could be found as a concentration at which sensor reports $50 \%$ of the saturation signal.

\subsection{Biorecognition Schemes}

The biorecognition scheme is an important element of the biosensor platform as this has a direct impact on the output of a biosensor and its ability to be applied to the measurement of biochemical reactions. The function of the biorecognition element is to interact with the target in a way that will introduce a certain measurable change on the surface of the transducer. It could be a change in the thickness of the biorecognition 
layer, a change of its refractive index, or any other changes that could be later translated into optical signal.

\subsubsection{Biosensing Principles}

There are several classes of biorecognition schemes that serve different kinds of biosensors. Most biosensors use the following types of biorecognition principles:

\section{1) Enzyme based recognition:}

Enzymes interact with a target and catalyze its conversion into different molecules. As a result of such a conversion, newly formed molecules can produce a signal that can be translated into the output of the sensor or could be detected by the sensor. Examples include the conversion of target molecules into molecules with a specific adsorption band, or causing target molecules to fluoresce ${ }^{12,15}$.

\section{2) Antibody based recognition (Fig. 5 (A)):}

This approach takes advantage of antibodies that have high affinity and selectivity towards their targets'-antigens. Antibodies are "highly-soluble serum glycoproteins involved in the defense mechanism of the immune system" ${ }^{254}$. An antibody recognizes its antigen based on the antigen's molecular shape as well as its fit with regards to the antibody's molecular shape, somewhat analogous to the way a key fits its lock ${ }^{55}$. The number of antibodies that could be utilized for detection of antigens was increased following Kohler and Milstein's development of a monoclonal antibody production technique that allows for generation of an antibody for a broad range of antigens ${ }^{56}$. Similar to enzyme-based recognition, antibody-antigen binding events can be translated 
into optical signals through changes in the properties of the biorecognition layer (its density, refractive index or thickness) or by an addition of a fluorescent marker, radioisotope or another labeled antibody (Fig. 5 (B)).

\section{3) Aptamer based recognition:}

Aptamers are short single-stranded oligonucleotide sequences that possess unique binding characteristics to their targets ${ }^{57}$. Aptamers are engineered through an in vitro selection process known as SELEX (Systematic Evolution of Ligands by Exponential Enrichment). Typical length of aptamer sequences is $15-80$ bases (with molecular weight of 6-26 $\mathrm{kDa}$ ) that makes them smaller than other biorecognition elements such as antibodies ${ }^{58}$. Binding affinities of aptamers depend on the target and can vary from picomolar to a high molar scale $\left(10^{-7} \mathrm{M}\right)^{57}$. Target recognition is based on very specific matching between aptamer sequences, their 3D structure and targets. For example 15base aptamer synthesized to bind with thrombin recognizes molecules of thrombin by forming a G-quadruplex structure with the thrombin, which normally would not be formed in a solution without thrombin ${ }^{59}$. Formation of complicated aptamer-target complexes can be governed by electrostatic and hydrophobic interactions ${ }^{59}$.

Overall, aptamers have emerged as a strong alternative to antibody-based biosensing due to a few advantages. First, aptamer in vitro SELEX production is more cost effective and results in less batch-to-batch variation than the in vivo monoclonal antibody production process ${ }^{60}$. SELEX allows for isolation of new oligonucleotide sequences that can bind to a very broad range of targets including complimentary oligonucleotide sequences, proteins, enzymes, viruses and bacteria. Furthermore, aptamers, unlike antibodies, are more robust can bind to drugs and toxins and also can be 
handled at room temperature ${ }^{61}$. Moreover, if aptamers are used in assays, their conjugation with labels does not affect their affinity towards their targets ${ }^{60}$.

\subsubsection{Biorecognition Structures and Modalities}

Independent from the employed biorecognition element, biosensing schemes can have relatively straightforward structures where a target interacts with only one kind of biorecognition element (Fig. 5(A)), or they can have more complex structure that involve several kinds of molecules interacting with the target. In the latter case, the biorecognition scheme can not only have several different kinds of biomolecules interacting with the target, but it also can involve a variety of nanostructures. The main purpose behind such schemes is to achieve amplification of the detection signal. Amplification is yielded by the addition of a label that increases the measured signal. Depending on the sensing mechanism, amplification can be implemented by addition of fluorophores, nanoparticles or radioactive elements. ELISA (enzyme-linked immunosorbent assay) is an example of such a structure that relies on two additional antibodies and a tag (usually fluorophore) for the detection of an antigen (Fig. 5(B)). As a rule, sandwich assay schemes yield better LOD. However, performance of these schemes are intrinsically more complicated, costlier due to the number of involved sensing elements and also result in higher chances of false-positive and false negative responses. 

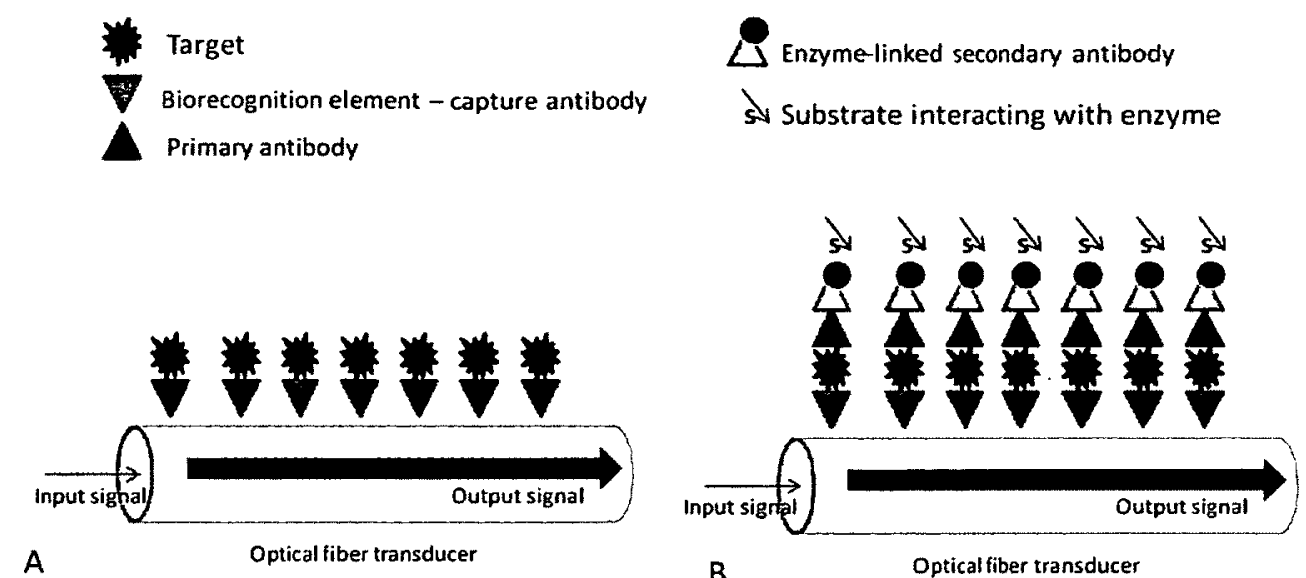

B

Optical fiber transducer

Figure 5. Biorecognition schemes. (A) Simple biorecognition scheme consisting of a biorecognition element (capture antibody) and a target. (B) ELISA immune assay, also known as "capture assay"62 consisting of a capture antibody, a target, a primary antibody, a secondary antibody conjugated with an enzyme, and a substrate that produces a fluorescent signal upon interaction with the enzyme.

The mentioned above biorecognition schemes can be classified into labeled and label-free approaches, depending on their use of fluorophores or other kinds of tags. Label-free biosensing traditionally relies on the detection of an analyte or the targeted process only with the help of a biological or chemical receptor ${ }^{63}$ and without use of a fluorophore or any other tag that could facilitate analyte detection. Label-free biorecognition is more advantageous than labeled detection due to the fact that it does not interfere with the measured process. The addition of a label affects the receptor-analyte dynamics and can hinder the sensor's capability to perform real-time monitoring over prolonged periods of time. Therefore, there is ongoing work focused on developing simpler label-free biorecognition schemes that consist of a smaller number of elements while providing the ability to detect targets at the same concentrations as immunoassays. 
The biorecognition scheme explored in this thesis relies on the use of aptamers as biorecognition elements and does not require any kind of tagging for signal amplification. Its application towards the detection of thrombin protein using a fiber transducer is evaluated in Chapter 4.

\subsection{Cellular Analysis}

Emerging trend in the field of biosensing is analysis of intra- and extra-cellular processes. There is a growing demand for cellular analyzers that arises from the increasing importance of tissue engineering field as well as from increasing application of cells in drug discovery and drug screening processes. In both applications there is a need for label-free analytical tools that could be used for monitoring of cellular interaction with their environment and studying of cellular responses to different stimuli. There are different kinds of analytical platforms being developed for analysis and manipulation of cells or cellular 3D structures including electrical, optical and piezoelectrical analyzers. Optical label-free optical platforms successfully applied towards cellular analysis include SPR Biacore ${ }^{2}$ and Corning EPIC devices ${ }^{64,5,6}$. In case of these analyzers cells in order to be studied first have to be adhered to the surface of the optical sensing elements and later studied using these platforms. Due to the size of the commercial analyzers, cellular analysis has to be completed outside cell culture incubators. Handling of cells outside incubators especially over prolonged periods of time can hinder the quality of findings relating to cellular behavior. Thus there is a demand for analytical devices that could be 
integrated into cell culture incubators ${ }^{65}$ and report on cellular behavior while cells remain in their normal environment.

This thesis studies application of the SPR-TFBG sensor as a cellular analytical platform that can be integrated into cell culture incubator allowing for real-time and in situ cellular analysis (Chapter 6) ${ }^{66}$.

\subsection{Thesis Statement and Contribution}

The foundation for this work was established during Yanina Shevchenko's Masters work ${ }^{67}$. The results of Shevchenko Masters's research can be found in Shevchenko and Albert, $(2007)^{45}$ as well as in Y. Shevchenko's Master's thesis (Shevchenko 2007) ${ }^{67}$.

The research presented in Shevchenko's Masters thesis introduced the first version of the SPR-TFBG fiber sensor. The first implementation of the sensor consisted of single-mode fiber (CORNING SMF-28) with $6^{\circ}$ TFBG and a gold coating on the surface of the fiber. Excitation of the plasmonic effect was accomplished through coupling of high order cladding modes into SPR with the help of the TFBG. Both the paper $^{45}$ and the thesis ${ }^{67}$ introduced the concept of the SPR-TFBG sensor and its first implementation. The thesis provided an introduction into characterization of the sensor in terms of its sensitivity towards external refractive index and studied effect of gold thickness of the quality of the SPR coupling. Relatively simple SPR model for planar structures was used to explain observed SPR features in the TFBG spectra. 
The work presented in the $\mathrm{PhD}$ thesis builds upon the previously conducted research and furthers that work by studying the physical principles underlying sensor's behavior, its characterization and its optimization. Furthermore, this thesis also explores how the SPR-TFBG sensor can be applied for real-time and label-free monitoring of several chemical and biochemical processes as well as for monitoring of more complex cellular reactions.

In particular, this thesis explores the following research questions:

\section{Utilization of a model describing excitation of a SRR on the surface of a circular} waveguide. Complicated SPR excitation mechanism involving Bragg grating in a circular waveguide with a metal layer on its surface requires a sophisticated model that could be used to reproduce and confirm experimental findings. Findings presented here were confirmed using FIMMWAVE software that allowed to obtain information about excitation of SPR on a surface of a circular waveguide with a thin metal coating. The FIMMWAVE is a complex fiber mode solver from Photon Design that uses a cylindrical Finite difference algorithm to find all modes that could be supported in a given optical waveguide. The fiber model studied using FIMMWAVE was created to maximally

resemble operation of a real SPR-TFBG sensor ${ }^{48}, 49$. Obtained information revealed that a cylindrical waveguide with a thin gold layer supports a set of cladding modes with a significant imaginary component. It was originally anticipated that plasmon-coupled cladding modes would exhibit strong imaginary components. A non-zero imaginary component is usually characteristic for SPR waves and represents the lossy nature of an electromagnetic wave when coupled with SPR. 
The limitation of this particular approach lays in the fact that the current version of FIMMWAVE cannot be used to simulate excitation of SPR modes on the surface of the metal-coated fiber through TFBG. Effect of TFBG coupling should be found independently and combined with FIMMWAVE findings.

Therefore information about cladding modes including SPR-coupled cladding modes found with the help of FIMMAWAVE was post-processed using additional custom made Matlab code. Post-processing consisted of converting the effective indexes of cladding modes found in FIMMWAVE into wavelengths at which claddings modes could be excited using the TFBG phase-matching condition:

$\beta_{\text {core }}\left(\lambda_{i}\right)+\beta_{c l a d}^{i}\left(\lambda_{i}\right)=\frac{2 \pi \cos (\theta)}{\Lambda}$

where $\theta$ is the tilt angle of the grating, $\Lambda$ is the period of the grating, $\beta_{\text {core }}$ and $\beta_{\text {clad }}$ are propagation constants $\left(\beta=2 \pi \eta_{e f} / \lambda\right)$ of core and cladding modes.

Combining information found using FIMMWAVE and the TFBG phase matching condition (8) allowed for matching of theoretical findings with experimental data. It also allowed for very exact identification of SPR-coupled modes in the TFBG spectra. This step ensured that post-processing and analysis of experimental data were focused on the appropriate SPR modes as illustrated in Shevchenko et al, $2010^{49}$.

2. Data analysis. Although there are several approaches that could be used to interpret spectral response of SPR-TFBG sensor, analysis applied in this work consisted of tracking the amplitude of the most sensitive SPR-coupled resonance. As shown in Chapter 3 relative change of the amplitude can be easily translated into wavelength shift of the SPR position if it is required. 


\section{Polarization selectivity of plasmonic fiber sensor utilizing in-fiber Bragg}

grating: The polarization selectivity of the SPR-coupled cladding modes has been investigated and presented in the Shevchenko et al, (2010) ${ }^{49}$ (Chapter 2). Presented results indicate that the claddings modes coupled to SPR have a strong sensitivity towards the orientation of polarization of applied light. As shown in Chapter 2 a change in the orientation of polarization allows to completely erase the SPR signature from the output spectrum or to maximize the contrast between SPR and non-SPR modes.

4. Sensor's sensitivity and operating range: Performance of the sensor was evaluated in Shevchenko et al, (2010) and Caucheteur et al (2011) ${ }^{51}$. Results presented in Shevchenko et al, (2010) show that the chemical sensitivity of the sensor interrogated by linearly polarized light can be as high as $1370 \mathrm{~nm} / \mathrm{RIU}$. Data analysis based on relative amplitude change approach yielded refractive index uncertainty of $10^{-5}$.

5. Characterization of the polyelectrolyte adsorption: The SPR-TFBG sensor was applied for a real-time characterization of the process of formation of the polyelectrolyte multilayer structure (Shevchenko et al., 2010) ${ }^{48}$ (Chapter 3). Presented results indicate that the sensor can be successfully applied for monitoring of the process of adsorption of polymer monolayers with estimate thickness of each individual monolayer of $1.12 \mathrm{~nm}$. Furthermore, the SPR experimental data was supported by an SPR model describing the excitation of SPR modes on surface of a circular waveguide. It was shown that the combination of experimental SPR data and a SPR theoretical model could be used to evaluate several parameters of the deposited layer such as its thickness or the refractive index (assuming that one of the parameters is known). 


\section{Biosensing and characterization of $K_{d}$ cosntant: The SPR-TFBG sensor was}

applied for the characterization of several biological processes including self-adsorption of DNA on the surface of the of the sensor and detection of target molecules of thrombin ${ }^{30}$. Applied biorecognition scheme employed aptamers as a biorecognition element that were synthesized to bind towards thrombin protein. Explored concentration of target molecules varied from $0.1 \mu M$ to $5 \mu M$. The smallest detectable concentration of thrombin was found to be $22.5 \mathrm{nM}$. It was also shown that a fiber biosensor can be applied towards the evaluation of the dissociation constant $K_{d}$ that is used to characterize affinity between two interacting biomolecules (aptamer and thrombin molecules in this case). $K_{d}$ was found to be $40 \mathrm{nM}$. The capability of the sensor to characterize biomolecules' affinity at such small concentrations using aptamers as label-free receptors illustrates its potential and promise for label-free biosensing applications. Results of this project are presented in the Chapter $4^{30}$.

7. Real-time monitoring of cellular responses: The SPR-TFBG sensor was applied towards motoring of triggered cellular responses. In this project the sensor platform was mounted into a cell culture incubator and was incubated with NIH $-3 \mathrm{~T} 3$ fibroblasts cells for 12 hours. When cells were attached to the surface of the sensor, sensor was used to record triggered cellular responses. Studied cellular processes included cell detachment triggered by trypsin, cellular uptake of serum and cellular death induced by a toxin, sodium azide. Real-time SPR data was complemented by imaging and cellular assays. Use of real-time SPR data together with imaging illustrates that such combination of techniques can be applied to record and interpret early cellular responses that could not 
be monitored using only imaging tools. The results pertaining to this work are presented in the Chapter $5^{66}$.

\subsection{Organization of the Thesis}

This thesis is organized as thesis by article and thus consists of several manuscripts united by a common introduction and a conclusion.

The Chapter 1, which is an introduction, is followed by four chapters which consist of four submitted or already published papers.

Chapter 2 reproduces the article "Polarization-selective grating excitation of plasmons in cylindrical optical fibers" by Shevchenko et al, (2010) that was published in Optics Express ${ }^{49}$. It explores the polarization dependency of SPR excitation using TFBG and studies the chemical sensitivity of the SPR-TFBG sensor.

Chapter 3 reproduces article "In situ monitoring of the formation of nanoscale polyelectrolyte coatings on optical fibers using Surface Plasmon Resonances" by Shevchenko et al, (2010) published in Optics Express ${ }^{48}$. It is focused on the application of the SPR-TFBG sensor towards real-time monitoring of polyelecrolyte monolayer adsorption. Furthermore, the article explores the use of experimental SPR data obtained using a fiber sensor along with the theoretical SPR data obtained using FIMMWAVE software. Analysis based on both experimental and theoretical data allows for extraction of information about several parameters pertaining to the studied process, such as refractive index and thickness of the deposited polyelectrolyte material. Both Chapter 2 
and 3 elaborate on the data analysis method applied towards interpreting experimental SPR data.

Chapter 4 reproduces article "Detection of biological targets and characterization of $K_{d}$ constant" and its supplementary information published in Analytical Chemistry in 2011. In this work the sensor's surface was functionalized with monolayer of aptamers and was subsequently exposed to thrombin solution. The objective of this article was to explore application of given sensor configuration towards biosensing and detection of thrombin target. It also showed that sensor can be successfully applied towards characterization of affinity of two interacting molecules.

Chapter 5 reproduces the article "Surface Plasmon Resonance Fiber Sensor for Real-Time and Label-Free Monitoring of Cellular Behavior" by Shevchenko et al, (2012) that was submitted to Analytical Chemistry Journal (currently under review). The article describes the most recent effort to apply the SPR-TFBG sensor towards monitoring of cellular reactions.

Chapter 6, Summary and Future Work Recommendations outlines major contributions of the presented work, discusses challenges met by the developed fiber biosensor technology and suggests directions for future work. References for Chapters 1 , 5 and 6 are put together provided after Chapter 6. 


\title{
Chapter 2
}

\section{Polarization-selective grating excitation of plasmons in cylindrical optical fibers}

\author{
(published in Optics Letters, 2010) \\ Y. Shevchenko*, C. Chen, M. A. Dakka, and J. Albert \\ Department of Electronics, Carleton University, 1125 Colonel By Drive, Ottawa, \\ ON Canada K1S 5B6 \\ ${ }^{*}$ Corresponding author: yshevch2@connect.carleton.ca
}

\begin{abstract}
We show that the tilted-grating-assisted excitation of surface plasmon polaritons on gold coated single mode optical fibers depends strongly on the state of polarization of the core-guided light, even in fibers with cylindrical symmetry. Rotating the linear polarization of the guided light by 90 degrees relative to the grating tilt plane is sufficient to turn the plasmon resonances on and off with more than $17 \mathrm{~dB}$ of extinction ratio. By monitoring the amplitude changes of selected individual cladding mode resonances we identify a new refractive index measurement method that is shown to be accurate to better than $5 \times 10^{-5}$.

OCIS codes: $240.5440,240.6680,230.1480,060.2310,060.2370$.

C2009 America Institute of Physics.
\end{abstract}


Surface plasmon polaritons (SP) provide an excellent tool for bulk chemical sensing and for monitoring reactions between molecules bound to a sensor's surface and target molecules in solution [1]. The use of core mode gratings to excite SP on metal-coated single mode optical fibers has been proposed before [2-5], but a more recent theoretical calculation has revealed a fundamental problem with this approach [6]: the large optical loss of the SP mode causes a significant broadening and weakening of the grating induced coupling from the core mode to the SP, unless the grating length is kept smaller than the plasmon propagation distance in the metal (a few $\mu \mathrm{m}$ in our case, according to Equation (1) of reference [6]). This effect is due to the fact that the coupling strength of contra-propagating grating couplers increases with grating length. However if the backward reflected mode is lossy the coherent addition of backward waves from each grating plane can only occur over lengths comparable to the mode propagation distance. Therefore the amplitude of the resonant coupling for SP decreases to that of a grating that is only a few $\mu \mathrm{m}$ in length: i.e. weak and very wideband. In the work presented here, we use a tilted fiber Bragg grating (TFBG) to demonstrate experimentally for the first time that grating-coupled resonances do in fact disappear when the grating couples the core mode light to a cladding mode that has a strong SP component. However we also present a technique to determine the peak SPR wavelength as well as sub-nm wavelength shifts of the SPR peak by measuring the amplitude changes of cladding mode resonances provided by the TFBG. This level of sensitivity is critical for detecting small changes associated with the binding of bio-molecules to the metal surface. 
These findings are based on the discovery that the excitation of "SP-active" cladding mode resonances is highly polarization-dependent. Using polarization control, we demonstrate that the TFBG can selectively excite SP-active and non-active cladding mode resonances with up to $17 \mathrm{~dB}$ of differential amplitude at the same spectral location. This observation differs from the results of Allsop et al. [4] who used a similar TFBG but in a D-shaped fiber obtained by lapping one side to within 10 microns of the core. Coating the flat side with metal resulted in the disappearance of the individual cladding mode resonances and broad SP attenuation bands that shifted with changes of the input light polarization azimuth. We use here a gold-coated, otherwise un-modified standard single mode fiber with cylindrical symmetry. We have shown previously that the transmission spectrum of a TFBG in such structures shows anomalous resonances that present strong similarities to SP excitations [7]. However the use of unpolarized light in these earlier experiments prevented the clear identification of plasmon modes.

In our devices (a diagram of the actual configuration can be found in reference [7]), the grating excites a large number of cladding modes [8]. Amongst these modes, only those whose effective index and polarization state are equal or close to the effective index and polarization of a SP can transfer energy to it by tunneling across the metal layer. At wavelengths for which this situation occurs, the amplitude of the resonance decreases sharply because the cladding mode involved is very lossy, as discussed above [6]. The 10 mm-long gratings used for the work reported here were written using the standard process for FBGs (apart from the tilt angle) in hydrogen-loaded standard single mode fiber (CORNING SMF-28) using $248 \mathrm{~nm}$ wavelength excimer laser light and a phase mask. [7-8] The internal tilt of the grating planes, is set at 10 degrees to ensure strong 
coupling to cladding modes with effective indices near 1.3 , suitable for the excitation of plasmons in aqueous environments [8-9]. The sputtered gold coating is deposited in two steps, with the fiber placed horizontally above the sputtering target and rotated by 180 degrees along its axis between each step. So far, we found empirically that the optimal gold thickness for narrow SPR resonances is $50 \mathrm{~nm}$. We used standard instrumentation for the measurement: the un-polarized light from a broadband source $(\mathrm{C}+\mathrm{L}$ band pumped ASE source from JDSU) goes through a polarization controller before going through the fiber sensor and then to an Optical Spectrum Analyzer (Ando AQ6317B). In the polarization controller, we use a linear polarizer (at an arbitrary angle since the input light is perfectly un-polarized) followed by a half-wave plate (HWP) to rotate the linear state of polarization. In the absence of sharp bends in the fiber between the HWP and the sensor, the state of polarization remains linear but arrives at the sensor at an angle that is unknown but that is rotated by a fixed, constant amount relative to the output of the HWP (this assumption is valid for single mode fiber provided the fiber does not move and does not have sharp bends). For testing, the portion of the fiber where the metal-coated grating is located is fixed under slight tension using epoxy adhesive.

Figure 1 presents the transmission spectrum of a typical tilted grating SPR sensor for two orthogonal states of linear polarization. In order to find the appropriate polarization states, the HWP is rotated until the measured transmission spectrum shows the unambiguous SPR signature (top spectrum). In the bottom spectrum, the polarization has been rotated to maximize the amplitude of the cladding mode resonances across the spectrum: no evidence of SP effect shows up in this case (the amount of rotation between the top and bottom spectra is 90 degrees). It is now obvious that the excitation of SP 
depends on the alignment of the linear state of polarization relative to the TFBG tilt direction. Since electromagnetic theory only allows plasmon modes with electric field polarized perpendicular to metal interfaces (radial direction in our case), our results indicate that the TFBG can couple the $\mathrm{LP}_{01}$ core mode of the fiber to cladding modes that have either no radial electric field or a strong radial electric field component at the cladding boundary.

So when the SP-active polarization state is chosen, the effective index of the SPR is found by locating the most attenuated cladding mode resonances. When the external refractive index changes or when the surface of the gold is modified (by the addition of a bio-layer for instance), the complex effective index of the plasmon changes and different cladding modes become attenuated. This is shown in Fig. 2 for immersion in water $\left(n_{D}=1.3324\right)$ and a calibrated refractive index liquid $\left(n_{D}=1.3550\right)$ (supplied by Cargille Corp.). In Figure 2, the locations of the SP maxima are fully supported by simulations carried out using a commercial finite-difference complex mode solver in cylindrical coordinates (FIMMWAVE 5.2, from Photon Design Inc). The simulations provide the complex effective indices (and mode fields) of the structure used in the experiment $\left(\right.$ fiber $\mathrm{n}_{\text {core }}=1.4509, \mathrm{n}_{\text {cladding }}=1.4441$, core and cladding diameter $=8.3 \mu \mathrm{m}$ and $125 \mu \mathrm{m}$ respectively, metal thickness $=50 \mathrm{~nm}$ and complex index $=0.55-\mathrm{i} 11.5$ ). For each mode found, we use the grating phase matching condition (equation (3) of reference [8]) to calculate the resonance wavelength of the mode and we plot the imaginary part of the mode index (the mode loss) as bar graphs on Figure 2.

The simulations indicate that for both values of the external refractive index used in Figure 2 (corrected for dispersion from the $n_{D}$ value, which corresponds to a wavelength 
of $589 \mathrm{~nm}$ ), the loss of a small subset of the cladding modes resonances increases sharply almost exactly at the location of the experimentally observed SP (the small discrepancy is likely caused by uncertainty in the exact value of the permittivity of our sputtered gold layer.

The results of Figure 2 confirm that we can actually measure refractive index changes using SP resonances excited by a grating in a fiber. For "large" refractive index changes such as those in Figure 2, the SPR location can be determined by fitting a suitably shaped envelope function (a second order polynomial over the 4 central resonances for instance). The results of such fits, for experiments carried out in calibrated refractive index liquids, indicate a constant sensitivity of $555 \mathrm{~nm} / \mathrm{RIU}$ (RIU=refractive index unit) with a root mean square (RMS) deviation of 0.00125 in refractive index between the linear fit and actual measurements. The refractive index value of each liquid (i.e. $n_{D}$ ) was measured separately with an Abbe refractometer (with a nominal accuracy of $+/-0.0001$ ). The RMS value provides a good estimate of the uncertainty in the measurement of the refractive indices provided by the device. More importantly however, in most applications of SPR the purpose is not absolute refractometry over large refractive index ranges, but rather the detection of very small changes that are occurring in a highly localized area near the device surface. For these applications a measurement uncertainty of the order of $10^{-3}$ in refractive index is not acceptable, regardless of the $\mathrm{nm} / \mathrm{RIU}$ sensitivity [1]. In order to circumvent this problem, we use the fine spectral comb of cladding mode resonances provided by the TFBG in a slightly different manner. 
Because the slopes of the SPR envelope are relatively large when polarization control is used, typically near $2 \mathrm{~dB} / \mathrm{nm}$, a SPR wavelength shift as small as $50 \mathrm{pm}$ will cause a change of $0.1 \mathrm{~dB}$ in the amplitude of a cladding mode resonance located on the side of the SPR maximum. This is easily measured since only relative measurements are needed: an internal power level and wavelength reference is provided for each grating by the resonance of the core mode reflection (the resonance with the largest wavelength on Figure 1). We demonstrate this high resolution measurement mode by using mixtures of ethanol and water to generate small calibrated index changes in several steps over a total range of $8 \times 10^{-4}$. The measurement error in the mixing ratio was estimated to be 0.001 , yielding a nominal refractive index uncertainty of $2.3 \times 10^{-5}$. Figure 3 shows both the amplitude change of the resonance and the equivalent SPR shift plotted against the refractive index change of the water-ethanol mixture. The results indicate a sensitivity of $1370 \mathrm{~nm} / \mathrm{RIU}$, comparable to the best SPR sensors available, and a RMS refractive index uncertainty of $4 \times 10^{-5}$.

In conclusion, we have shown that the excitation of SP by TFBGs on cylindrical fibers depends strongly on the orientation of the linearly polarized incident core guided light relative to the tilt direction. We also confirmed that a FBG-coupled optical mode that is perfectly phase matched to a SP does not produce a measurable resonance in transmission. However we proposed an accurate method to measure small SP resonance shifts from the changes in the amplitudes of individual cladding mode resonances. This method provides a demonstrated refractive index measurement uncertainty lower than $5 \mathrm{X} 10^{-5}$ over a full range of 0.001 . The device is easier to fabricate, less costly and more 
reliable than other fiber-based SPR sensors because the fiber cladding does not have to be etched, polished, or tapered $[1,4,5]$.

\section{Acknowledgements}

This work is supported by the Natural Sciences and Engineering Research Council of Canada and by LxData Inc

\section{References:}

1. J. Homola, "Surface plasmon resonance sensors for detection of chemical and biological species," Chem. Rev. 108, 462-493 (2008).

2. Y.-J. He, Y.-L. Lo, and J.-F. Huang, "Optical-fiber surface plasmon-resonance sensor employing long-period fiber gratings in multiplexing," J. Opt. Soc. Amer. B, vol. 23, pp. 801-811 (2006).

3. G. Nemova and R. Kashyap, "Fiber-Bragg-grating-assisted surface plasmon-polariton sensor," Opt. Lett. vol. 31, pp. 2118-2120 (2006).

4. T. Allsop, R. Neal,S. Rehman, D. J. Webb, D. Mapps, and I. Bennion, "Characterization of infrared surface plasmon resonances generated from a fiber-optical sensor utilizing tilted Bragg gratings," J. Opt. Soc. Am. B, vol. 25, 481-490 (2008).

5. S. M. Tripathi, A. Kumar, E. Marin, and J.-P. Meunier, "Side-Polished Optical Fiber Grating-Based Refractive Index Sensors Utilizing the Pure Surface Plasmon Polariton," IEEE/OSA J. Lightwave Technol. Vol. 26, 1980-1985 (2008).

6. Y.-C. Lu, W.-P. Huang, and S.-S. Jian, "Influence of Mode Loss on the Feasibility of Grating-Assisted Optical Fiber Surface Plasmon Resonance Refractive Index Sensors," IEEE/OSA J. Lightwave Technol. Vol. 27, 4804-4808 (2009).

7. Y. Y. Shevchenko and J. Albert, "Plasmon resonances in gold-coated tilted fiber Bragg gratings," Opt. Lett. vol. 32, pp. 211-213 (2007). 
8. C. F. Chan, C. Chen, A. Jafari, A. Laronche, D. J. Thomson, and J. Albert, "Optical fiber refractometer using narrowband cladding mode resonance shifts," Appl. Opt. 46, $1142-1149$ (2007).

9. Y. Y. Shevchenko, D.A. D. Blair, M. C. DeRosa and J. Albert, "DNA Target Detection Using Gold-coated Tilted Fiber Bragg Gratings in Aqueous Media," Conference on Lasers and Electro-Optics, 2008, paper CMJ4.

\section{Figure captions}

Fig. 1. Measured transmission spectrum of a tilted FBG with a $50 \mathrm{~nm}$ thick gold coating in water using two orthogonal linear states of polarization (the two spectra are offset by $15 \mathrm{~dB}$ for clarity).

Fig. 2. Measured transmission spectra for immersion of the SPR sensor in two different media. The blue bars correspond to the calculated imaginary part of the effective index for all of the cladding modes simulated for this sensor in a medium of the given refractive index.

Fig. 3. Changes in the amplitude of a single cladding mode resonance adjacent to the plasmon loss peak as a function of the refractive index changes of a water-ethanol mixture. The left vertical scale corresponds to the amplitude change and the right vertical scale to the equivalent SPR shift (obtained by dividing the amplitude change by the slope of the plasmon envelope). The straight line is a best fit to the data points. The inset shows the corresponding transmission spectra of the resonance used. 


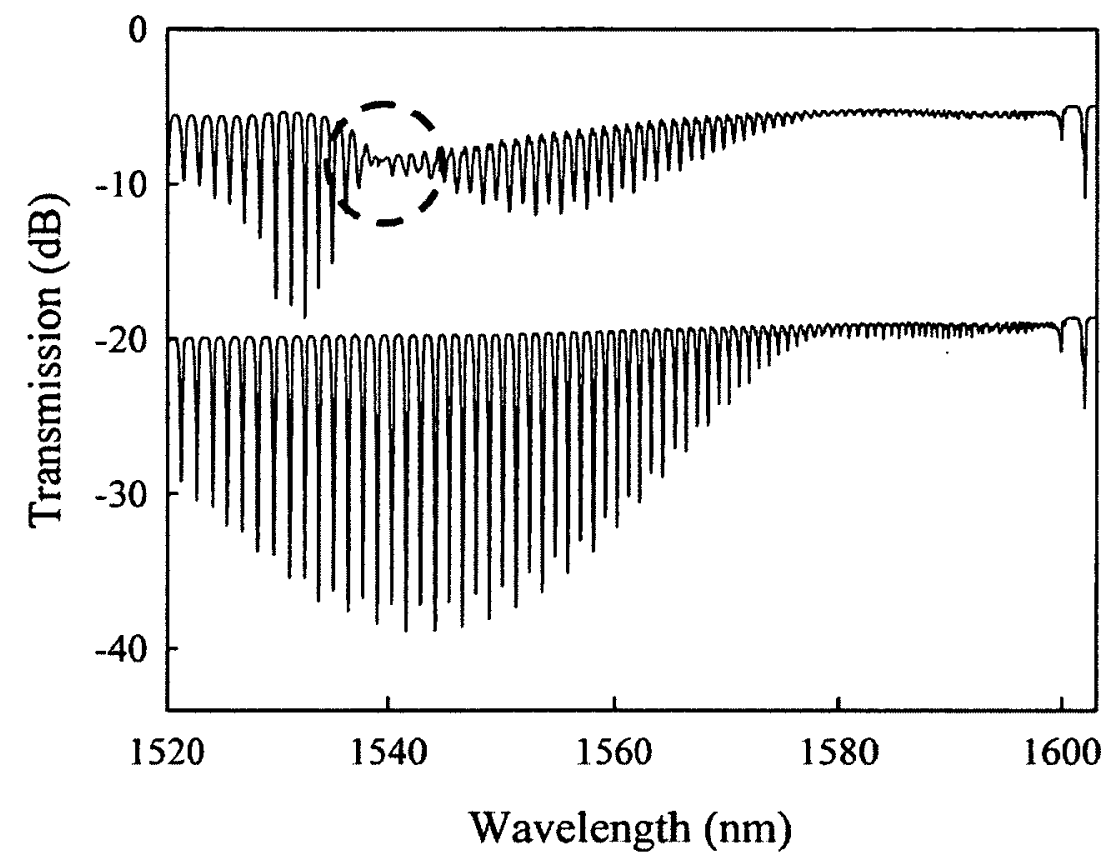

Fig. 1. Measured transmission spectrum of a tilted FBG with a $50 \mathrm{~nm}$ thick gold coating in water using two orthogonal linear states of polarization (the two spectra are offset by $15 \mathrm{~dB}$ for clarity). 


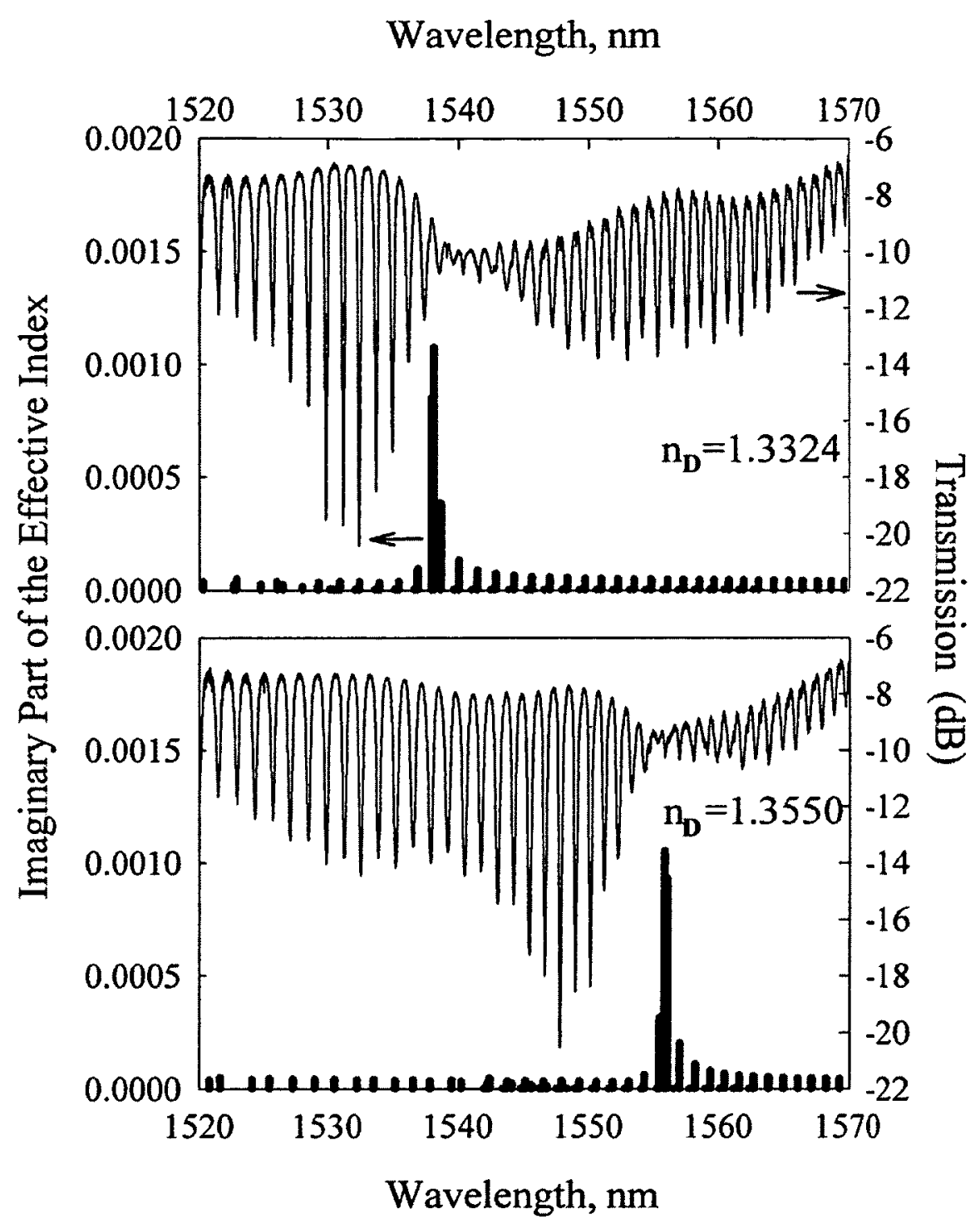

Fig. 2. Measured transmission spectra for immersion of the SPR sensor in two different media. The blue bars correspond to the calculated imaginary part of the effective index for all of the cladding modes simulated for this sensor in a medium of the given refractive index. 


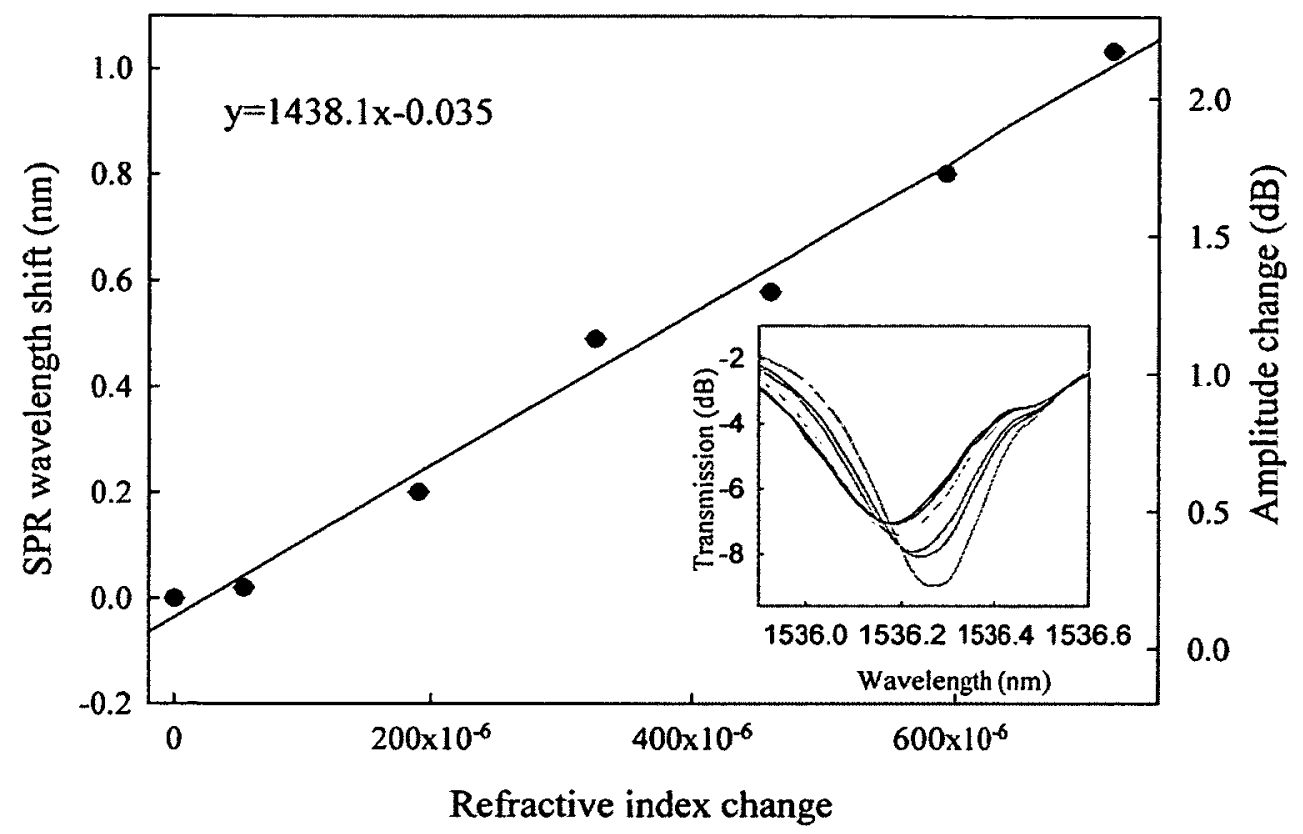

Fig. 3. Changes in the amplitude of a single cladding mode resonance adjacent to the plasmon loss peak as a function of the refractive index changes of a water-ethanol mixture. The left vertical scale corresponds to the amplitude change and the right vertical scale to the equivalent SPR shift (obtained by dividing the amplitude change by the slope of the plasmon envelope). The straight line is a best fit to the data points. The inset shows the corresponding transmission spectra of the resonance used. 


\title{
Chapter 3
}

\section{In situ monitoring of the formation of nanoscale polyelectrolyte}

\section{coatings on optical fibers using Surface Plasmon Resonances}

\author{
(published in Optics Express, 2010) \\ Yanina Shevchenko, ${ }^{1, *}$ Nur Uddin Ahamad, ${ }^{2}$ Anatoli Ianoul ${ }^{2}$ \\ and Jacques Albert ${ }^{1}$ \\ 'Department of Electronics, Carleton University, 1125 Colonel By Drive, Ottawa, ON, K1S5B6, Canada \\ ${ }^{2}$ Department of Chemistry, Carleton University, 1125 Colonel By Drive, Ottawa, ON, KIS5B6, Canada \\ -yshevch2@connect.carleton.ca
}

Abstract: Deposition of a conformal nanoscale polymer coating was characterized using

a fiber SPR sensor. The sensor platform consisted of an unmodified gold-coated single

mode fiber where SPR was excited through the coupling of the core mode into the cladding modes using a Tilted Fiber Bragg Grating. The results from this study show how the sensor can monitor in real time the formation of polyelectrolyte coatings during a process consisting of several stages of immersion. The experimental data was further calibrated by simulations and Atomic Force Microscope imaging allowing us to determine the thickness and refractive index of the adsorbed polyelectrolyte.

C2010 Optical Society of America

OCIS codes: (60.2370) Fiber optics sensors; (60.3735) Fiber Bragg gratings; (240.6680) Surface Plasmons.

\section{References and links}

1. Y. Y. Shevchenko, and J. Albert, "Plasmon resonances in gold-coated tilted fiber Bragg gratings," Opt. Lett. $32,211-213(2007)$.

2. Y. Y. Shevchenko, D. A. D. Blair, M. C. DeRosa, and J. Albert, "DNA Target Detection Using Gold-coated Tilted Fiber Bragg Gratings in Aqueous Media," in Conference on Lasers and Electro-Optics, (Optical Society of America, 2008). 
3. C. F. Chan, C. Chen, A. Jafari, A. Laronche, D. J. Thomson, and J. Albert, "Optical fiber refractometer using narrowband cladding mode resonance shifts," Appl. Optics 46, 1142-1149 (2007).

4. L. Shao, Y. Shevchenko, and J. Albert, "Intrinsic temperature sensitivity of tilted fiber Bragg grating based surface plasmon resonance sensors," Opt. Express 18 (11), 11464-11471 (2010).

5. Y. Shevchenko, C. Chen, M. A. Dakka, and J. Albert, "Polarization-selective grating excitation of plasmons in cylindrical optical fibers," Opt. Letters 35 (5), 637-639 (2010).

6. B. Spackova, M. Piliarik, P. Kvasnicka, C. Themistos, M. Rajarajan, and J. Homola, "Novel concept of multi-channel fiber optic surface plasmon resonance sensor," Sensors Actuat. B-Chem. 139, 199-203 (2009).

7. G. Decher, J. D. Hong, and J. Schmitt, "Buildup of ultrathin multilayer films by a self-assembly process: III. Consecutively alternating adsorption of anionic and cationic polyelectrolytes on charged surfaces," Thin Solid Films 210-211, 831-835 (1992).

8. H. Jeong, W. Pyun, S. Y. Yang, "Gold nanoparticle-hybridized "Nano-sponge" polymer coatings to enhance the reliability and sensitivity of biosensors", Macromol. Rapid Comm. 30 (13), 1109-1115 (2009).

9. Y. Da, C. Chen, B. Li, M. Zhou, E. Wang, and S. Dong, "Layer-by layer electrochemical biosensor with aptamer-appended active polyelectrolyte multilayer for sensitive protein determination," Biosens. Bioelectron. 25, 1902-1907 (2010).

10. Y. Sun, D. Song, Y. Bai, L.Wang, Y. Tian, and H. Zhang, "Improvement of surface plasmon resonance biosensor with magnetic beads via assembled polyelectrolyte layers," Anal. Chim. Acta 624, 294-300 (2008).

11. D. W. Kim, Y. Zhang, K. L. Cooper, and A. Wang, "Fibre-optic interferometric immuno-sensor using long period grating," Electron. Lett. 42, 324-325 (2006).

12. F. J. Arregui, 1. R. Matias, Y. Liu, R. O. Claus, "Optical fiber humidity sensor using a nano Fabry-Perot cavity formed by the ionic self-assembly method," Sensors Actuat. B-Chem. 59 (1) 54-59 (1999).

13. S. T Dubas, and J. B. Schlenoff, "Factors controlling the growth of polyelectrolyte multilayers," Macromolecules 32 (24), 8153-8160 (1999).

14. Y. Fu, H. Xu, S. Bai, D. Qiu, J. Sun, Z. Wang, and X. Zhang, "Fabrication of a stable polyelectrolyte/Au nanoparticles multilayer film," Macromol. Rapid Comm. 23, 256-259 (2002).

15. F. Caruso, D. N. Furlong, K. Ariga, 1. Ichinose, and T. Kunitake, "Characterization of polyelectrolyte-protein multilayer films by atomic force microscopy, scanning electron microscopy, and fourier transform infrared reflection-absorption spectroscopy," Langmuir 14, 4559-4565 (1998).

16. D. Briggs, Surface Analysis of Polymers by XPS and Static SIMS (Cambridge, 1998). 
17. H. Raether, Surface Plasmons on Smooth and Rough Surfaces and on Gratings (Springer -Verlag, 1988).

18. R. C. Jorgenson, and S. S. Yee, "A fiber-optic chemical sensor based on surface plasmon resonance," Sensors Actuat. B-Chem. 8, 213-220 (1993).

19. G. Nemova, and R. Kashyap, "Novel fiber Bragg grating assisted plasmon-polariton for bio-medical refractive-index sensors," J. Mater. Sci-Mater. El. 18, 327-330 (2007).

20. A. J. C. Tubb, F. P. Payne, R. Millington, and C. R. Lowe, "Single mode optical fibre surface plasma wave chemical sensor," Electron. Lett. 37, 1-5 (1995).

21. R. Alonso, F. Villuendas, J. Tornos, and J. Pelayo, "New 'in-line' optical-fibre sensor based on surface plasmon excitation," Sens. Actuators A Phys. 37, 187-192 (1993).

22. G. Laffont, and P. Ferdinand, "Tilted short-period fiber-Bragg-grating-induced coupling to cladding modes for accurate refractometry," Meas. Sci. Technol. 12, 765-770 (2001).

23. A. lanoul, and A. Bergeron, "Spatially inhomogeneous enhancement of fluorescence by a monolayer of silver nanoparticles," Langmuir 22, 10217-10222 (2006).

24. M. Schönhoff, "Layered polyelectrolyte complexes: physics of formation and molecular properties," J. Phys.: Condens. Matter 15, R1781-R1808 (2003).

25. J.A. Jaber, and J.B. Schlenoff, Counterions and water in polyelectrolyte multilayers: a tale of two polyions, Langmuir 23, 896-901 (2007).

26. I. H. Malitson, "Interspecimen comparison of the refractive index of fused silica," J. Opt. Soc. Am. 55, 1205$1209(1965)$

27. P. B. Johnson, and R. W. Christy, "Optical constants of the noble metals," Phys. Rev. B 15, $4370-4379$ (1972).

28. V. M. Zolotarev, B.A. Mikhailov, L. I. Alperovich, and S.I. Popova, "Dispersion and absorption of liquid water in infra-red and radio-frequency regions," Opt. Comm. 1 (6), 301-302 (1970)

29. S. T. Dubas, and J. B. Schlenoff, "Swelling and smoothing of polyelectrolyte multilayers by salt," Langmuir $17,7725-7727(2001)$

1. Introduction: In 2007, we proposed and demonstrated the first Surface Plasmon Resonance (SPR) fiber sensor implemented using a conventional unmodified single mode fiber with a tilted Bragg grating inscribed in its core [1]. Overall, SPR sensors can be 
used to measure refractive indices, thickness changes and the occurrence of chemical reactions or biomolecular recognition events over nanometer-scale thicknesses. SPR sensors implemented in optical fibers are small, can be used remotely, and are relatively easy to fabricate. The operating range of the tilted fiber Bragg grating-SPR (TFBG-SPR) sensor, in terms of the refractive index of the material in which it is embedded, is determined by the tilt angle of the grating. It is therefore possible to tailor the design of the sensor for applications in various media. As shown by our group earlier $[2,3]$ a tilt angle of 10 degrees optimizes the response of the sensor for measurements made in water and other aqueous environments. In addition to its broad operating range, the sensor has an inherently small cross-sensitivity to temperature that is further simplified by the fact that one of the optical transmission features (the core mode back reflection upon itself) can be used to measure the temperature at the device in real time without interference from changes in the outer medium [3,4]. It was also demonstrated recently [5] that polarization control of the light launched into the fiber core leads to a significant improvement of the sensor response by clearly separating SPR-active and non-active cladding modes. The concept of a Bragg grating assisted SPR sensor in a conventional fiber has also been studied theoretically in great detail and showed to be able to compete with bulkier and costlier SPR devices [6].

In this paper, we demonstrate how a TFBG-SPR sensor can be used for in situ and real time detection of individual nanometer-thick polyelectrolyte layers formed during a Layer-by-Layer self-assembly process [7]. Polyelectrolytes belong to a broad class of polymer molecules that carry electrical charges that can interact with other charged molecules. Given their ability to assemble on charged surfaces and to form multilayer 
structures on substrates of almost completely arbitrary shapes, polyelectrolytes have found important uses in many fields, including chemical sensing and biosensing [8, 9]. In such applications, polyelectrolyte films can be used as a coating material and substrate for hosting chemical reactions $[10,11]$, or they can be used as the sensing material itself [12]. In the work presented here, the formation of a polyelectrolyte multilayer structure was studied using alternating immersion of a gold coated TFBG-SPR optical fiber into two well known polyelectrolytes: negatively charged poly (sodium-4-styrenesulfonate) (PSS) and positively charged poly (diallyldimethylammonium chloride) (PDADMAC). Readings from the TFBG-SPR sensor make it possible to see the dynamics of the polyelectrolyte multilayer formation (build-up) through the immersion stages in various solutions. The monitoring and detection of the formation of multilayered polyelectrolyte films in situ and in real time by the TFBG-SPR sensor has several important consequences: 1) the sensing device can be used as a process monitor for the deposition on other substrates in mass production environments; 2) it is possible to fabricate fiberoptic biochemical sensors based on the polyelectrolyte-coated, gold-coated fiber grating devices with very precise layer compositions and thicknesses; and 3) the sensor can be used for the characterization of other properties of the deposited films besides thickness, such as refractive index or density (with help from complementary measurements, as shown below). These features of our TFBG-SPR sensor present a great advantage since it is generally difficult to characterize the adsorption of nanoscale coatings on non-planar surfaces using the most common thin film characterization methods such as UV-visible absorption spectroscopy [13], ellipsometry, Atomic Force Microscope (AFM) [14], Scanning Electron Microscopy (SEM) [15], or X-ray photoelectron spectroscopy [16]. 
This is even more difficult when the chemical reactions occur inside process chambers filled with liquids (such as those required for the LbL process).

Our results are presented as follows: the TFBG-SPR platform is briefly described and its optical properties discussed in the context of the detection of conformal thin films on the outer surface of the fiber; then the fabrication of the device and measurement techniques are presented followed by the description of the LbL process and of the polyelectrolyte solutions. Experimental results for two tests are presented and discussed, with help from simulations of the coated TFBG-SPR structures. We show that it is possible to calibrate the optical response of the device to a particular process and choice of polyelectrolytes by a combination of modelling and post-fabrication final thickness measurement using AFM. It is important to note that once this calibration is made, there is no further need for AFM measurements to extract the thickness data from subsequent optical measurements with the SPR-TFBG sensor.

\section{Description and implementation of the TFBG-SPR Platform}

Our sensor platform utilizes the SPR effect. One of the key requirements of any optical SPR configuration is the presence of an interface between metal and dielectric materials that have optical properties suitable for the excitation of Surface Plasmon Waves (SPW) [17]. For light to excite a SPW at that interface, the SPR phase matching condition must be satisfied: the longitudinal component of the propagation constant $\beta_{\text {inc }}$ of the light wave used to excite the SPW must be equal to the propagation constant $\beta_{\mathrm{p}}$ of the SPW that can be supported by these materials. $\beta_{\mathrm{p}}$ is calculated as follows: 


$$
\beta_{p}=\frac{\omega}{c} \sqrt{\frac{\varepsilon_{e x} \xi_{m}}{\left(\varepsilon_{e x t}+\varepsilon_{m}\right)}}
$$

where $c$ is the speed of light in vacuum, $\omega$ is the angular frequency of the light, while $\varepsilon_{m}$ and $\varepsilon_{\text {ext }}$ are the relative permittivity of the metal and of the material that is adjacent to the metal interface where the SPW is located (the outside surface of the metal coating in our case).

In order for the SPW to be confined to the surface without radiating into the surrounding medium, the magnitude of $\beta_{\mathrm{p}}$ must be larger than the magnitude of the propagation constant of the light waves in that medium $\left(\beta_{\text {ext }}\right)$. As a result it is impossible to excite a SPW with a light wave incident from above or below the metal film unless some phase matching or other techniques are used. The usual solution for uniform structures is to use tunneling of the light from a high index medium (i.e. large $\beta_{\text {inc }}$ ), across the metal to its outer surface where the following condition can be met for the SPW to be excited:

$$
\beta_{p}=\beta_{i n c}>\beta_{e x t},
$$

The most widespread implementation of this configuration, the Kretschmann-Raether configuration, makes use of a high index glass prism with a metal-coated base [17]. For media with refractive indices lower than that of the prism, light beams incident on the base of the prism can be totally reflected (for a range of angles that depends on wavelength and the refractive indices of the media) and the associated evanescent field can be used to tunnel across the metal and couple to a SPW, provided the metal layer is not too thick. 
Achieving similar conditions in glass optical fibers is possible because when the external medium has a refractive index lower than that of the glass (near 1.45 in the near infrared for silica fibers), light again is confined in the glass by total internal reflection. The challenge for fiber-based SPR sensors is to find a mechanism which would allow coupling of light propagating in the core or cladding to the SPW, and more importantly, which would provide precise control over this coupling. As noted in the Introduction, we have found a way to do this in conventional single mode fibers [1], [5], and [4] without having to use multimode fibers [18], custom-designed fibers [19], or fiber structure modifications $[20,21]$.

Figure 1 presents a schematic diagram that highlights the analogy between the most common bulk optic SPR platform (the Kretschmann-Raether configuration that uses angle or wavelength scanning to tune $\left.\beta_{\text {inc }}[17]\right)$, and the wavelength selective excitation of individual cladding modes by a TFBG. In this configuration, we can individually and separately excite over one hundred cladding modes just by changing the wavelength of the core guided light, making it very easy to find modes that are phase matched to a wide range of SPR conditions. Once a SPR has been identified in the fiber transmission spectrum, the differential response of the cladding mode resonances that are phase matched to the SPW can be used to monitor very precisely any changes of the SPR conditions. In particular, we can measure changes induced by thickness or refractive index modifications in the material that is immediately adjacent to the metal coating, within the evanescent tail of the plasmon wave field. Since the structural integrity of the fiber is not compromised in this configuration, the sensor structure is reliable and very easy to mass-produce at low cost. 


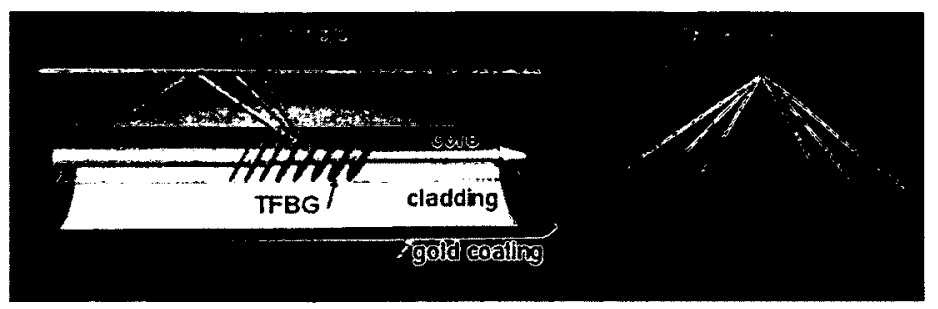

Fig. 1. Analogy between the TFBG and prism-based excitation of plasmon waves by resonant tunneling across a metal layer from a material with a refractive index higher than the surrounding medium.

The main characteristic of the TFBG is that planes of raised refractive index are tilted away from the perpendicular to the fiber's longitudinal axis. Gratings with relatively small tilt angles $\left(2^{\circ}-10^{\circ}\right)$ allow the excitation of several tens of cladding modes $[3,22]$ as shown in Fig. 2. Each of the excited modes is guided by the cladding with effective indices ranging from less than 1.300 to approximately 1.445 (for the resonances shown). When this fiber is coated with a thin metal layer, this range of effective indices corresponds to the possible plasmon effective indices that the grating can couple to.

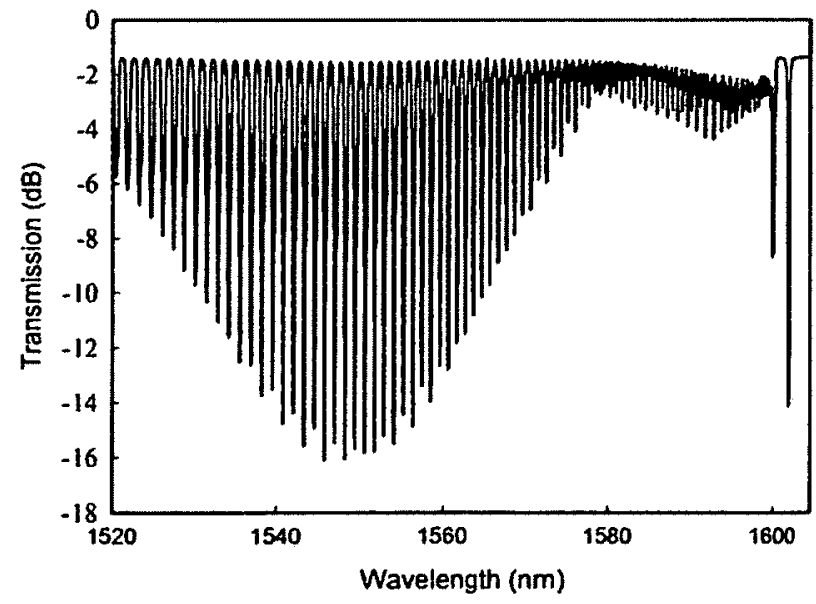

Fig.2. Transmission spectrum of a bare TFBG with $10^{\circ}$ of tilt measured in air. 
The propagation constant of each cladding mode in the spectrum of Fig. 2 can be found from the phase matching condition between the core mode, each cladding mode, and the grating period of the TFBG:

$$
\beta_{\text {core }}\left(\lambda_{i}\right)+\beta_{\text {cldd }}^{i}\left(\lambda_{i}\right)=\frac{2 \pi \cos (\theta)}{\Lambda},
$$

where $\theta$ is the tilt angle of the grating, $\Lambda$ is the period of the grating, and each

propagation constant $\left(\beta=2 \pi n_{e f} / \lambda\right)$ must be evaluated at the wavelength at which the coupling occurs for the given cladding mode [22]. In particular, the dispersion of the fiber refractive indices with wavelength must be taken into account in order to reproduce the spectrum of Fig. 2.

The combination of Equations 1 to 3 makes it possible to determine which cladding mode can be used to excite SPR for a given set of grating parameters and refractive indices of the materials used. Therefore, as shown in [5], by monitoring the SPR wavelength shift (through the shifts in the amplitudes and wavelengths of the cladding modes which are coupled to the SPR) we can extract very precise information related to the change of the bulk refractive index of the dielectric in proximity to the surface of the metal. We show here that nm-scale thin films can also be measured accurately.

\section{Experimental Methods}

\subsection{Fabrication of the Sensor}

The fiber used to fabricate the sensors was a standard single-mode telecommunication fiber (Corning SMF-28) that was saturated with hydrogen prior to writing the grating; the 
fiber was kept for 14 days at room temperature in a pressure vessel maintained at a pressure of 140 atm to increase its photosensitivity to the ultraviolet light used in the process of grating writing.

The $1 \mathrm{~cm}$-long tilted gratings were written in the fiber core using the phase mask technique with excimer laser irradiation at $248 \mathrm{~nm}$. To obtain a "tilted" grating, we rotated the fiber and phase mask together around a vertical axis that was perpendicular to the fiber. The tilt angle of the grating determined the wavelength separation between the core mode (Bragg) resonance and the peak of the envelope of the cladding mode resonances in transmission [3]. The tested gratings had a $10^{\circ}$ tilt with a Bragg resonance at $1601 \mathrm{~nm}$. As shown in Figure 1, this choice of grating parameters resulted in a relatively wide cladding mode spectrum (over $100 \mathrm{~nm}$ ), centered near $1550 \mathrm{~nm}$, which allowed the sensor to work in various aqueous solutions with refractive indices around 1.31-1.34 (we need cladding modes with effective indices slightly higher than the outside medium index to couple to SPR). All gratings were subjected to a thermal stabilization process that involved a quick anneal (approximately 1 minute at $400^{\circ} \mathrm{C}$ ) to remove the unstable portion of the ultraviolet induced refractive index change and a 12 hour annealing at $120^{\circ} \mathrm{C}$ to outgas all remaining hydrogen.

The final step in the preparation of the sensor involved the deposition of a gold layer on the surface of the fiber where the grating was located. The gold coating was deposited using a table-top vacuum sputtering system with the fiber axis parallel to the target. To render the coating relatively uniform around the circumference, two depositions were carried out with a fiber rotation of 180 degrees between depositions. For the two tests reported here, we used nominal gold film thicknesses of 20 and $50 \mathrm{~nm}$ respectively, as 
determined by the thickness measured on a flat control sample in the same sputtering system.

\subsection{Measurement setup}

The optical transmission of the sensors was carried out using two different kinds of instruments. For the first set of measurements, an Optical Sensing Analyzer (model Si720 from Micron Optics) was used in combination with a Polarization Controller from JDS Uniphase and the measurement resolution was set to $0.0025 \mathrm{~nm}$. The polarization controller was used to optimize the linear polarization of the light incident on the grating [5]. This system allowed the interrogation of the sensor at a rate of 1.2 seconds per full spectrum measurement. In the second case, an un-polarized broadband amplified spontaneous emission source (JDS Uniphase) and an optical spectrum analyzer (ANDO AD6317B) with the resolution set at $0.015 \mathrm{~nm}$ were used to interrogate the sensor over long periods (several hours) at a low recording rate of around 1.5 minutes per full spectrum measurement. The use of unpolarized light decreased the sharpness of the SPR response (since only half the light was in the correct polarization to excite the plasmon wave on the metal), but still allowed accurate measurements to be made. However, the measurements obtained with the fast sweeping source are slightly noisier.

During the experiments, the sensors were fixed by a UV-sensitive adhesive in a 7.5 by $2 \mathrm{~cm}$ plastic cell provided with a carved out indentation $(45 \mathrm{~mm}$ by $5 \mathrm{~mm}$ ) which was filled with the various solutions during the tests. A glass slide was placed on top of the fluid-filled cell during each measurement step to ensure that evaporation did not occur: without this precaution the sensor would pick up the refractive index changes caused by evaporation in the small volume of the cell. All experiments were conducted at a constant 
temperature of $23^{\circ} \mathrm{C}$. In principle this does not matter since the TFBG itself is inherently temperature-insensitive [3], and the intrinsic temperature sensitivity of the TFBG-SPR effect has been found earlier [4] to be negligible relative to the shifts that were observed here.

\subsection{Layer-by-Layer deposition method}

The two oppositely charged electrolyte solutions used in this study were prepared from a poly (diallyldimethylammonium chloride) solution (PDADMAC; $35 \mathrm{wt} \%$ in water, MW $<100,000$ ), and poly (sodium- 4-styrenesulfonate) (PSS; MW 70,000), both from Aldrich

Inc. A stock solution of $10^{-3} \mathrm{M} \mathrm{NaCl}$ was prepared using $18.2 \mathrm{M} \Omega \mathrm{cm}$ de-ionized water as a solvent. This solution was used for making $1 \mathrm{mg} / \mathrm{ml}$ of PSS and PDADMAC solutions.

Before the experiment, the gold-coated sensors were cleaned with ethanol and 18.2 $\mathrm{M} \Omega \mathrm{cm}$ de-ionized water. To deposit the polymers on the surface of the sensors, the sensors were first fixed in the cell. Second, the cell was filled with $800 \mu \mathrm{L}$ of PDADMAC solution for 10 minutes; this was followed by a wash with de-ionized water (the washing step lasting approximately 4 minutes) and then the fiber was left to dry in air. The same process was repeated with the PSS solution. These four steps constitute the formation of one PDADMAC-PSS bilayer consisting of pair of PDADMAC and PSS monolayers. The four-step procedure was repeated four times during the first test in order to deposit 4 bilayers and 15 times during second test in order to deposit 15 bilayers. 
SPR measurements were recorded during the sensor immersions in the polyelectrolyte solutions, and also during the immersions in de-ionized water that took place in between the polymer depositions.

\section{Experimental Results}

\subsection{Optical transmission measurement}

A typical transmission spectrum of the sensor immersed in the polymer solution is presented in Fig. 3. The circled region on the spectrum shows which cladding modes tunnel across the gold film [5], thereby incurring loss and decreasing the amplitude of the corresponding resonances (see Fig. 1 for a comparison with a transmission spectrum with no SPR).

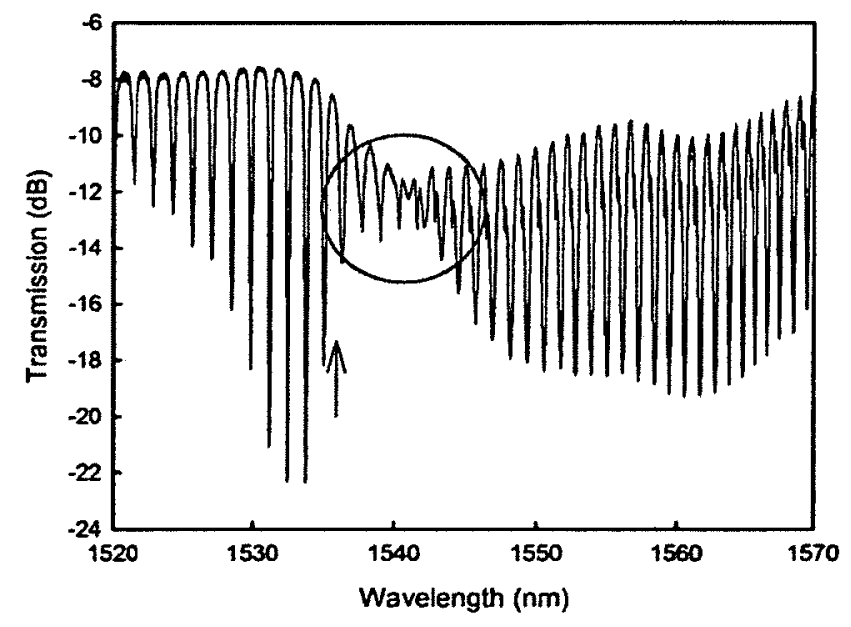

Fig. 3. Transmission spectrum of a TFBG-SPR sensor interrogated using polarized light and immersed in the polyelectrolyte solution. The location of the SPR-coupled set of cladding modes is shown with the circle. The arrow points at the cladding modes whose amplitudes are used to infer the SPR shift (see text for details). 
Any change in the refractive index of the material within the evanescent tail of the plasmon wave will cause a change of the effective index of the surface plasmon mode (as seen from Eq. 1), and therefore a wavelength shift in the position of SPR. Position of the centre of SPR region could possibly be found as the centre of the envelope which could be used to fit top or bottom of the resonances shown with the red circle in Fig.3. Since the wavelength shifts resulting from the deposition of monolayers of polyelectrolytes are expected to be much smaller than the spectral width of the SPR, it is expected that the traditional way to track SPR through fitting might result in a low signal to noise ratio. Therefore, as explained in more detail in [5], we measured the amplitude change of several individual cladding mode resonances located on the edge of the SPR (the most sensitive resonance is the one shown with an arrow in Fig. 3) instead of trying to fit the SPR envelope at each step. The SPR wavelength shift in $\mathrm{nm}$ was then calculated by dividing the amplitude change of the resonance in $\mathrm{dB}$ by the local slope of the SPR envelope $(\mathrm{dB} / \mathrm{nm})$.

\subsection{SPR Film growth measurement and interpretation of the experimental results}

Results of the two experiments, corresponding with the deposition of 4 and 15 bilayer structures are shown in Figs. 4 and 5. The main idea behind conducting two tests was to not only show the reproducibility, but also to explore the real-time dynamics of the polyelectrolyte coating formation over short and long time scales (thin and thick final layers).

During the first test, data was acquired every 1.2 seconds, providing an insight into the formation of the polyelectrolyte coating from the first second of the first immersion. 
The sensor response during immersions in the polymer solutions, each lasting 10 minutes, was combined with responses measured in water (the drying steps cannot be shown because the SPR effective index for air as the outer medium lies outside the range of effective indices accessible with our device). Each immersion in water lasted around 1 minute and can be recognized in Fig. 4 by a strong downward SPR signal change due to the refractive index mismatch between polymer solutions and water. The inset displayed in Fig.4 shows a combination of the sensor responses measured only in water (data related to soaking in polymer solutions was eliminated).

Overall, Fig. 4 shows a step-by-step positive SPR change following the deposition of each bi-layer. However a closer look at the SPR shifts during the deposition of bi-layers reveals the surprising result that the shift is essentially inexistent during the immersions in the two polymer solutions, but jumps sharply following the rinsing and drying steps, especially after the immersion in the second polyelectrolyte. The inset of Fig. 4 further shows definite positive SPR shifts during the short rinsing stages.

The results of the second experiment where a "thicker" 15-bilayer structure was deposited are shown in Fig. 5. Several measurements were taken during each of the 10min immersions in the polymer solutions. SPR dynamics during the rinsing steps has been removed from the figure to highlight the growth of the film during each immersion. The results of this second test confirm those of the first and indicate a monotonic increase of the SPR wavelength as a result of the deposition of the first 22 monolayers. This increase is very regular except for the first few layers, where the polymer film is beginning to form, and the last few layers where the growth is more inconsistent. As 
discovered in the first test, here SPR growth mostly stems from intermediate steps consisting of rinsing in water and drying in the air.

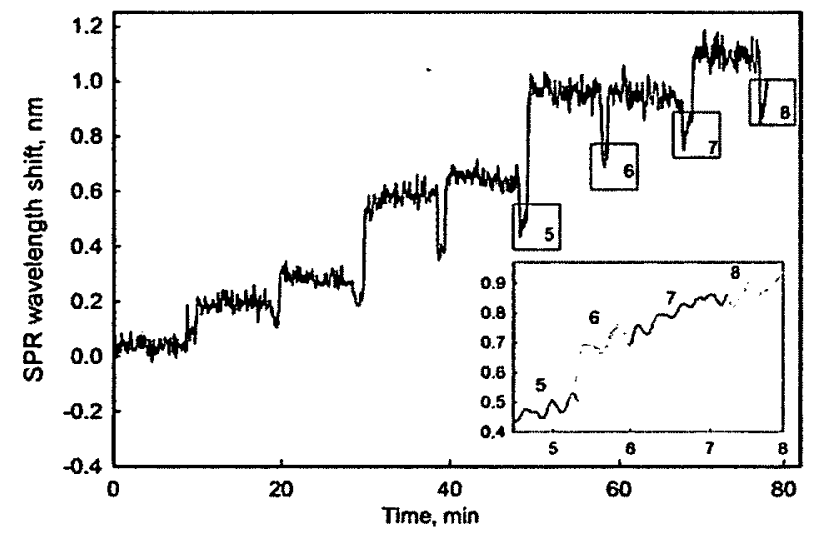

Fig. 4. Change of the SPR wavelength observed during the immersions in the polymer solutions and during soaking in water. Each $10-\mathrm{min}$ immersion in the polymer solution is followed by a 1-min immersion in deionized water. Inset shows SPR change observed in water. Shown data was smoothed using moving average filter.

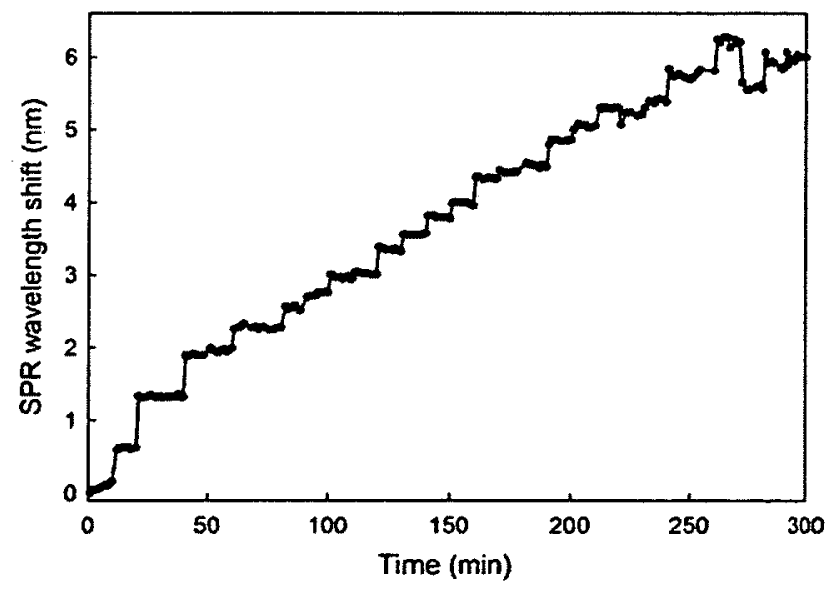

Fig. 5. The SPR wavelength change during the deposition of a thicker polymer film (measurements acquired only in the polymer solutions). 


\subsection{Experimental verification of the film deposition}

An AFM scan of the sensor surface before and after the deposition of the 15 bi-layers was conducted to corroborate the SPR measurements. The scanning was performed in semicontact mode and the scan of the film was completed several days after the deposition when the polymer coating had completely dried out.

Images (a) and (b) in Fig. 6 show the surface of the sensor before and after the deposition of a polymer film while Fig. 7 shows the height profile of the surface of the polyelectrolyte coating across the boundary of a scratch made on the coating. The scratch was made mechanically and was introduced in order to determine the thickness of the deposited structure. The total thickness of the deposited layers (gold plus polymer) was found to vary between 22 and $60 \mathrm{~nm}$ with an average thickness of $36.8 \mathrm{~nm}$. Removing the gold thickness from this measurement $(20 \mathrm{~nm})$, we obtained an average value of $16.8 \mathrm{~nm}$ for the polymer layer, i.e. $1.12 \mathrm{~nm}$ per bi-layer, consistent with values reported for this type of deposition [23]. As expected, there is a large variance in the total thickness (easily observed in Fig. 6(b)) as the deposition appears non-uniform.
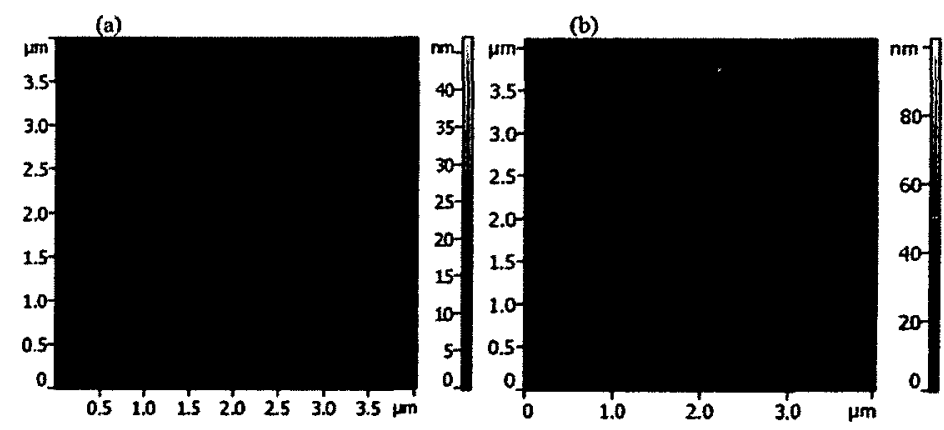

Fig. 6. (a) AFM image of the gold coating before deposition of the polyelectrolyte film.

(b) AFM image of the gold coating with 30 monolayers of the polyelectrolytes. The image was taken near the boundary of a scratch which was done to remove the polymer coating in order to determine its thickness. 


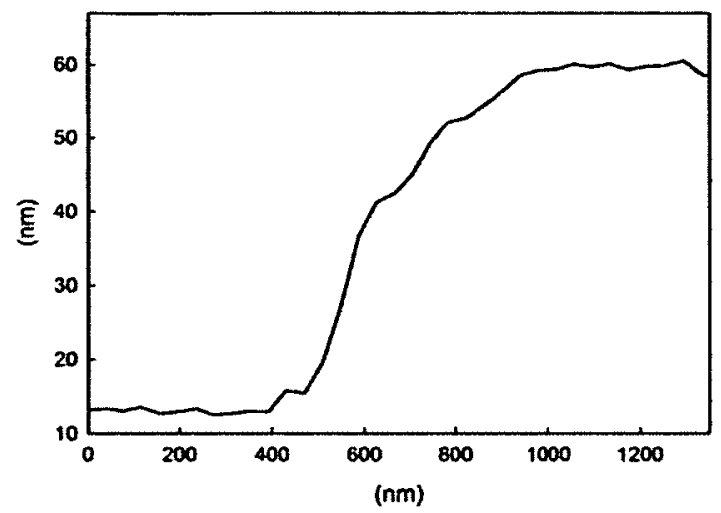

Fig. 7. One of the measured height profiles of the coating across the boundary of the scratch on the surface of the fiber.

\section{Data analysis and discussion}

\subsection{Layer build-up dynamics}

For the high temporal resolution measurement reported in Fig. 4, the most striking feature is that the bi-layer organization appears rather unexpectedly to occur mostly during rinsing and drying stages instead of during soaking in the polyelectrolytes. The negative SPR jump observed during water rinsing is caused by the lower refractive index of the water relative to the buffer solutions that were used for the polyelectrolyte deposition. But the gradual positive SPR shifts observed during the rinsing stages also indicate that the films become optically denser. These shifts do not converge to a steady value however and this may be an indication that the process had not stabilized and that different layer densities might have occurred if longer rinsing durations had been used.

The fact that the real-time data recorded during the adsorption of the polyelectrolyte molecules does not show a significant SPR shift, while soaking in water and drying steps always results in a SPR increase, may be explained by the ionic strength of the solutions 
used and its impact on the configuration of the polyelectrolyte molecules. More specifically, polyelectrolyte chains in an aqueous solution of ionic salt (1 $\mathrm{mM} \mathrm{NaCl})$, assume a coiled configuration due to the screening of charge along the polymer chain by counter ions in the solution. Adsorption of polyelectrolytes with such coiled configuration to the surface of the sensor results in a layer with a certain thickness and density that is strongly related to the amount of coiling [24]. However, when the sensor with the adsorbed polyelectrolyte molecules is immersed in deionized water, most of the counter ions are expelled from the polymer matrix [25]. Then the charge density in the polymer chains increases and in turn stresses the polymer backbone into a flatter configuration, making the film denser. This process repeats for each group of deposited bi-layers.

The longer term measurements reveal the same kind of build-up with distinct SPR shifts occurring mostly right after the rinsing step of each bi-layer, but also that the net shift following each bi-layer is not uniform. We believe this to be due to the fact that the layers themselves are not uniform, as shown by the subsequent AFM images of the final coatings. These observations indicate the potential of our TFBG-SPR as a real-time process monitoring tool for nanoscale deposition processes in liquids.

\subsection{Extracting additional information from the $S P R$ shift data}

The shift of the SPR resonance arising from the deposition of a thin coating on the surface of the fiber depends on the thickness of the added layer but also obviously on its refractive index. Numerical simulations were performed to confirm the experimental results obtained from the sensor and to evaluate the refractive index of the deposited 
polymer film. The simulations were carried out using a complex fiber mode solver from Photon Design (FIMMWAVE) to find the effective indices of the fiber cladding modes that tunnel across into the SPR. The simulated structure was made up of the SMF fiber, a gold coating of $20 \mathrm{~nm}$, and a thin dielectric coating with various thicknesses and refractive indices. In all cases pure water was used as the outer medium. The parameters used in the simulation are given in Table 1 (we used material refractive index values for a wavelength of $1545.335 \mathrm{~nm}$; the dispersion of the material was taken into account).

Table 1. Refractive indices of the materials used

\begin{tabular}{lllll}
\hline & Core & Cladding & Gold & Water \\
\hline Refractive index & 1.4504 & 1.4440 & $0.5194-$ & 1.3150 \\
& & & $\mathrm{i} 10.6821$ & \\
\hline Source & & {$[26]$} & {$[27]$} & {$[28]$} \\
\hline
\end{tabular}

For each value of coating thickness and refractive index, the mode solver calculates the real and imaginary parts of the effective index of all the cladding modes using a cylindrical Finite difference algorithm. The SPR-modified modes are identified by a larger value of the imaginary part of their effective index [5]. Fig. 8 shows two sets of cladding mode effective indices corresponding to the fiber without and with a dielectric coating, both immersed in water. A well-defined SPR peak consisting of a group of lossy cladding modes appears very clearly in each case. As can be seen from Fig.8, the position of the SPR peak shifts towards longer wavelengths if a coating with a refractive index higher than the refractive index of the solution in which the sensor is immersed was deposited. 
The simulations allowed us to plot the expected SPR effective index shift values with increasing coating thickness for various values of the refractive index of the coating (Fig. 9). In order to overlay the experimental results on the same graph and hence to determine which film refractive index provides the best fit to our data, we had to convert SPR wavelength shifts into effective index shifts and also assign a thickness value to each layer. Since SPR shifts were measured in water, the "dry" average final thickness measured with the AFM had to be converted into a fully hydrated thickness prior to adding the measured SPR vs thickness values on the simulation template. According to the experimental results, the total SPR wavelength shift due to the deposition of the $15 \mathrm{bi}$ layer structure was $5.99 \mathrm{~nm}$. Using Equation 3, this total shift corresponds to a change in plasmon effective index of 0.01 . For the thickness, the AFM result on the final dried film gave an average thickness of $16.8 \mathrm{~nm}$ but it is estimated that the coating was twice as thick when fully hydrated [29], i.e. approximately $33.6 \mathrm{~nm}$ of overall thickness or 2.24 nm per fully hydrated bi-layer.

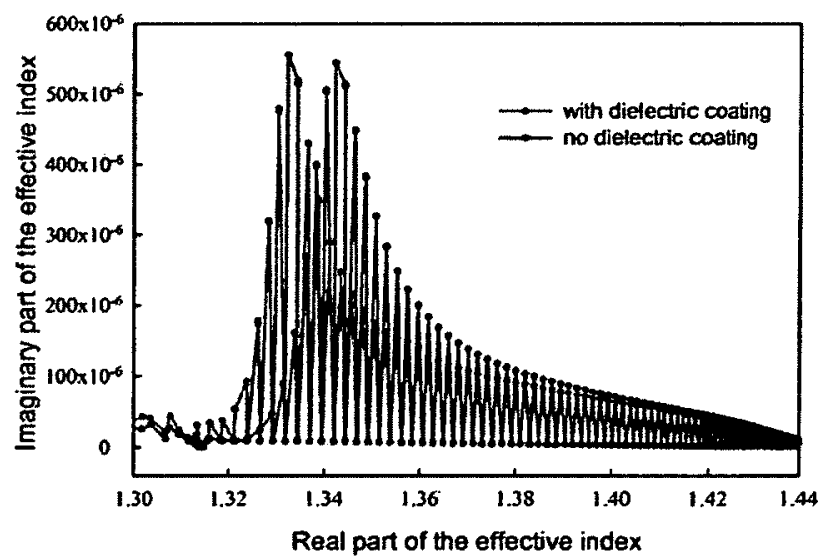

Fig. 8. Distribution of the effective indices of the modes (real and imaginary parts) supported by a gold-coated circular waveguide when (a) the waveguide was immersed in 
water and had no coating (pink curve); (b) and when the waveguide was immersed in water and had a $30 \mathrm{~nm}$ dielectric coating with a refractive index of 1.435, (black curve).

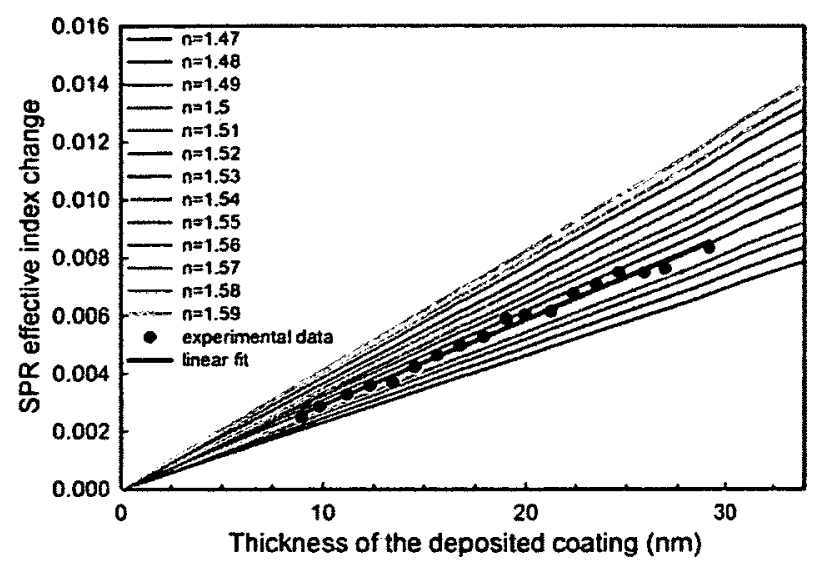

Fig. 9. Black dots: change of the plasmon effective index (from the position of the centre of the envelope used to fit SPR-coupled resonances) due to the deposition of thin dielectric film on the surface of the gold coating. Straight colored lines: simulated changes of the plasmon effective index as a function of the thickness of a deposited film, for various values of the film refractive index.

With these considerations taken into account, we get that for the regular portion of the film growth (roughly from 50 to 220 minutes on Fig. 5) the results of Figure 9 show that the average slope of the effective index change calculated from the experimental data matches best the effective index shifts expected from the deposition of a polymer with refractive index of 1.515 (hydrated). As a final note on these results, the response of the TFBG-SPR sensor that we use actually represents an average of several shorter measurements since the grating is $1 \mathrm{~cm}$ long and the plasmon propagation distance for our configuration is of the order of $500 \square \mathrm{m}$ (calculated from the peak values of the imaginary part of the effective index). Therefore, while the AFM scan revealed the highly irregular structure of the deposited film (Fig. 6(b)), our sensor measurement 
averages these irregularities and we feel confident that the SPR response can be interpreted using the average thickness of the coating determined by the AFM.

\section{Conclusion}

The results presented in this paper indicate that TFBG SPR sensors implemented in metal-coated standard telecommunication optical fiber are well suited for in situ characterization of the self-assembly of polyelectrolyte layers on the surface of an optical fiber. In the example provided, individual bi-layers had thicknesses of the order of 1.12 $\mathrm{nm}$ (once dried) and the sensor response provided real-time monitoring of the film growth during the deposition of 15 bi-layers with good linearity. A final, post-process AFM measurement on the deposited film provided the calibration factor between the thickness change and wavelength shift of the SPR resonance and also, indirectly through modeling of the SPR behavior, the refractive index of the final film (1.515 while fully hydrated in the process container). The overall results indicate that this fiber SPR sensor is also well suited to be used as a probe for the monitoring of liquid-phase deposition processes for various dielectric coatings on other substrates (of any form) in real time and in situ.

\section{Acknowledgments}

The authors would like to acknowledge support from the Natural Sciences and Engineering Research Council of Canada, the Canada Foundation for Innovation, LxDATA and the Canada Research Chair Program. 


\title{
Chapter 4
}

\subsection{Label-Free Biosensing with a Surface Plasmon Resonance Fiber Grating Aptasensor}

\author{
(published in Analytical Chemistry 2011) \\ Yanina Shevchenkol, Tariq J. Francis ${ }^{2}$, David A. D. Blair ${ }^{2}$, Ryan Walsh', Maria C. \\ DeRosa $^{2}$ and J. Albert ${ }^{*}$ \\ ${ }^{1}$ Department of Electronics, Carleton University, 1125 Colonel By Drive, Ottawa, K1S \\ 5B6, Canada \\ ${ }^{2}$ Department of Chemistry, Carleton University, 1125 Colonel By Drive, Ottawa, K1S \\ 5B6, Canada
}

\begin{abstract}
:
Surface Plasmon Resonance (SPR) biosensors prepared using optical fibers can be used as a cost-effective and relatively simple-to-implement alternative to well established biosensor platforms for monitoring biomolecular interactions in situ or possibly in vivo. The fiber biosensor presented in this study utilizes an unmodified single mode fiber as a transducing element with an in-fiber tilted Bragg grating used as the coupling element to excite the SPR on the surface of the sensor. The label-free biorecognition scheme used herein relied on the functionalization of the gold-coated sensor with aptamers, synthetic
\end{abstract}


DNA sequences that bind with a high specificity to a given target. In addition to being able to monitor in real time the functionalization of the fiber by the aptamers, the results show that the fiber biosensor is capable of detecting the presence of the aptamer's target, in this case thrombin, at various concentrations, both in buffer and serum solutions. It is also demonstrated that the SPR biosensor can be successfully applied for the evaluation of the dissociation constant $\left(K_{d}\right)$, since the binding constant agreed with values already reported in the literature.

\section{Introduction:}

The rapid and sensitive detection of analytes at low concentrations is paramount in many fields such as medicine, environmental monitoring and food safety. The use of compact and cost-effective solutions such as fiber sensors is appealing since they can be used for in situ and remote measurements using low cost equipment and sensing components. However, the performance of fiber sensors can be compromised by several issues associated with the design of the transducing mechanism and with difficulties arising from the need to modify the fiber geometry. These issues result in an insufficient sensitivity, a low reproducibility of the measurement performance and the fragility of the fabricated sensors. Thus, fiber-based biosensors have not yet been able to replace or compete for real-life applications with other biosensing platforms such as those relying on planar optical, piezo-electric or electronic transducing elements.

In this project, we are studying an innovative fiber biosensor platform that is able to overcome the challenges mentioned previously. It combines a number of technologies to create a simple and robust platform that has been successfully applied to a full range of 
target-analyte interactions. Compared to other technologies relying on bulk optical elements, the possibility of monitoring biomolecular interactions using fiber biosensors provides a unique opportunity for in situ biochemical measurements.

The sensing mechanism used in this study relies on the Surface Plasmon Resonance effect. SPR is a unique electro-magnetic phenomenon occurring at the interface between materials with opposite dielectric constants ${ }^{1}$. It allows for the detection of material changes occurring at the nanometer level in layers adjacent to this interface. As a result, SPR has been successfully applied for the characterization of various biomolecular reactions and their dynamics ${ }^{2}$. SPR platforms traditionally employed in biosensing use the total internal reflection of light on the base of a high refractive index prism to excite Plasmon waves, as in the so-called 'Kretschmann' configuration ${ }^{3}$. Although the prismbased SPR platform has a high sensitivity, it is rather costly, bulky and is limited because it can only be applied in a laboratory environment. Thus, SPR sensing platforms utilizing an optical fiber as a transducing element have gained considerable attention since it allows for the sensor design to be very compact and cost-effective. Another advantage is related to the use of a single-mode fiber, making it possible to operate remotely over large distances because there is no issue with the modal distribution of the guided light that reaches the sensor element. Compared to the commonly used prism-based SPR configuration, the implementation of SPR on the surface of an optical fiber is more challenging because light propagating in a fiber has to be first 'phase-matched' to the surface Plasmon waves. Secondly, the detection of chemical changes occurring on the surface of the sensor requires monitoring of very small changes of the coupling angle between the light propagating in the sensor and Plasmon waves on its external surface. 
This is not always feasible taking into account the significant spectral width of SPR coupling achieved in certain designs.

Traditionally, SPR excitation in optical fibers has been accomplished using either microstructured fibers ${ }^{4}$, standard fibers that were modified ${ }^{5-7}$ using in-fiber gratings or for the most part a combination of these features ${ }^{8}$. The use of microstructured or modified fibers, such as photonics crystal fibers, tapered, thinned or side-polished fibers, increases the difficulty in fabrication and cost while also diminishing the mechanical strength of the sensing elements. In most cases, fibers used in such designs are multimode waveguides. Therefore, it is still quite difficult to achieve precise control over SPR changes using the spectral dependence of the evanescent field coupling in large core multimode fibers. From this perspective, grating-assisted coupling of light from the core mode of a single mode optical fiber to selected cladding modes provides very narrow resonances in the transmission spectrum that are advantageous for the precise tracking of SPR changes. In-fiber gratings used for SPR excitation in a single mode fiber is also a key for maintaining its structural integrity ${ }^{9}$. One of the disadvantages associated with using a straight in-fiber grating, such as a Long Period Grating (LPG) or Fiber Bragg Grating (FBG) is their limited operating range. Operating range (or range of measurable refractive indices) of a grating-based sensor ${ }^{10}$ is proportional to the number of excited modes that could be coupled to SPR. Straight in-fiber gratings can excite only one fiber mode in a given spectral window. Thus the variation of the refractive index of ambient that can be detected by such a sensor type is limited by the spectral width of the single excited mode. 
The sensor presented here avoids all of the above issues by using a tilted Fiber Bragg Grating (TFBG). Since the TFBG is imprinted in the fiber core at a certain angle relative to the longitudinal axis of the fiber, it allows for a considerably wider operating range by exciting tens of claddings modes (Figure 1A), at unique well-defined wavelengths over a relatively modest spectral window. Each of the excited cladding modes can be potentially coupled to a SPR wave on the surface of the fiber if the SPR phase matching condition is satisfied, as determined by the refractive index of the medium immediately adjacent to the fiber surface ${ }^{11}$. We use an unmodified and inexpensive standard telecommunication single mode fiber (CORNING SMF-28) in these experiments, making the sensor very robust and simple to manufacture. Besides being robust, the single mode fiber has no issues with the modal distribution of the guided light that reaches the sensor element; this is something that could be observed in multimode fibers and could affect SPR spectral width. Furthermore, the single mode fiber's transmission loss is $0.17 \mathrm{~dB} / \mathrm{km}^{12}$ (or equivalently, less than $4 \%$ of power loss per $\mathrm{km}$ ) while the dynamic range for measuring the transmission of in-fiber devices with widely available telecommunication instrumentation (since we are using ordinary telecommunication fiber in its normal wavelength range) is well over $40 \mathrm{~dB}$ (or $99.99 \%$ ). Such low transmission losses could allow for remote operation over large distances (up to multi-km distances).

An additional advantage associated with using TFBGs is the possibility to eliminate the effect of the temperature cross-sensitivity from the sensor readings. In other kinds of SPR sensors, the well-known cross-sensitivity of the SPR coupling angle on temperature requires stringent temperature control during experiments and the presence of a temperature sensor or second sensing element. Although our metal-coated TFBGs are 
also sensitive to temperature variations, it is possible to eliminate this effect during the processing of the experimental data (even in real time). This is due to the invariance of the overall transmission spectrum of the TFBG to the temperature and the presence of one spectral resonance that is only dependent on the temperature that can be used as a high accuracy thermometer (with $0.1^{\circ} \mathrm{C}$ precision) ${ }^{13}$.

Having a wide operating range, a relatively high wavelength shift sensitivity of $455 \mathrm{~nm}$ per unit of refractive index (riu) ${ }^{11}$ and a very simple fabrication method has allowed this sensor platform to be successfully applied for chemical sensing and real-time in situ monitoring of polyelectrolyte multilayer formation ${ }^{14}$. The material presented here deals with applying the same sensor technology towards biosensing and it is one of the first complete and rigorous experimental studies of a SPR fiber biosensor utilizing a TFBG.

As molecular recognition is the cornerstone of biosensing, an essential component to any biosensor is the specific molecular recognition probe that can target the analyte of interest. While antibodies have been the gold standard for molecular recognition probes for several decades, the relatively new technology of aptamers is emerging as a more robust and cost-effective alternative. Aptamers are short oligonucleotide sequences that can be synthesized to bind with a strong affinity and specificity to a given molecular $\operatorname{target}^{15,16}$. Aptamers have already been successfully applied as molecular recognition probes for a variety of sensor techniques such as electrochemical ${ }^{17,18}$, piezoelectric $^{19,20}$ and optical sensors ${ }^{16,21,22}$. Prism-based SPR sensors have also been previously reported to use aptamers ${ }^{20,23,24}$. In the field of biosensing, one of the most promising advantages associated with the use of aptamers is the possibility of synthesizing aptamers that bind to a wide range of targets including drugs ${ }^{25}$, proteins ${ }^{26}$, or even supramolecular complexes 
such as viruses or bacteria ${ }^{27,28}$. Recently, an aptamer-modified biosensor ${ }^{29}$ using a preexisting and partially-automated SPR sensor platform was implemented in an etched multimode fiber ${ }^{5}$. Taking into account growing interest towards aptamers as highly modifiable and versatile biorecognition elements and the advantages of fiber-based SPR sensors, we were interested in studying the effectiveness of an aptamer-based SPR sensor implemented with our TFBG, standard single-mode fiber platform (See Figure 1). In this way, we sought to avoid the drawbacks of using a modified, multimode fiber, while preserving the ease of use, sensitivity, and selectivity of an aptamer-fiber system. Our experimental results indicate that our sensor can be used for the real-time monitoring of both the self-assembly of aptamers on the surface of a gold-coated fiber as well as for detection of protein molecules at various concentrations in solutions including serum. The dissociation constant $\left(K_{d}\right)$ of the target bound to the aptamer-sensor surface was determined and was found to be comparable to that found in solution studies. 


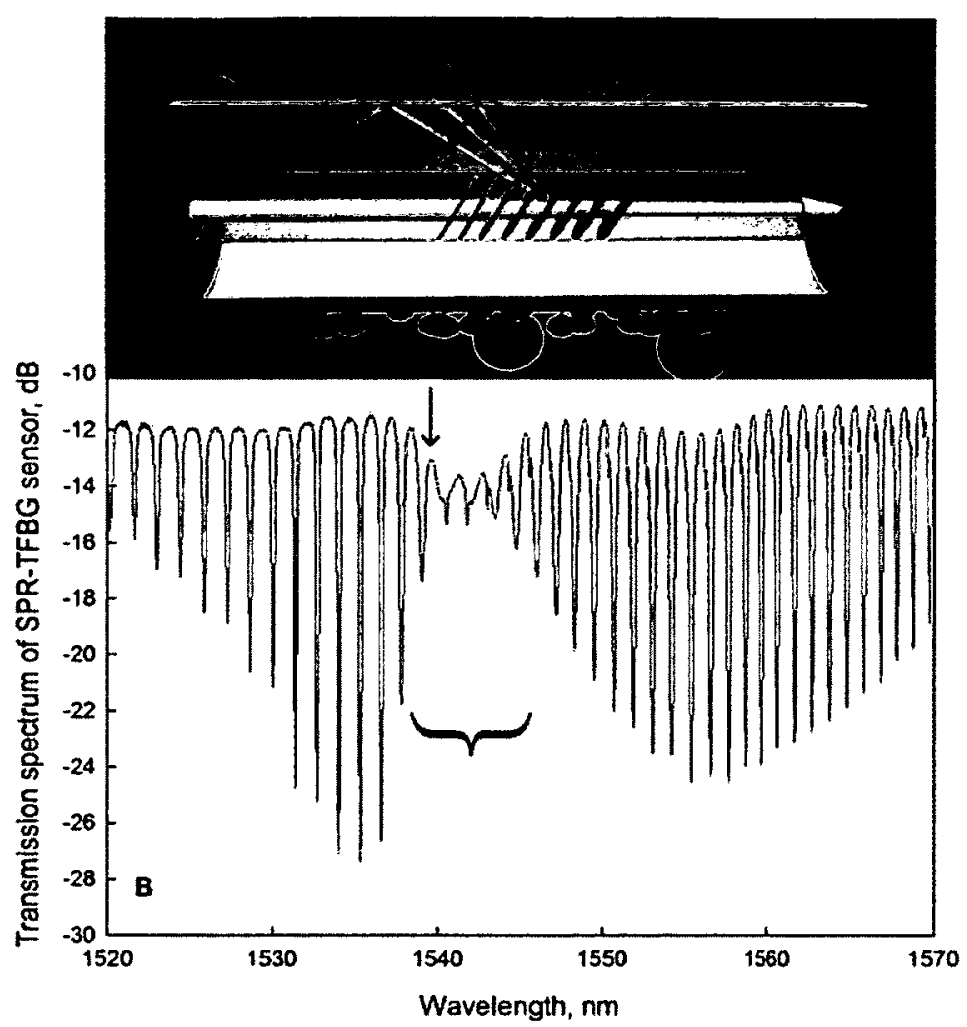

Figure 1. (A) 3D illustration of the presented SPR-TFBG biosensor: a standard optical fiber with a TFBG imprinted in its core coupling light to the SPR. A monolayer of thrombin aptamer probes (shown in red) immobilized on the gold coating interacts with their cognate thrombin protein targets (shown in blue). (B) Sensor's response measured in the aptamer solution, SPR-coupled part is shown with red brackets. The red arrow indicates the most sensitive SPR-coupled cladding resonance.

\section{Experimental section}

\section{Fabrication of the TFBG-SPR sensor:}

As reported earlier ${ }^{11,14,30}$, the SPR-TFBG sensor was manufactured using a standard single-mode fiber (Corning SMF-28) with a TFBG inscribed in the fiber's core and gold coating deposited on the surface of the fiber's cladding. TFBG was manufactured using 
the phase-mask technique: it required shining of UV light at $248 \mathrm{~nm}$ onto the surface of a bare fiber that was previously saturated with hydrogen. The grating's planes were written with a certain tilt relatively to the longitudinal axis of the fiber (Figure 1A). Tilt of the grating is an important parameter that can be used to choose which set of cladding modes is going to be excited. As a result, it makes it possible to adjust the operating range of the sensor tailoring it towards certain refractive indices. The gratings had a tilt of $10^{\circ}$, which allowed them to operate in aqueous solutions with refractive indices around 1.31-1.34.

A thin gold coating was deposited on the surface of the fiber after the gratings were inscribed. Deposition was conducted in a vacuum sputtering chamber and required two consecutive runs with the fiber being rotated by $180^{\circ}$ between depositions to ensure a more or less uniform coating around the circumference of the fiber. Thickness of the gold layer was optimized to have strongest SPR effect and was selected to be $50 \mathrm{~nm}$ for these experiments.

\section{Experimental setup, optical configuration:}

The experimental setup required the sensor to operate in the transmission regime. During the experiments, the sensors had to be fixed in a $7.5 \mathrm{~cm}$ by $2 \mathrm{~cm}$ plastic cell designed specifically for the biosensing tests. While each individual sensor was fixed in the platform with help of UV-sensitive adhesive, the sensing element $(0.5 \mathrm{~cm}$ in length) was immersed in a carved out indentation ( $45 \mathrm{~mm}$ by $5 \mathrm{~mm}$ ), which was then covered by a glass slide to avoid evaporation of fluids during the experiments. Immersion of the sensor was conducted at the beginning of each test using a pipette; on average $600 \mu \mathrm{L}$ of fluid was added. 
Sensors were interrogated using an Optical Sensing Analyzer (model Si720 from Micron Optics). Light over $1520-1570 \mathrm{~nm}$ range was linearly polarized using Polarization Controller from JDS Uniphase. Before each experiment the orientation of the polarization was adjusted to maximize coupling of the cladding modes to the SPR to ensure the strongest contrast between SPR-coupled and non-SPR modes. The measurement resolution was set at $0.0025 \mathrm{~nm}$ and measurements were recorded each 0.8 seconds. A schematic of the used optical setup can be found in the Supporting Information (Figure S1).

\section{Analysis of the experimental data:}

Figure 1B provides an example of the typical transmission spectrum obtained with the SPR-TFBG sensor while being immersed in the aptamer solution making it possible to excite the SPR. The red bracket in Figure 1B denotes the portion of the SPR-modified spectrum. The analysis presented in this paper is based on tracking the changes in the SPR-modified portion of the spectrum; more particularly tracking of the most sensitive SPR coupled cladding resonance. This resonance occurs on the left from the narrowest part of the SPR-coupled region, as indicated by the red arrow in Figure 1B. The power level of the maximum of that resonance was taken into account during evaluation of the SPR signal change.

Evaluation of the SPR signal change based on this method, where the absolute value of the position of only one resonance is considered, was applied for analysis of the data where the refractive index change was anticipated to be relatively small. Such experiments included simple one-step tests with a certain chemical reaction taking place 
on the surface of the sensor, such as self-assembly of aptamers or binding of target molecules to the aptamers. However, experiments where a larger refractive index change occurred required a different approach that would ensure consistency between the datasets obtained during different parts of the experiment. Such experiments, where the sensor was immersed in several solutions with different refractive indices, would be impossible to analyze taking into account the same SPR-coupled resonance because different solutions would result in different resonances being identified as the most sensitive. Thus, the analysis of such tests was based on tracking of the normalized amplitude value of that resonance. Such normalization ensured that the SPR changes happening in different solutions and affecting different resonances with different amplitudes could be compatible and comparable. Data analysis based on normalized value of the resonance's amplitude was applied for the $K_{d}$ evaluation (see below). All experimental data was smoothed by using a 400 -term LOESS filter ${ }^{31}$.

\section{Aptamer sequences, buffers, serum, thrombin protein and water:}

All buffer solutions were prepared using doubly deionized water from a Milli-Q water system (ThermoFisher). All DNA phosphoramidites and modifiers were purchased from Glen Research. Bovine Serum and Bovine Serum Albumin were purchased from Sigma Aldrich. Bovine serum solution for testing was prepared as a solution of $50 \%$ Serum: $50 \%$ Tris Buffer ( $50 \mathrm{mM}$ Tris, $1 \mathrm{mM} \mathrm{MgCl}, 140 \mathrm{mM} \mathrm{NaCl}, 5 \mathrm{mM} \mathrm{KCl}$ ). The thiolated thrombin aptamer, DNA sequence HS- $\left(\mathrm{CH}_{2}\right)_{6}-5$ '-GGT TGG TGT GGT TGG - 3' (herein called thrombin aptamer), was synthesized on a MerMADE 6 DNA synthesizer (Bioautomation Corporation). The sequence purification was conducted using Clarity 
QSP Cartridges (Phenomenex). The DNA was dissolved in "DNA buffer" (5 mM phosphate, $100 \mathrm{mM} \mathrm{MgCl}_{2}, 50 \mathrm{mM} \mathrm{NaCl}$ ). DNA sequences were confirmed by ESI-MS and the Ellman's test ${ }^{32}$ was conducted to test for presence of the 5' Thiol group prior to binding.

Human alpha-thrombin protein was purchased from Haematologic Technologies Inc. The protein was aliquoted as needed and dissolved in "Protein Buffer" (50 mM Tris, $1 \mathrm{mM} \mathrm{MgCl}, 140 \mathrm{mM} \mathrm{NaCl}, 5 \mathrm{mM} \mathrm{KCl}$ ).

The sequences used for the confocal microscopy experiments were purchased from Alpha DNA (Montreal). The thrombin aptamer sequences possessed the 5'-thiol'C6 modifier, as well as a Cyanine $3\left(\mathrm{Cy} 3^{\mathrm{TM}}\right)$ dye modifier at the $3^{\prime}$ end, making the overall sequence 5'-HS-( $\left(\mathrm{CH}_{2}\right)_{6}$-GGT TGG TGT GGT TGG-Cy3-3' (herein called Cy3 DNA).

\section{Immobilization of the thrombin aptamer:}

Prior to the immobilization of the thrombin aptamer on the surface of the sensor, the sensors were washed with ethanol and then with Milli-Q water. After the cleaning step, DNA functionalization was conducted by filling out the carved indentation with the aptamer solution of the desired concentration. Transmission spectra were collected automatically every 0.8 seconds during the entire experiment. Aptamer deposition times and concentrations varied between tests. After the aptamer adsorption step was complete, the sensor was rinsed with DNA buffer several times before the protein solution was added. 


\section{Binding of the thrombin protein to the thrombin aptamer:}

Thrombin solutions were prepared to a desired concentration using human $\alpha$-thrombin and Protein Buffer. The solutions were used to fill the cut out indentation of the platform. The duration of soaking in the thrombin solution varied between 20 min and 2 hours depending on the experiment. After immersion in the protein solution, the sensor was washed with Protein Buffer for several minutes to prepare for the next step.

\section{Confocal microscope imaging:}

Confocal microscope imaging combined with fluorescent labelling was used to evaluate and confirm the self-assembly of the aptamer on the surface of the gold coating of the sensors.

All confocal microscopy images were collected on a Zeiss LSM510 with a PlanAcochromat 63x/1.4 Oil Dic objective with LP950 filter, with an excitation wavelength of $550 \mathrm{~nm}$ and an emission wavelength of $570 \mathrm{~nm}$.

Before running the experiment, the sensors were prepared by rinsing them with anhydrous ethanol and then with DNA Buffer. Cy3 DNA was dissolved in DNA Buffer to make a $45 \mu \mathrm{M}$ solution. Sensors were then immersed in the aptamer solution for 20 hours, rinsed thoroughly, and later imaged while mounted on microscope slides in a solution of $50 \%$ glycerol in water, and covered with $1.7 \mu \mathrm{m}$ thick cover slides.

\section{Atomic Force Microscope (AFM) Imaging:}

AFM imaging was conducted in order to evaluate the surface of the sensor after binding to thrombin protein. All AFM images were collected on an Ntegra system using a 
SFC050LNTF AFM Head on an inverted microscope (Olympus IX71) (See Supporting Information for details regarding AFM tools). Before imaging, gold-coated fibers were immersed in a $25 \mu \mathrm{M}$ solution of thiolated thrombin aptamer for a period of 4 hours. The fiber was then rinsed with DNA buffer and immersed in a $30 \mu \mathrm{M}$ solution of $\alpha$-thrombin in Protein Buffer for approximately 12 hours. After immersion, the fibers were rinsed with Milli-Q water and dried in air before imaging. All samples were collected in air on the dry samples.

\section{Results and discussion:}

\section{Monitoring of thrombin-aptamer self-assembly:}

Results of the experiments that involved soaking of the sensor in the aptamer solutions of varying concentrations are shown in Figure 2. Raw SPR data is shown in the sensorgram in Figure 2A along with the filtered signal in order to illustrate a difference between both types of signals. As it can be seen, the signals obtained in the solutions with aptamers showed a considerable increase over the first 6 to 8 minutes of soaking (Figure $2 \mathrm{~A}$ ), and then showed a much slower change, suggesting that initial binding of the aptamer to the surface occurs quickly. Moreover, the signal obtained from soaking in the DNA buffer was significantly lower than the signal obtained in the aptamer solutions. Doubling the aptamer concentration from 10 to $20 \mu \mathrm{M}$ did not lead to a doubling of the signal, suggesting that $20 \mu \mathrm{M}$ aptamer is concentrated enough to saturate the surface for our experiments. 


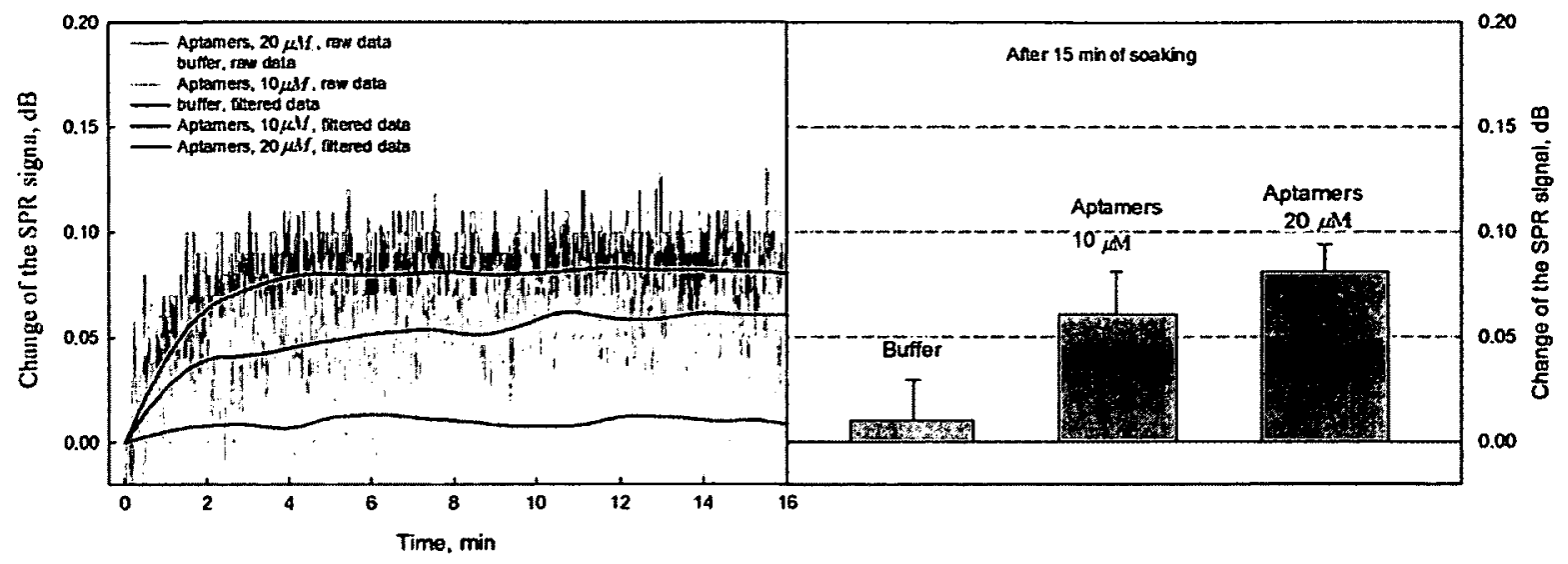

a)

b)

Figure 2. (A) Real-time SPR signal changes over the first 15 minutes of aptamer selfassembly versus soaking in the DNA buffer and Milli-Q water, raw SPR signal is shown along with filtered data. (B) Bar diagram illustrating difference in SPR signals after first 15 min of soaking in solutions.

Attachment of the aptamers was verified using confocal fluorescent imaging (Figure 3A). DNA modified with the fluorescent $\mathrm{Cy} 3$ dye was immobilized on the surface of the sensor and was imaged after unbound aptamers were rinsed away during the washing step. A fluorescent signal coming from the fiber surface can clearly be seen in the case of the Cy3 DNA modified surfaces. As a control, a bare sensor immersed in a solution of free $\mathrm{Cy} 3$ dye showed no fluorescence (see supporting documentation, Figure S3 A-B). These results confirm the immobilization of the modified thrombin aptamer sequence on the surface of the fiber. 


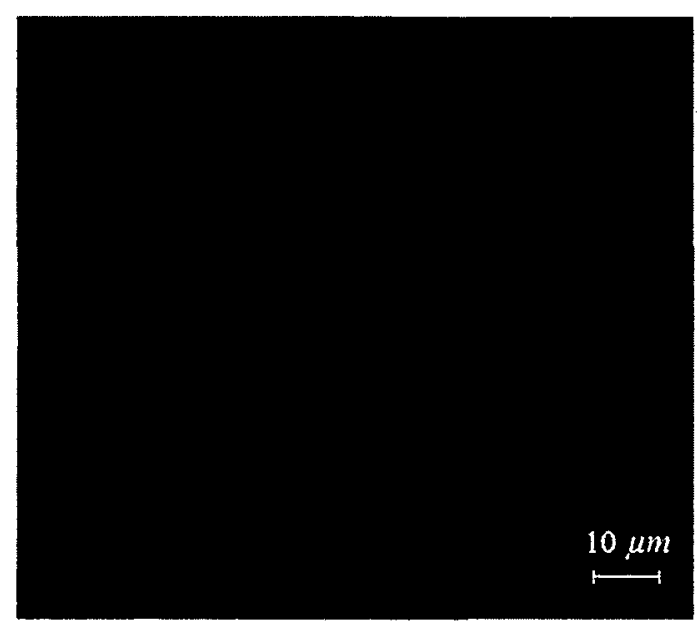

A

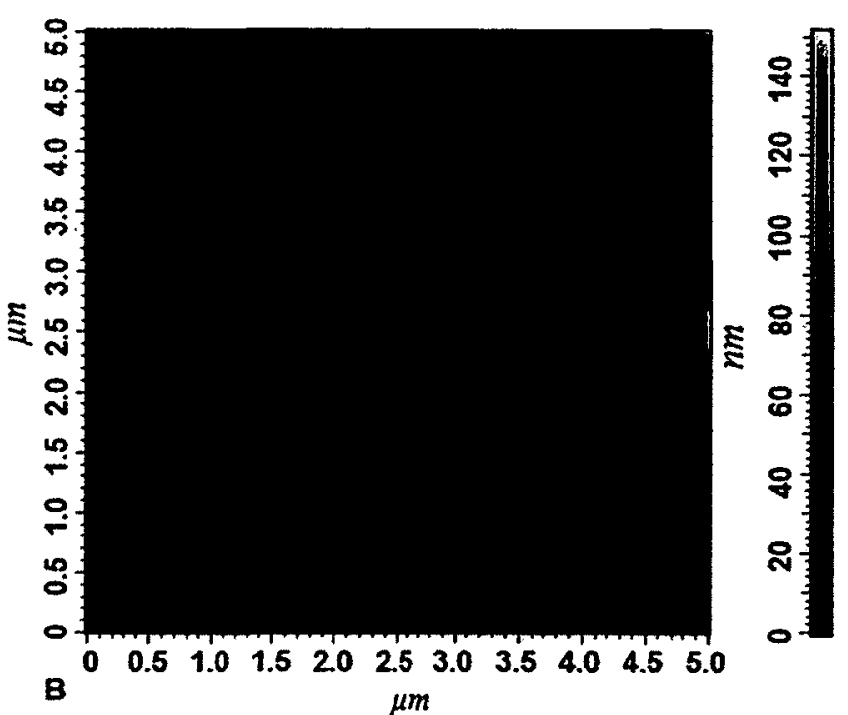

Figure 3. (A) Confocal microscope image of an optical fiber immersed in a $45 \mu \mathrm{M}$ of Cy3- and thiol-modified DNA solution for 20 hours. $11 \%$ laser strength, detector pinhole width $55 \mu \mathrm{m}, 63 \mathrm{X}$ magnification were applied. (B) AFM image of sensor's surface after attachment of the thrombin (tapping mode, measured in air on the dry sample).

\section{Detection of the thrombin target:}

Results of the immersion of the aptamer-functionalized sensor into the thrombin solutions are shown in the Figures $3 \mathrm{~B}$ and 4 . Figure $4 \mathrm{~A}$ indicates that, similarly to soaking in DNA solution, the SPR signal changes rapidly within the first $10 \mathrm{~min}$ of immersion and then stabilizes. In addition to the signal comparison with Protein Buffer, several control solutions were also examined. BSA is typically used as a control for thrombin aptamer studies ${ }^{33}$ however, it is much larger than thrombin which can lead to some non-specific binding (67 kDa compared to thrombin's $37 \mathrm{kDa}$ ). Pepsin, which is comparable in size to thrombin $(35 \mathrm{kDa})$ and should have no affinity for the thrombin 
aptamer, was also examined in order to gain a better perspective regarding non-specific binding. Comparison of the thrombin data against that generated by soaking in the BSA, pepsin, and buffer solutions indicates that selective detection of target molecules at concentrations that are as low as $0.1 \mu \mathrm{M}$ is possible. A difference in signals can be detected in as little as 10 minutes (Figure 4A). However, after 100 minutes the signals have stabilized and show the greatest discrimination between specific and non-specific signals (Figure 4B).

Evaluation of the sensor's response in a more complex solution such as serum is also shown in the bar diagram, Figure 4B. The results for soaking in serum and serum mixed with protein show that, despite a strong non-specific signal increase in the presence of serum, the attachment of thrombin can still be detected. The absolute SPR signal difference between the $50 \%$ serum solution and the $5 \mu \mathrm{M}$ thrombin-serum solution (approximately $0.07 \mathrm{~dB}$ ) is very similar to the difference between buffer and $5 \mu \mathrm{M}$ thrombin-buffer solution $(0.074 \mathrm{~dB})$ Thus, the specific thrombin-aptamer interactions can be effectively detected, even in complex media.

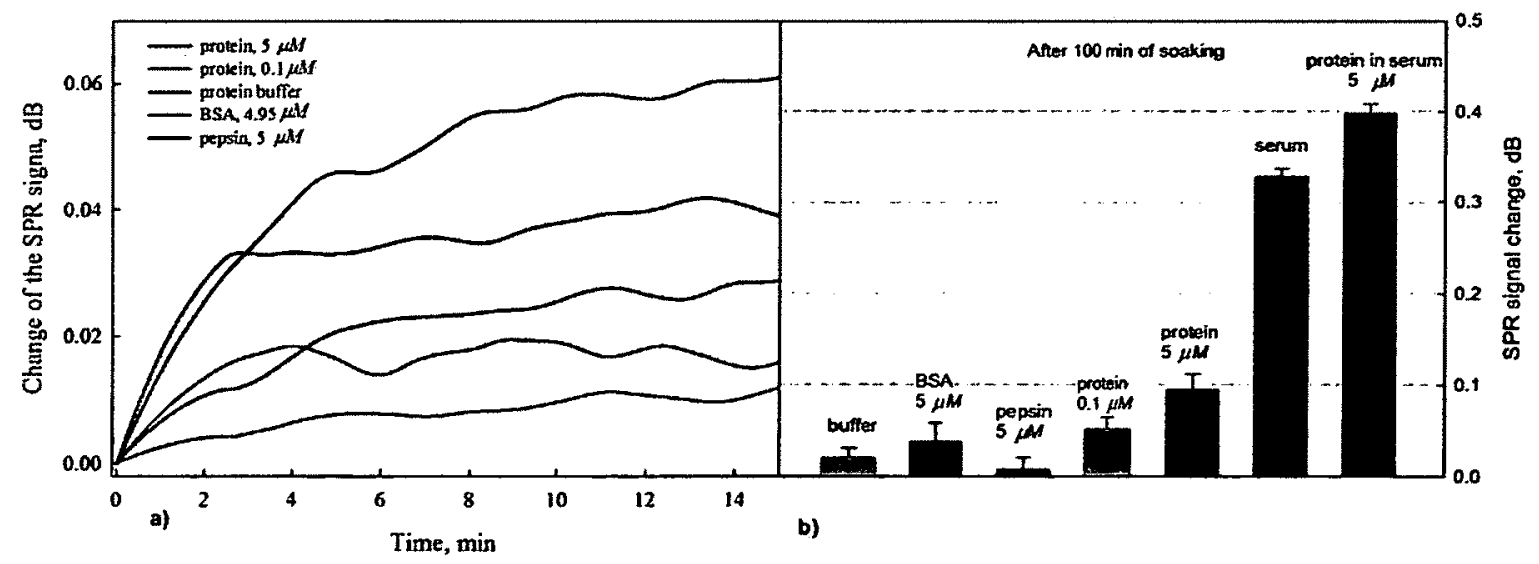


Figure 4. (A) Real-time SPR response measured during the first 15 minutes of soaking in 0.1 and $5 \mu \mathrm{M}$ thrombin solutions versus soaking in $5 \mu \mathrm{M}$ BSA, $5 \mu \mathrm{M}$ pepsin and Protein Buffer. (B) Bar diagram comparing SPR response abtained in serum and protein sultions after soaking for $100 \mathrm{~min}$.

The attachment of protein was confirmed by imaging the sensor's surface using AFM (Figure 3B). AFM reveals that the scanned sensor's surface has many new structural features with sizes at the $\mu \mathrm{m}$ and sub- $\mu \mathrm{m}$ scale that are not present on the aptamer modified surface alone (see Supporting Information for 3D AFM images, Figures 4S AC). It is anticipated that a molecule of thrombin protein has a dimension of $4.5 \times 4.5 \times 5$ $\mathrm{nm}^{34}$, which is much smaller than the observed features. A possible explanation of observed features could be that the protein molecules formed clusters in the solution before or during the interaction with aptamer molecules. In the latter case, formation of protein clusters on the surface of the sensor could be a result of the uneven distribution of aptamer molecules on the surface of the sensor, a finding that has been previously reported $^{35}$.

Even though the thrombin molecules did not form a uniform layer on the surface of the sensor, measured SPR signal changes should be proportional to the overall protein amount adsorbed to the surface of the grating. The SPR is excited over the full length of the grating, which is $1 \mathrm{~cm}$, therefore, the observed SPR response was averaged over the length of the TFBG that was used to excite the SPR.

The signal to noise ratio (SNR) varied from 0.5 to 3.97 for experiments with aptamers (presented in the Figure 2B) and from 0.36 to 19.83 for experiments with thrombin (presented in the Figure 4B), depending on the experiment. Errors were found as a 
standard deviation between the data points of same SPR data set when the signal was saturated and leveled off. There was a relatively high noise level because of the intrinsic noise of the interrogation system. The SNR could be significantly improved by selecting a more stable light source with a lower level of noise.

\section{$K_{d}$ analysis and sensor's characterization:}

The dissociation constant $\left(K_{d}\right)$ is a widely used parameter applied to characterize binding strength between two interacting molecules. The motivation behind using a fiber biosensor for determining $K_{d}$ constants stems from the interest in developing a portable sensor platform that can be applied to the in situ measurement of biomolecular kinetic interactions without perturbing the surrounding environment.

The dissociation constant $\left(K_{d}\right)$ for the thrombin aptamer has been reported earlier, based on studies by ${ }^{36-38}$ be between 20 and $200 \mathrm{nM}$. However, it is well known that the functionalization of aptamers to surfaces may alter the binding characteristics of the system $^{39}$. Therefore, the characteristics of the immobilized aptamers and their effect on the binding dynamics had to be assessed.

Evaluation of the $K_{d}$ constant was performed using an analyte "Ladder" test. Initially the sensor was functionalized with the aptamer followed by its immersion in successive solutions of increasing concentrations of the target. Figure 5 shows the results of one ladder experiment where the sensor was immersed in a set of solutions with thrombin concentration increasing from $0.1 \mu \mathrm{M}$ to $5 \mu \mathrm{M}$. The SPR signal (raw and smoothed data) was obtained by normalizing the amplitude of the most sensitive SPR-coupled resonance. Figure 5 illustrates the correlation between increase in SPR signal and deposition of the 
aptamer (step 3). This was followed by subsequent soaking of the sensor in solutions of increasing thrombin concentration (steps 4-7). Washing with protein buffer (step 8) did not lead to a dramatic loss of signal, confirming the specific interaction between the protein and the sensor. Removal of the biomolecular layer was achieved by soaking in sodium carbonate (step 9). The inset in Figure 5 illustrates the relationship between normalized SPR signal change and thrombin concentration that was used to determine $K_{d}$. It was found that the $K_{d}$ for the sensor was approximately $40 \pm 1 n M$ indicating that attachment of the aptamer to the gold surface of the sensor did not significantly affect the binding affinity between the aptamer and thrombin. See Supporting Information, Figure S5, for $K_{d}$ SPR data and for details regarding the model fit applied to evaluate the $K_{d}$ constant.

This suggests that this method of sensor generation offers the potential for cheap portable alternatives to the bulky expensive systems usually associated with analytical detection methods. 


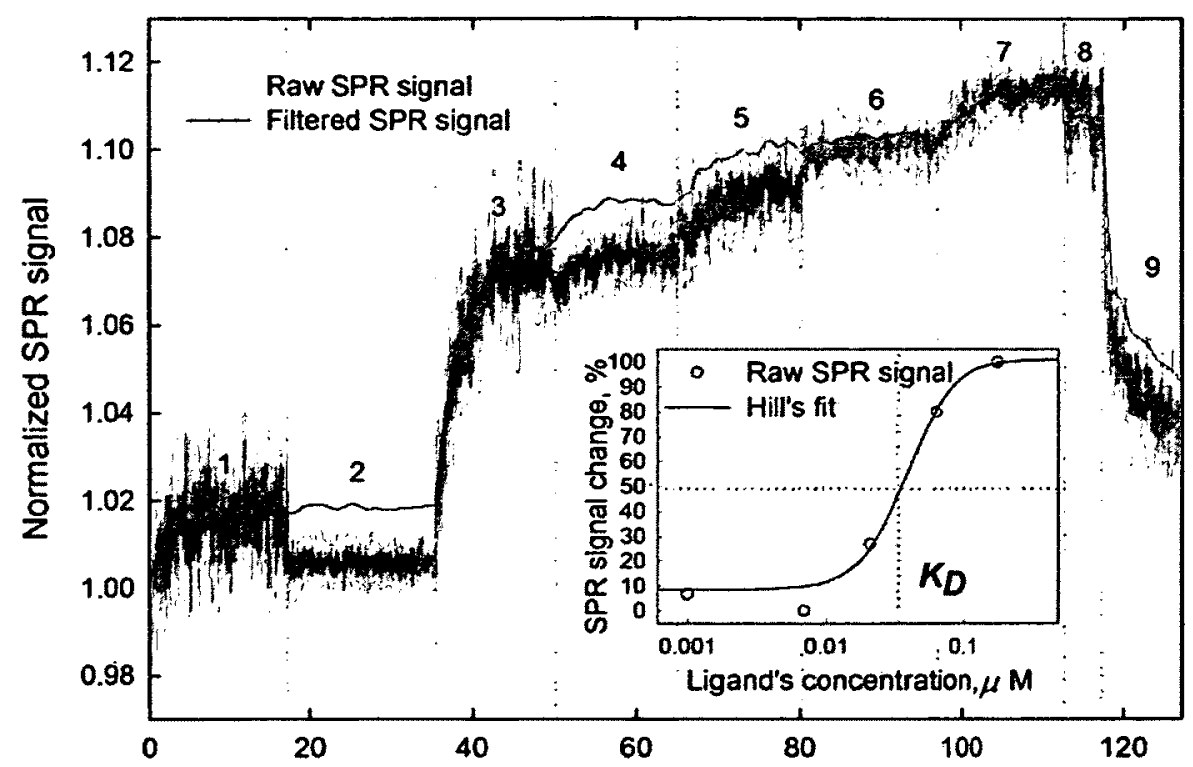

Figure 5. SPR signal change during the $K_{d}$ - type test ('Ladder' test). Description of each step: 1. MilliQ water; 2. DNA buffer; 3. Aptamers, concentration $20 \mu \mathrm{M} ; 4$. Thrombin, concentration $0.1 \mu \mathrm{M} ; 5$. Thrombin, concentration $0.5 \mu \mathrm{M} ; 6$. Thrombin, concentration 1 $\mu \mathrm{M}$; 7. Thrombin, concentration $5 \mu \mathrm{M}$; 8. Protein buffer; 9. Regeneration in $0.2 \mathrm{M}$ of Na2CO3. Inset: Evaluation of the $K_{d}$ constant using relation between ligand's concentration and SPR signal. The data set was normalized to the maximum of the SPR signal and was fitted using Hill's equation ${ }^{40}$ (see Supporting Documentation for information regarding the fitted model and $K_{d}$ experiment's data).

\section{Conclusions}

This study demonstrates for the first time how a TFBG-SPR sensor manufactured from an unmodified single mode fiber can be used as a biosensor to detect a biomolecular target at varying concentrations. The results showed that the TFBG-SPR sensor could be successfully applied to biosensing studies making this biosensor platform a strong alternative to the existing biosensing modalities that rely on optical and non-optical 
transducing elements. More specifically, this system can be used for the real-time quality control of sensor manufacturing as the self-assembly of the sensor can be monitored. It was reported that aptamers, which were used as the biorecognition element, attach themselves to the surface providing the same signal observed in the detection of the analyte itself. This system provided a robust detection of thrombin in buffer and more complex solutions such as serum. The signal difference between specific and non-specific interactions remained the same regardless of the solution, which suggests several possibilities for future work in complex environments.

The findings confirmed that the biosensor can be applied for traditional real-time characterization of biorecognition element-target interactions, as well as, for a multi-step analysis to determine the $K_{d}$ constant. The value of $K_{d}$ was found to be well within the range of values already reported in the literature. These findings together suggest that this sort of sensor platform could be used for the in situ characterization of biochemical reactions which makes it a viable alternative to other more costly or bulky wellestablished analytical tools.

\section{ASSOCIATED CONTENT}

Supporting Information. Additional information as noted in text.

\section{ACKNOWLEDGEMENTS}

This work was supported by the Natural Sciences and Engineering Research Council of Canada, Canada Research Chair Program, the Ontario Early Researcher Award and by

LxData Inc., Montreal. We would like to thank Dr. Anatoli Ianoul and Graham Galway for 
assisting with the AFM imaging of the sensor samples. We would also like to acknowledge Anne-Fook Yang, Denise Chabot and Yasir Sultan from Agriculture Canada for helping us acquire the confocal fluorescent images.

\section{REFERENCES}

(1) Raether, H. Surface Plasmons on Smooth and Rough Surfaces and on Gratings; Springer-Verlag: Berlin, Germany, 1988.

(2) Homola, J. Chem. Rev. 2008, 108, 462-493.

(3) Kretschmann, E.; Raether, H. Z. Naturforsch 1968, A 23, 2135-2136.

(4) Hassani, A.; Skorobogatiy, M. J. Opt. Soc. Am. B. 2009, 26, 1550-1557.

(5) Jorgenson, R.C.; Yee, S.S. Sensor. Actuat. B-Chem. 1993, 12, 213-220.

(6) Allsop, T.; Neal, R.; Mou C.; Mapps, D.; Bennion, I.; Kalli K.; Webb, D.J. Appl. Optics 2009, 48, 276-286.

(7) Tubb, A. J. C.; Payne, F. P.; Millington, R.; Lowe, R. C. Electron. Lett. 1995, 37, 1770-1770.

(8) Nemova, G.; Kashyap, R. J. Mater. Sci: Mater. Electron. 2007, 18, s327-s330.

(9) He, Y.; Lo, Y.; Huang, J.J. Opt. Soc. Am. B. 2005. 23, 801-811.

(10) Slavik, R.; Homola J. Sensor. Actuat. B-Chem. 1999, 54, 74-79.

(11) Shevchenko, Y.Y.; Albert, J. Opt. Lett. 2007, 32, 211-213.

(12) Corning®, 2010. Corning® SMF-28® ULL Optical Fiber with Corning® Ultra LowLoss Technology. $\quad$ < http://www.corning.com/WorkArea/showcontent.aspx?id=14357> (accessed on Aprl $15,2010)$.

(13)Shao, L.Y.; Shevchenko Y.; Albert, J. Opt. Express. 2010, 18, 11464-11471.

(14) Shevchenko, Y.; Ahamad, N. U.; Ianoul, A.; Albert J. Opt. Express. 2010, 18, 2040920421.

(15) Rimmele, M. Chembiochem. 2003, 4, 963-971. 
(16) Cho, E.J.; Lee, J.; Ellington, A. D. Annu. Rev. Anal. Chem. 2009, 2, 241-264.

(17) Ikebukuro K.; Kiyohara C.; Sode K. Biosens. Bioelectron. 2005, 20.2168-2172.

(18) So H. M.; Won K.; Kim Y.H.; Kim B-K.; Ryu B.H.; Na P.S.; Kim H.; Lee J.-O. J. Am. Chem. Soc. 2005, 127, 11906-11907.

(19) Liss M.; Petersen B.; Wolf H.; Prohaska E. Anal. Chem. 2002, 74, 4488-4495.

(20) Tombelli S.; Minunni M.; Luzi E.; Mascini M. Bioelectrochemistry 2005, 67, 135141.

(21) Jhaveri S.D.; Kirby R.; Conrad R.; Maglott E.J.; Bowser M.; Kennedy R.T.; Glick G.; Ellington A.D. J. Am. Chem. Soc. 2000, 122, 2469-2473.

(22) Stojanovic M. N.; De Prada P.; Landry D.W. J. Am. Chem. Soc. 2001, 123, 49284931.

(23) Ostatná, V.; Vaisocherová H.; Homola J.; Hianik T. Anal. Bioanal. Chem. 2008, 391, 1861-1869.

(24) Wang J.; Zhou H.S. Anal. Chem. 2008, 80, 7174-7178.

(25) Xiao, H.; Edwards, T.E.; Ferre-DèAmare, A.R. Chemistry and Biology 2008, 15, 1125-1137.

(26) Keefe, A.D.; Pai, S.; Ellington, A. Nat. Rev. Drug Discovery 2010, 9, 537-550.

(27) Bruno, J.G.; Carrillo, M. P.; Phillips, T.; Andrews, C.J. J. Fluoresc. 2010, 20, 12111223.

(28) Jeon, S.H.; Kayhan, B.; Ben-Yedidia, T.; Arnon, R. J. Biol. Chem. 2004, 279, 4841048419.

(29) Pollet J.; Delport F.; Janssen K. P. F.; Jans K.; Maes G.; Pfeiffer H.; Wevers M.; Lammertyn J. Biosens. Bioelectron. 2009, 25, 864-86.

(30) Shevchenko Y.; Chen C.; Dakka M. A.; Albert J. Opt. Lett. 2010, 35, 637-639.

(31) Cleveland, W.S. J. American Statistical Association 1979, 74, 829-835.

(32) Ellman, G.L. Arch. Biochem. Biophys. 1959, 82, 70-77.

(33) Rahman, M.A.; Son, J.I.; Won, M.; Shim, Y. Anal. Chem. 2009, 81, 6604-6611.

(34) Bode W.; Mayr, I.; Baumann, U.; Huber, R.; Stone, S.R.; Hofsteenge, J. The EMBO Journal 1989, 8, 3467-3475. 
(35) Ajore, R.; Kumar, R.; Kaur, I.; Sobti, R.C.; Bharadwaj, L.M. J. Biochem. Biophys. Methods. 2007, 70, 779-785.

(36)Bock L. C.; Griffin L. C.; Latham J. A.; Vermaas E. H.; Toole J. J. Nature 1992, 355, 564-566.

(37)Hamaguchi N.; Ellington A.; Stanton M. Anal. Biochem. 2001, 294, 126-131.

(38)Potyrailo R.A.; Conrad R.C.; Ellington A.D; Hieftje G.M. Anal. Chem. 1998, 70, 3419-3425.

(39) Jing M.; Bowser M.T. Anal. Chim. Acta 2011, 686, 9-18.

(40) Goutelle S.; Maurin M.; Rougier F.; Barbaut X.; Bourguignon L.; Ducher M.; Maire P.; Fundamental\&Clinical Pharmacology 2008, 22, 633-648. 


\title{
4.2 Supplementary Information for 'Label-Free Biosensing
}

\section{with a Surface Plasmon Resonance Fiber Grating Aptasensor'}

\author{
Yanina Shevchenkol, Tariq J. Francis ${ }^{2}$, David A. D. Blair ${ }^{2}$, Ryan Walsh ${ }^{2}$, Maria C. \\ DeRosa ${ }^{2}$ and J. Albert ${ }^{l}$ \\ ${ }^{1}$ Department of Electronics, Carleton University, 1125 Colonel By Drive, Ottawa, K1S \\ 5B6, Canada \\ ${ }^{2}$ Department of Chemistry, Carleton University, 1125 Colonel By Drive, Ottawa, K1S \\ 5B6, Canada
}

\begin{abstract}
:
Surface Plasmon Resonance (SPR) biosensors prepared using optical fibers can be used as a cost-effective and relatively simple-to-implement alternative to well established biosensor platforms for monitoring biomolecular interactions in situ or possibly in vivo. The fiber biosensor presented in this study utilizes an unmodified single mode fiber as a transducing element with an in-fiber tilted Bragg grating used as the coupling element to excite the SPR on the surface of the sensor. The label-free biorecognition scheme used herein relied on the functionalization of the gold-coated sensor with aptamers, synthetic DNA sequences that bind with a high specificity to a given target. In addition to being able to monitor in real time the functionalization of the fiber by the aptamers, the results show that the fiber biosensor is capable of detecting the presence of the aptamer's target,


in this case thrombin, at various concentrations, both in buffer and serum solutions. It is also demonstrated that the SPR biosensor can be successfully applied for the evaluation of the dissociation constant $\left(K_{d}\right)$, since the binding constant agreed with values already reported in the literature.

We submit the following information as supplemental information:

1. Information about used optical setup;

2. Additional information about AFM configuration;

3. Confocal Control image;

4. 3D AFM images of the sensor's surface;

5. $K_{d}$ graph;

6. Information about fitting model applied to evaluate $K_{d}$ constant;

\section{The optical setup}

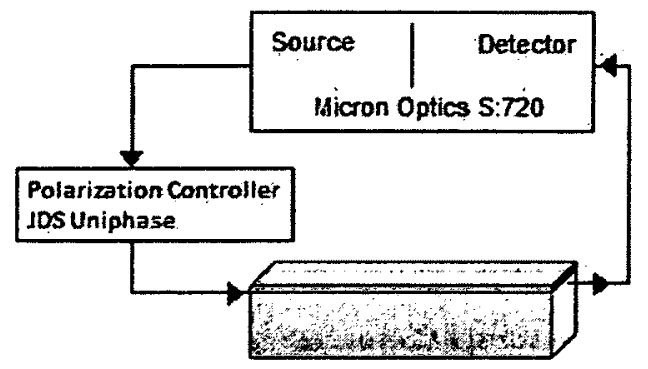

FIG. S1: Schematic of used optical setup including Micron Optics interrogation unit (si720), optical polarizer and sensor element immersed in the plastic platform. 


\section{Additional information about AFM configuration:}

All AFM images were collected in a dynamic mode using Tap300AI scanning probes purchased from BudgetSensors. The probes had a length of $125 \mu \mathrm{m}$, a mean width of 30 $\mu \mathrm{m}$, and thickness of $4 \mu \mathrm{m}$. Each probe's tip's height was $17 \mu \mathrm{m}$, and its radius was less than $10 \mathrm{~nm}$.

\section{Confocal control image of the bare sensor's surface:}

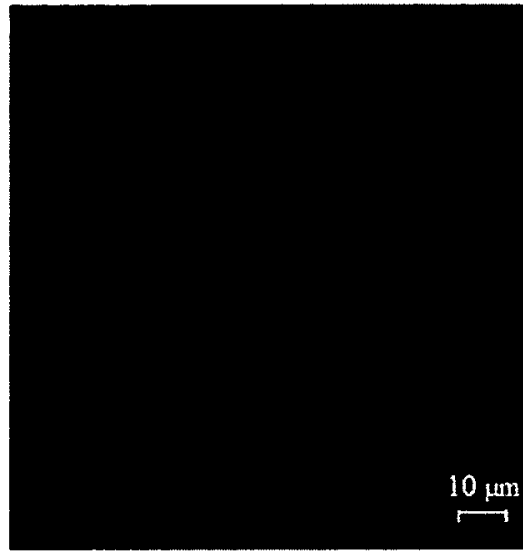

n)

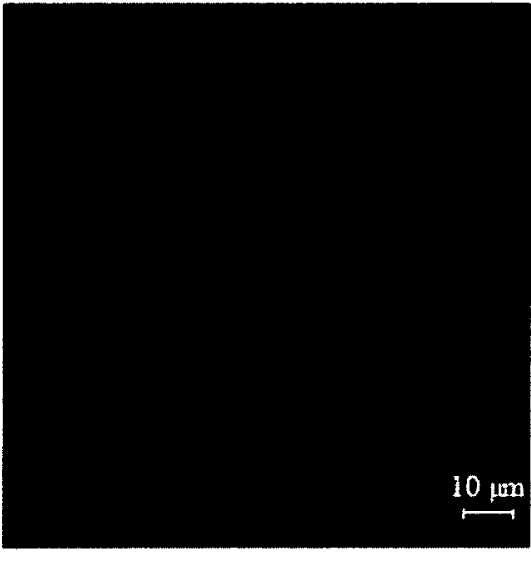

b)

FIG.3S Confocal microscope images of optical fiber immersed in a $30 \mu \mathrm{M}$ solution of Cy3-dye for 20 hours: a) $11 \%$ laser strength, detector pinhole width $55 \mu \mathrm{m}, 63 \mathrm{X}$ magnification; b) $80 \%$ laser strength, detector pinhole width $55 \mu \mathrm{m}$, $63 \mathrm{X}$ magnification. 
4. 3D AFM images of the sensor's surface:

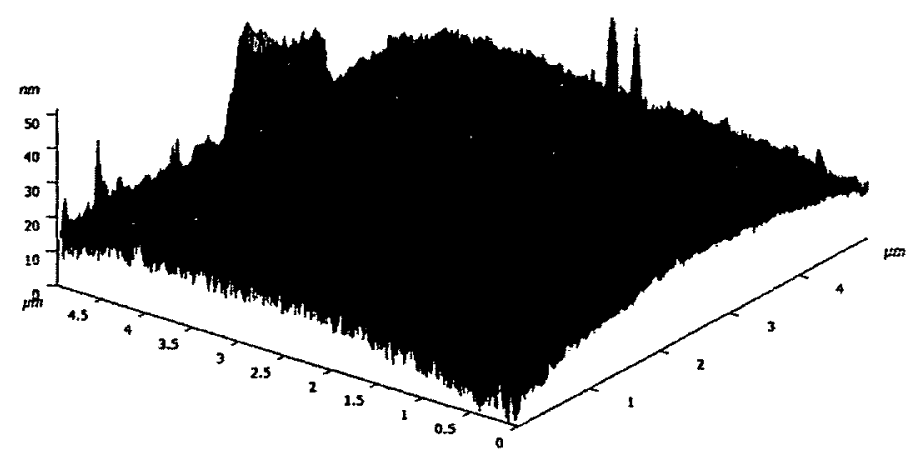

(A)

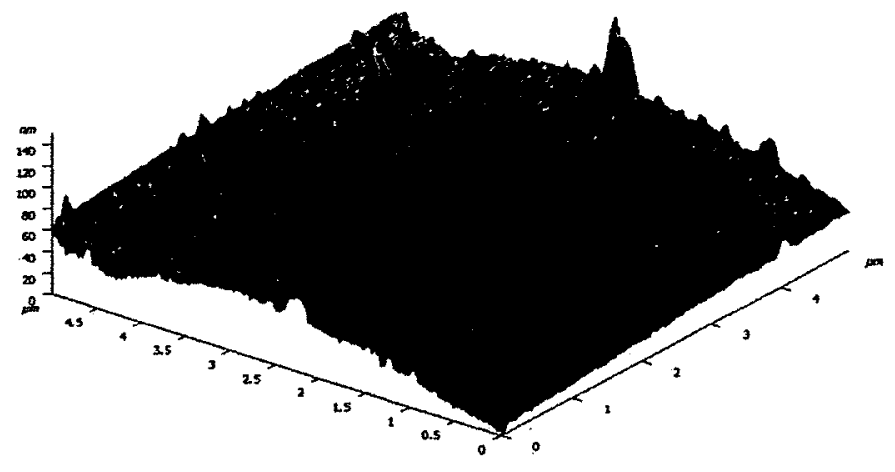

(B)

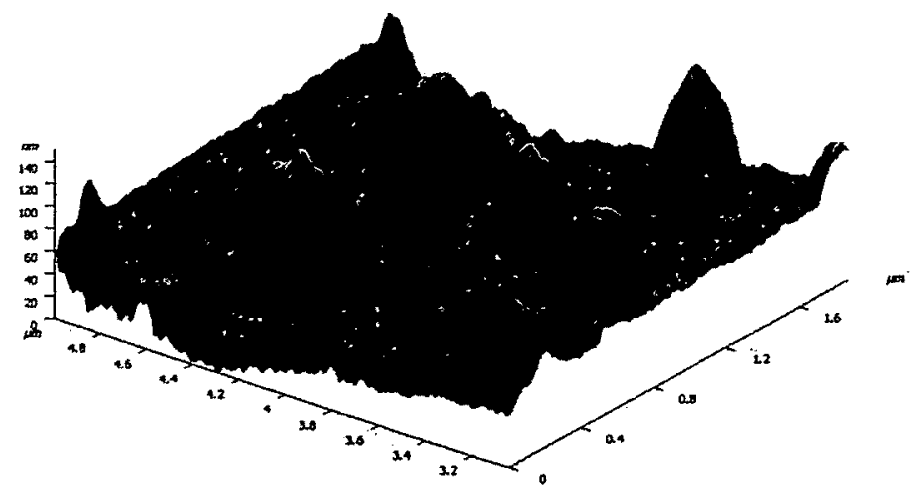

(C)

FIG.4S (A) 3D AFM image of the sensor's surface after soaking in the thiolated thrombin aptamer solution (B, C) 3D AFM images of the sensor's surface after attachment of thrombin (shown at different scales). 


\section{5. $K_{d}$ plot:}

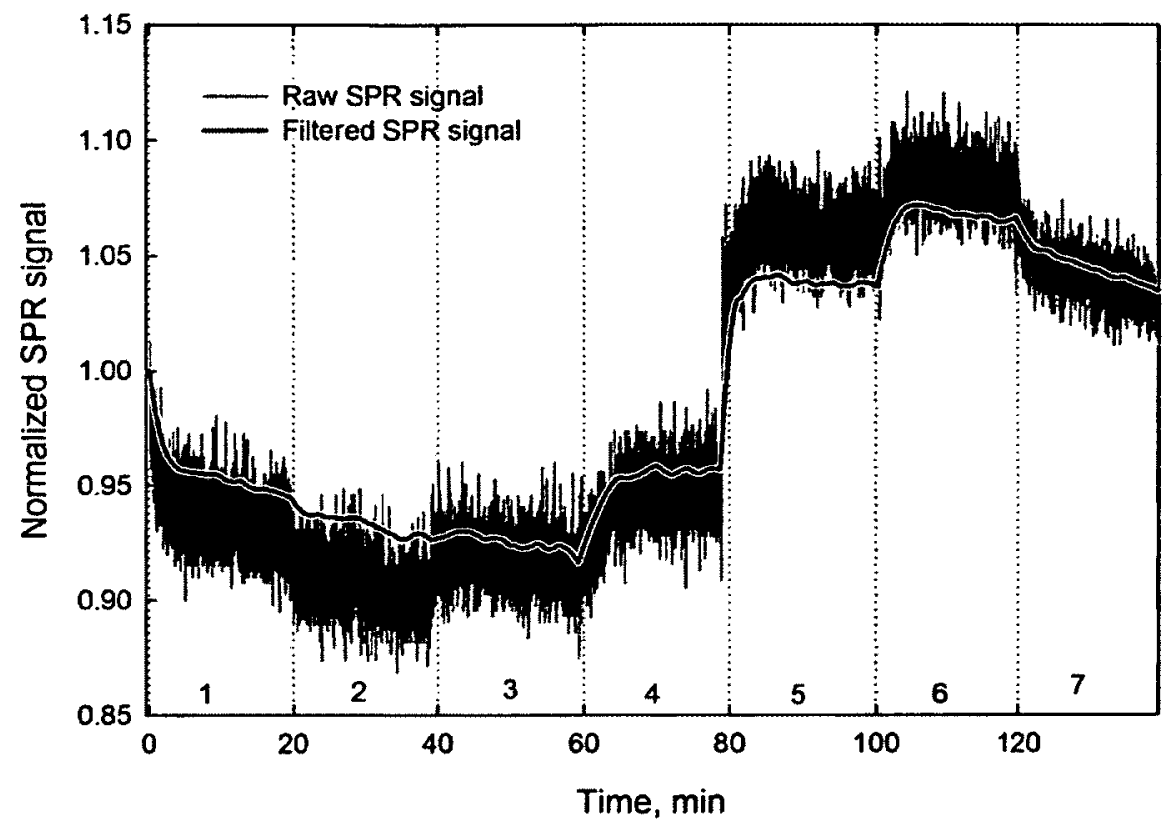

FIG 5S. $K_{d}$ signal, raw data is shown along with smoothed data. Description of each step:

1. Protein Buffer; 2. Thrombin, concentration $0.00685 \mu \mathrm{M} ; 3$. Thrombin, concentration $0.0205 \mu \mathrm{M} ; 4$. Thrombin, concentration $0.0616 \mu \mathrm{M} ; 5$. Thrombin, concentration 0.185 $\mu \mathrm{M} ; 6$.Thrombin, concentration $0.55 \mu \mathrm{M} ; 7$. Thrombin, concentration $1.66 \mu \mathrm{M}$.

\section{Information about fitting model used to calculate $\boldsymbol{K}_{\boldsymbol{d}}$ constant:}

Hill's equation has extensively been employed in pharmacology to study drug-receptor interaction $^{40}$. It has been applied to fit our experimental data presented in the Figure $5 \mathrm{~S}$ and Figure 5 (Inset) to evaluate $K_{d}$ constant:

$$
y=y_{0}+\frac{a x^{b}}{e^{b}+x^{b}}
$$




\section{Chapter 5}

\section{Surface Plasmon Resonance Fiber Sensor for Real-Time and}

\section{Label-Free Monitoring of Cellular Behavior}

(Submitted to Analytical Chemstry 2012)

Yanina Shevchenko, ${ }^{a, b, c}$ Gulden Camci-Unal ${ }^{b, c}$, Davide F. Cuttica ${ }^{b, c}$, Mehmet R. Dokmeci b,c, Jacques Albert ${ }^{a}$ and Ali Khademhosseini ${ }^{* b, c, d}$

${ }^{a}$ Department of Electronics, Carleton University, Ottawa, Canada. Fax: +1-613-5205708;

Tel: +1-613-5202600 x5578; E-mail: jacques_albert@carleton.ca

${ }^{b}$ Center for Biomedical Engineering, Department of Medicine, Brigham and Women's

Hospital, Harvard Medical School, Cambridge, MA 02139, USA. Fax: +1-617-7688477;

Tel: +1-617-7688395; E-mail: alik@mit.edu

${ }^{c}$ Harvard-MIT Division of Health Sciences and Technology, Massachusetts Institute of Technology, 77 Massachusetts Avenue, Cambridge, MA 02139, USA.

${ }^{d}$ Wyss Institute for Biologically Inspired Engineering, Harvard University, Boston, MA 02115, USA.

Keywords: Cellular analysis, plasmonic fiber sensor, cellular response, real time and label free sensing 


\section{Abstract:}

This paper reports on the application of an optical fiber biosensor for real-time analysis of cellular behavior. Our findings illustrate that a fiber sensor manufactured from traditional telecommunication fiber can be integrated into conventional cell culture equipment and used for the real-time and label-free monitoring of cellular responses to chemical stimuli. The sensing mechanism used for the measurement of cellular responses is based on the excitation of Surface Plasmon Resonances on the surface of the optical fiber. In this proof of concept study, the plasmonic fiber sensor was utilized to study the effect of a number of different stimuli on cells. In particular, we tested trypsin to induce cell detachment from the sensor's surface, serum to monitor its uptake by cells, and sodim azide to inhibit cellular metabolism. The effect of these different stimuli on cellular morphology was confirmed with several imaging tools. The SPR data was validated by alamar blue assay phase contrast and fluorescence microscopy. The results indicated that the fiber biosensor can be successfully utilized for real-time and label-free monitoring of cellular changes in the first 30 minutes following addition of a stimulus. Furthermore, we demonstrated that these optical fiber biosensors can be easily regenerated for repeated use, proving this platform as a versatile and cost-effective sensing tool.

Optical fiber sensors have been widely applied for the detection of a broad range of biological targets such as, nucleic acids ${ }^{68}$, antigens $s^{69}$, antibodies ${ }^{70}$ and other low and high molecular weight analytes. Recently there has been an increasing emphasis on the 
characterization of larger and more complex biological targets including cells, bacteria ${ }^{71}$ and viruses ${ }^{72}$. The use of cells as an integral part of the biorecognition scheme is driven by a growing demand for a better understanding of newly discovered cellular signaling pathways, a need for the characterization of drug-cell interactions as well as for a comprehensive evaluation of cellular interactions with their microenvironments ${ }^{73,74}$.

Optical label-free characterization of cellular behavior can be accomplished via several approaches. One approach is to use traditional imaging tools where light propagates in free space and interacts with cells positioned on its path. For instance, imaging using traditional cell phone cameras has been demonstrated as a cost-effective solution that could be integrated into cell culture incubators to monitor cellular responses induced by drugs and other stimuli ${ }^{65,75}$.

Another strategy to characterize cellular behavior is to use optical analytical tools where cells are in direct contact with optical elements used to confine and guide light. The detection of cellular reactions within the attached cells is made possible by the evanescent field of the light being confined by the optical elements: this evanescent field decays exponentially away from the boundary up to a distance of a few micrometers, sufficiently deep to penetrate into the cells and probe their chemical state. Monitoring the attenuation or phase of the light due to the interaction with cells can be used to interpret intra- and extra cellular changes while the cells are exposed to various physical or chemical stimuli. In particular, changes in the optical field can be used to monitor cellular adhesion, detachment ${ }^{64}$, death ${ }^{4}$, contraction, as well as triggering intracellular reactions ${ }^{8}$, ${ }^{64}$ that are very challenging to monitor with other means. So far, the use of such 
analytical tools for cellular characterization has been limited to a few optical platforms such as, the well-established Surface Plasmon Resonance (SPR) $)^{3-4,2,1}$ and resonant waveguide grating EPIC $®$ systems ${ }^{7-8,64}$. The signal captured from these sensors is labelfree, real-time, dose-dependent and can be easily quantified.

Although these optical systems can be used to characterize a wide range of cellular responses, the platforms themselves are large and are not designed to be integrated into tissue culture incubators. Handling of cells outside the incubators during their analysis limits cell viability and affects cellular metabolism. Moreover, operation of the platforms mentioned above is complicated and requires highly trained personnel. In this context, optical sensor platforms that are made using optical fiber offer several competitive advantages such as compactness, remote sensing capabilities, simple integration into cell culture equipment, and potential for in situ measurements. When the fiber sensors operate at standard telecommunication wavelengths, as in our case, both the fiber devices and interrogation equipment become highly cost-effective compared to more complex and bulkier custom designed optical sensing platforms. Taking into account that the sensitivities reported in the literature for fiber sensors can be similar to that of traditional platforms employing planar optical structures ${ }^{76}$, fiber sensors position themselves as a versatile solution that can be used in a range of applications, such as in situ whole cell sensing where bulky platforms would be disadvantageous.

Yanase et al. (2010) showed that a plasmonic fiber biosensor could be used for monitoring cellular response when cells are exposed to a stimulus, i.e. albumin ${ }^{29}$. The sensor employed in that study was manufactured using a multimode quartz fiber. 
However, the use of multimode fiber restricts sensor detection levels as well as hinders the quality of the detected optical signal. In this study we propose to use a completely different sensor manufactured using a standard telecommunication single mode fiber in order to improve upon these results. The use of traditional single mode fiber allows the fiber sensor to be very robust while achieving a high level of performance. Moreover, this is the first optical fiber sensor that was fully integrated into conventional cell culture equipment making it possible to conduct the experiments in a controlled environment. By conducting all measurements in a standard incubator, we obtained high cell viability and we were able to monitor cellular responses for prolonged periods of time.

Herein we present the application of a fiber biosensor, a miniature and portable sensor platform, for real-time and label-free monitoring of cellular responses. Particularly, we demonstrate that the plasmonic fiber sensor can be used for the characterization of a set of fine cellular responses triggered by selected stimuli. The sensor is manufactured using single mode fiber and works through the excitation of plasmon waves on its gold coated surface.

\section{EXPERIMENTAL SECTION}

\section{Materials}

Fibronectin was obtained from BioLegend (San Diego, CA, USA). Phosphate buffered saline (PBS), Dulbecco's modified Eagle medium (DMEM), fetal bovine serum (FBS), 10X trypsin and penicillin/streptomycin were purchased from Gibco (Carlsbad, CA, USA). Alamar Blue, Calcein AM, Ethidium homodimer, phalloidin (Alexa-Fluor 594), and 4',6-diamidino-2-phenylindole (DAPI) were obtained from Invitrogen Corp. 
(Carlsbad, CA, USA). Bovine serum albumin (BSA) and sodium azide was supplied by Sigma-Aldrich (St. Louis, MO, USA). Paraformaldehyde was obtained from Electron Microscopy Sciences (Hatfield, PA, USA). 4-well plastic plates were purchased from Fisher Scientific Co LLC (Pittsburg, PA, USA). All reagents were used as received without further purification.

\section{Fabrication of the Plasmonic Sensors}

The sensors used in this work (Figure 1A) were manufactured using a traditional telecommunication single mode fiber (CORNING SMF28) ${ }^{45,48}$. As reported earlier, the fabrication of sensor elements was completed in a two-step process that involved the fabrication of a resonant grating element followed by a deposition of a gold coating on the surface of the fiber. The gratings were imprinted in the hydrogen-loaded fiber by using UV light shined through a diffractive phase mask, which had a period of $1096 \mathrm{~nm}$. The UV beam was imposed on a fiber with an excimer laser operating at a wavelength of $193 \mathrm{~nm}$. After the inscription process, the gratings were annealed with a heat gun at $400^{\circ} \mathrm{C}$ for approximately $1 \mathrm{~min}$ and thermally stabilized in the oven at $120^{\circ} \mathrm{C}$ for 12 hours. The final step was the deposition of a $50 \mathrm{~nm}$ thick gold coating on the surface of the fiber. The deposition was implemented using an electron beam evaporation setup (Balzer Evaporator system).

\section{Principle of Operation of the Plasmonic Fiber Biosensor}

The fiber sensor operates through the excitation of the SPR effect on the surface of the gold-coated fiber ${ }^{45,48,30,51}$. A schematic of the sensor with cells attached to its surface is shown in Figure 1A. The SPR excitation is achieved by means of a tilted grating 
imprinted in the fiber core. The Tilted Fiber Bragg grating (TFBG) acts as a resonant element redirecting light from the guided core to the surface of the fiber. When the wavelength of the light propagating in the fiber is such that a phase matching condition for the excitation of the SPR is satisfied, some of the light escapes from the cladding and is coupled to plasmonic waves on the outer surface of the gold-coated fiber.

The surface plasmon wave is confined at the interface between gold coating and dielectric outside fiber (or sensed medium) with the evanescent tail of the plasmonic field penetrating deeply in the dielectric. It provides the whole excitation system with an enhanced sensitivity towards refractive index changes that occur near the surface. When cells are attached to the surface of the gold coating, the plasmonic field penetrates into the cells and enables the monitoring of chemical changes occurring inside them. The depth of penetration of the plasmonic field is proportional to the wavelength used to excite the $\mathrm{SPR}^{35}$ and is approximately $1400-1500 \mathrm{~nm}$ for this sensor. The measured SPR signal results from the average of all chemical changes occurring on the surface of the sensing element which covers $3.92 \mathrm{~mm}^{2}$ (for a $1 \mathrm{~cm}$-long grating) with a depth of penetration of $1500 \mathrm{~nm}$. 
A)
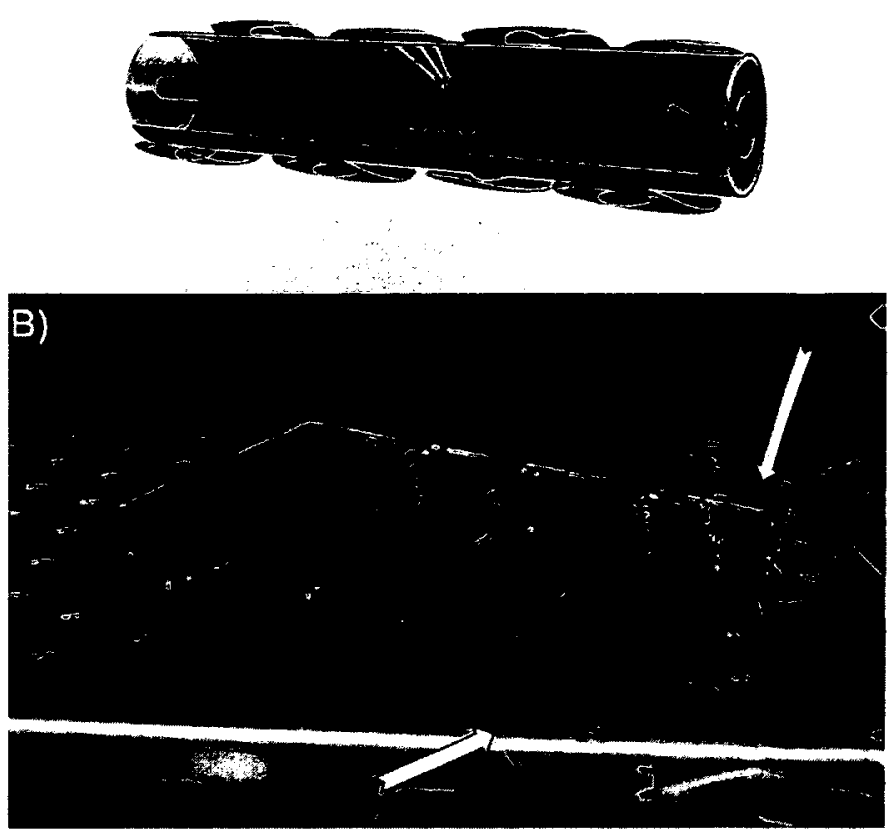

c)

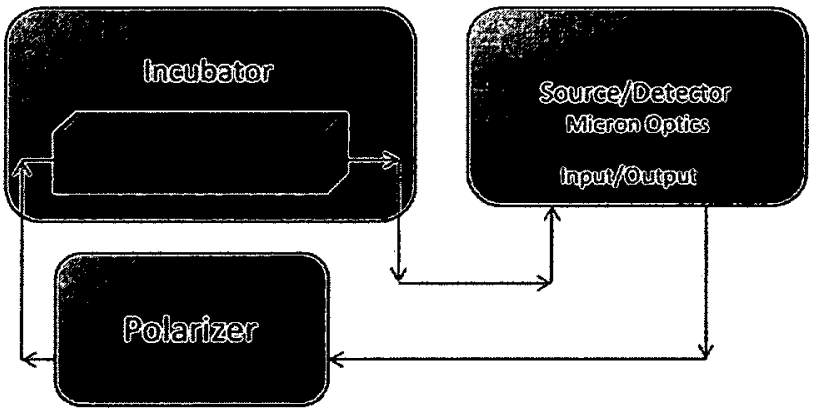

Figure 1. Optical fiber configuration. (A) 3D illustration of a fiber sensor with cells attached to the surface of the gold coating. Fringes in the core of the fiber indicate the tilted grating that redirects the light towards the surface of the fiber; (B) Photograph of a 4-well plate with an integrated fiber sensor during a typical experiment. Arrows point at optical fiber wired outside the plate; (C) Schematic of the optical interrogation setup (all the line arrows represent single mode fiber connections). 


\section{Optical Instrumentation and Sensor Integration into an Incubator}

All sensors were fixed on the bottom of rectangular 4-well plates using a UVcurable adhesive (Thorlabs, Newton, NJ, USA). The fiber tails of the sensors were guided through two holes made in the sidewalls of the plates (Figure 1B). Plates with sensing elements were placed in an incubator while the tails of the sensors were wired outside the incubator and connected to the interrogation setup (Figure 2C). The interrogation unit consisted of an Optical Sensing Analyzer (model Si720 from Micron Optics) and a Polarization Controller from JDS Uniphase. Measurements of the optical transmission spectrum of the devices were saved every $0.2 \mathrm{sec}$ with the spectral resolution set at $0.0025 \mathrm{~nm}$. The collected data was internally processed by the Micron Optics unit with a $10 \mathrm{kHz}$ filter.

\section{Cell Cultures}

The NIH-3T3 fibroblast cells were maintained in DMEM medium supplied by $10 \%$ FBS and $1 \% \mathrm{Pen} / \mathrm{Strep}$. Cell cultures were maintained in a $5 \% \mathrm{CO}_{2}$ regulated incubator at $37^{\circ} \mathrm{C}$. Media was changed every other day during cell culture period. Upon reaching 70$80 \%$ confluence, cells were either passaged into new flasks or harvested by trypsin and counted at the desired cell density ( 1 million cells $/ \mathrm{mL}$ ) to be used in experiments with the SPR-TFBG sensors.

\section{Sensor Modification and Monitoring Cellular Response}

To ensure cell attachment on the sensor surface, the gold coating was modified with fibronectin protein, which is a well known cell-adhesive reagent ${ }^{77}$. The fiber sensors were sterilized under UV light and washed with PBS six times before the surface modification. 
After aspiration of PBS, $500 \mu \mathrm{L}$ of $100 \mu \mathrm{g} / \mathrm{ml}$ fibronectin was placed on the sensors for one hour to facilitate the interaction of the gold coating and charged functionalities of the protein. Fibronectin was then removed, the sensors were rinsed with PBS and subsequently seeded with $5 \mathrm{~mL}$ of NIH-3T3s at a $2.1 * 10^{5}$ cells/ $/ \mathrm{cm}^{2}$ density. The sensors were incubated for two hours and the media was changed. Later, the sensors were incubated for an additional $10 \mathrm{~h}$ at $37^{\circ} \mathrm{C}$ to enable formation of a monolayer of cells on the surface of the fibers.

The experimental routine included recording of the sensor's output for $30 \mathrm{~min}$ before the addition of a stimulus, followed by aspiration of old media and addition of an agent in fresh media. The amounts of agent mixed with media were substantially smaller than the total amount of added media: therefore no detectable refractive index change was recorded by the sensor as a result of the addition of a new mixture. In such conditions, all SPR signal changes observed right after addition of stimuli were caused by the cellular responses rather than a bulk refractive index mismatch. Measurements of the SPR signal were conducted for 60 minutes after the aspiration and the addition of each of the stimuli.

After the experiments, sensor surfaces were regenerated by incubating with trypsin and ethanol. Sensors were first rinsed with PBS, then exposed to trypsin (10X) for 30 minutes and later left in ethanol solution (70\%) for 10 minutes. The regeneration step was completed by washing the sensors with PBS.

\section{Cellular Assays and Sample Imaging}

Live/Dead test, Alamar Blue assay and DAPI/Phalloidin staining were carried out following manufacturer's protocols. Alamar Blue assay was read using a Fluostar 
fluorescence plate reader supplied by BMG LABTECH GmbH (Offenburg, Germany) at $544 \mathrm{~nm} / 590 \mathrm{~nm}(\mathrm{Ex} / \mathrm{Em})$. Images were acquired using an inverted fluorescent microscope (Nikon Model TE 2000-U, Nikon instruments Inc., USA). Spot Advanced Software 4.6.4.6 supplied by Diagnostic Instruments, Inc. (Sterlington Heights, MI, USA) was used for capturing images.

\section{Statistical Analysis}

Statistical analysis of Alamar Blue data was performed using one-way analysis of variance (ANOVA) with Bonferroni post-test processing using the GraphPad software by Prism Inc. (La Jolla, CA, USA). For the analysis, the level of significance was set at $p<0.05\left({ }^{* * *} p<0.001,{ }^{* *} p<0.01,{ }^{*} p<0.05\right)$.

\section{RESULTS AND DISCUSSION}

\section{SPR Data Analysis in the Context of Cellular Analysis}

Figure 2 shows typical transmission spectra acquired with an SPR-TFBG sensor as well as an exemplary data analysis of the response of the sensor covered with cells. 

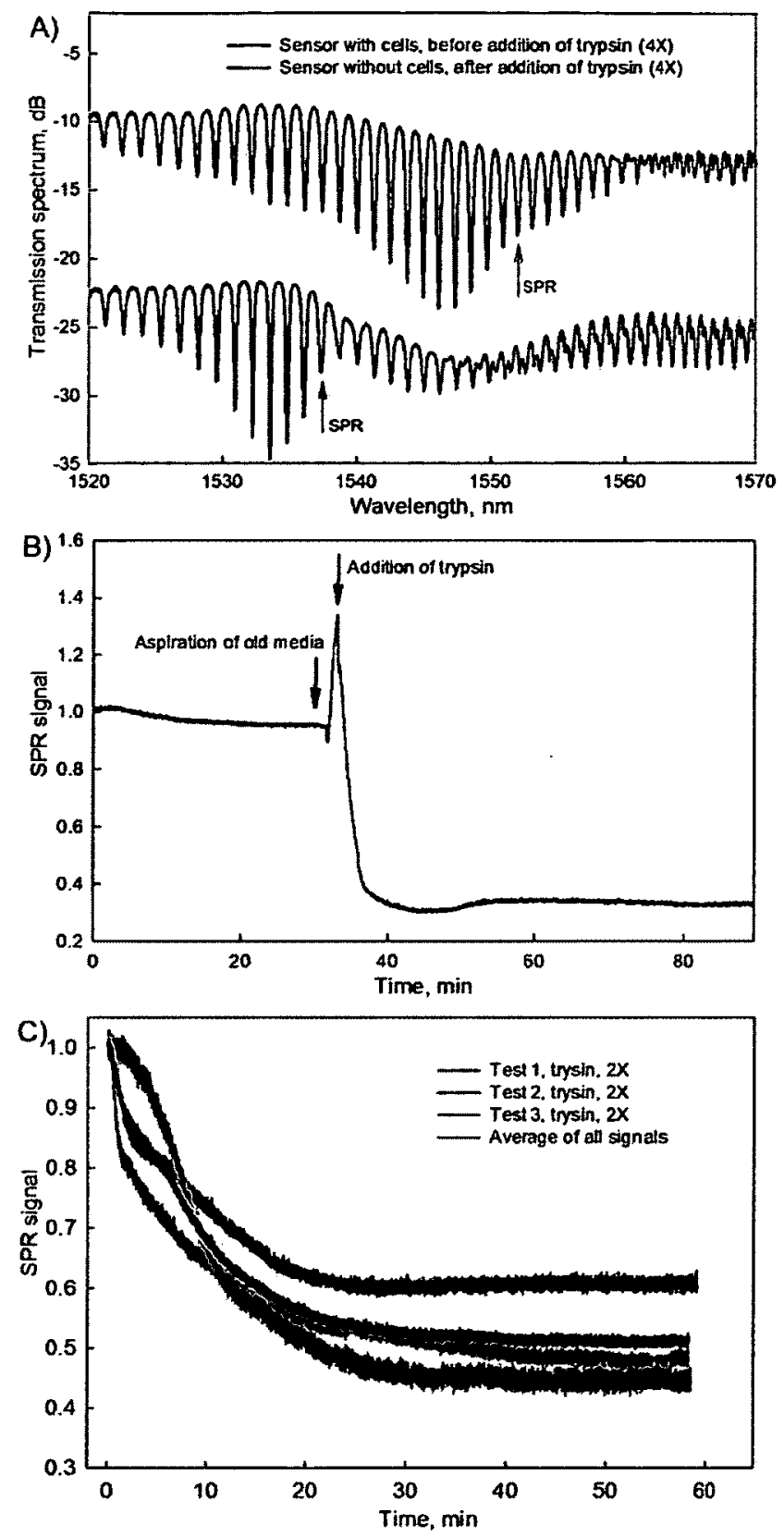

Figure 2. Typical SPR curves obtained with the fiber sensor set-up. (A) SPR-TFBG transmission spectra. The black spectrum is obtained for a sensor with NIH-3T3 cells on its surface. The blue spectrum is from the same sensor after the cells were detached using 
trypsin (4X). Quantitative information is extracted by measuring the amplitude change of the most sensitive resonances indicated by an arrow; (B) Change in the amplitude of the most sensitive SPR resonance following the addition of trypsin (4X); (C) Reproducibility of experiments with trypsin at $2 \mathrm{X}$ concentration. The black curve is the average of the all SPR signals with a confidence interval of $\sigma=0.578$.

Both spectra shown in Figure 2A were obtained with the same SPR-TFBG sensor. The upper response was measured in the presence of cells attached to the sensor surface while the bottom response was measured after cells were detached by trypsin. The difference in the shape of the output signal can be attributed to removal of cells from the fiber resulting in a change in the refractive index at the surface of the sensor. SPR-coupled portions of the spectra responsive to cellular behavior are indicated with arrows pointing at the most sensitive SPR coupled resonances (those resonances that change most strongly to small refractive index changes). The experimental data was analyzed using the method previously presented in Shevchenko et al. $(2010)^{48}$ that is based on tracking the relative amplitude of the most sensitive SPR-coupled cladding mode resonance. The relative change in the amplitude of a resonance is proportional to the SPR effective index change and therefore could be used to evaluate the alterations in refractive index on the sensor surface. Figure 2B shows a typical SPR response during the process of trypsinization. Arrows indicate the moments when the original media surrounding the sensor was aspirated and a fresh solution with trypsin was added. The strong SPR signal changes observed in between these two points is a transient result that reflects the emptying of the Petri dish and can be simply omitted from the data analysis. 
To illustrate the reproducibility of SPR results, three sets of SPR responses were obtained from three different sensors exposed to the same conditions (Figure 2C). All three sensors were seeded with five million NIH-3T3 cells, incubated for $12 \mathrm{~h}$, and exposed to trypsin (2X) solution for 60 minutes. Standard deviation $\sigma$ between the average signal and the three sets of data is 0.0578 indicating the appropriate level of reproducibility of the results.

In addition to seeding sensors with cells and exposing them to a stimulus, it was also possible to regenerate the surface of used sensors using ethanol and trypsin solutions. The sensor surface was regenerated as described in the Experimental section. Exposure to ethanol, trypsin and PBS cleaned the surface from cellular residues and enabled the reuse of same sensors in several experiments.

\section{Detection of Cell Detachment}

Following $12 \mathrm{~h}$ of incubation period with NIH-3T3 cells, the SPR-TFBG sensors were exposed to different concentrations of trypsin solution. Trypsin, a serine protease, is regularly used to cleave cells from the surfaces to which they adhere through destruction of peptide bonds.

Presented in Figure 3A are SPR responses of fiber sensors coated with NIH-3T3 cells and exposed to three different concentrations of trypsin $(0.5 \mathrm{X}, 2 \mathrm{X}$ and $4 \mathrm{X})$. It can be observed from Figure 2C and Figure 3A that SPR signals induced by the addition of trypsin are always negative and saturate within first 20 minutes after of the initial exposure. The amplitudes of the recorded negative SPR signals decrease proportionally to 
the concentration of the applied solution, which is consistent with results reported by Fang et al $2006^{64}$.
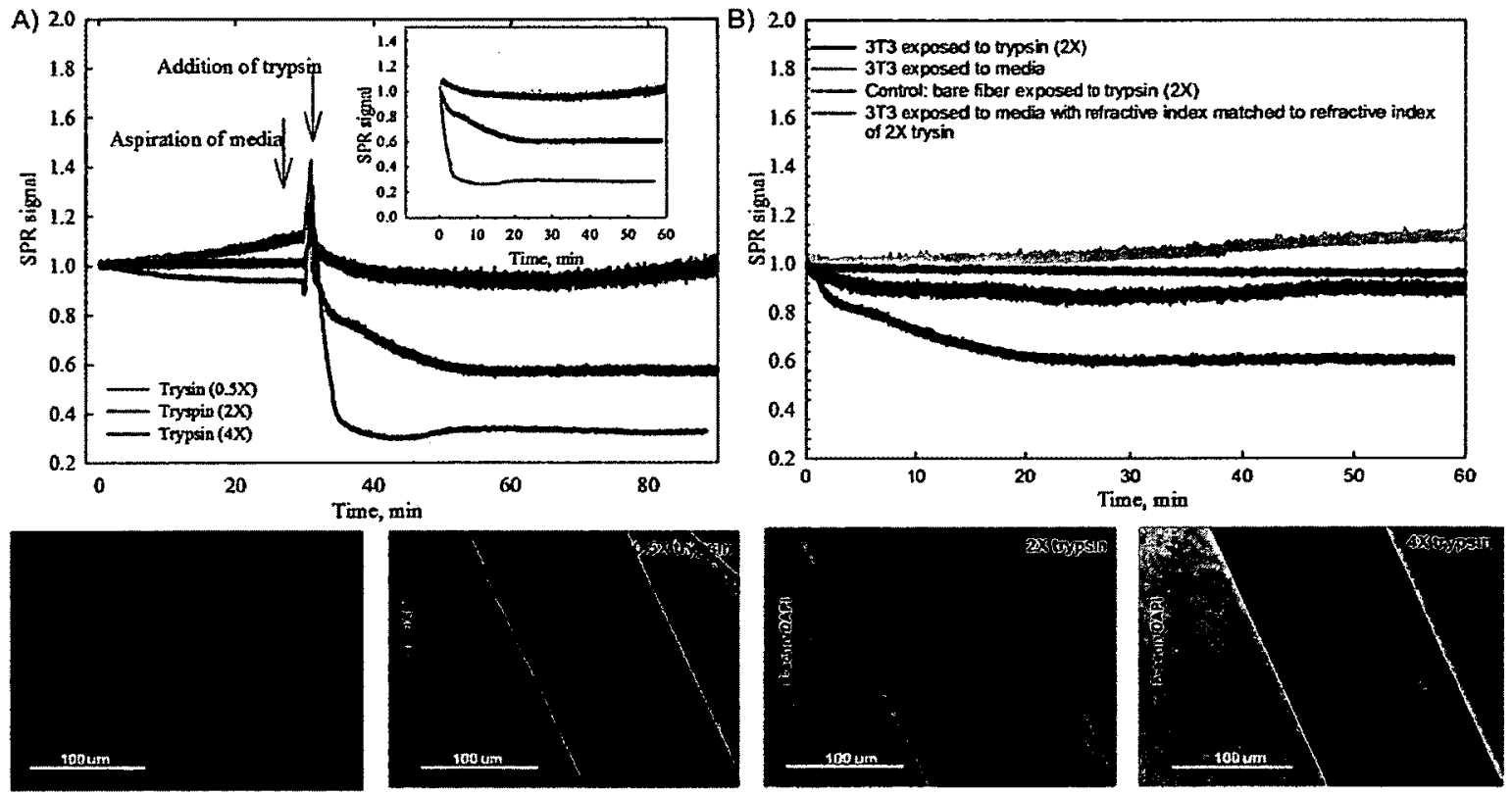

Figure 3. Real-time SPR signals obtained during cellular exposure to trypsin solutions, control SPR measurements, and cytoskeleton/nuclei imaging. (A) SPR responses were measured while NIH-3T3 cells were exposed to trypsin solutions $(0.5 \mathrm{X}, 2 \mathrm{X}$ and $4 \mathrm{X}$ concentrations). Arrows indicate points at which old media was aspirated and the new solution was added. Inset shows SPR responses starting from the moment of addition of stimuli. (B) Various control experiments were performed to monitor SPR responses in the presence and absence of trypsin (2X). (C) Cytoskeleton/nuclei staining (F-actin/DAPI) demonstrates the remaining cells on the sensor surface after exposure to different concentrations of trypsin. 
Figure 3B provides results for a set of SPR controls which illustrates that the measured SPR responses presented in Figure 3A were only due to a reduction in the number of attached cells and not due to other background chemical processes. The SPR response caused by cellular detachment induced by trypsin (navy curve) is compared to the response of a sensor with cells exposed to a regular media (grey curve). As expected, no change in the SPR signal was recorded when standard media was added to the sensor. The violet SPR curve represents the sensor response to media with an adjusted refractive index. In this case, the refractive index of the solution was lowered by the addition of PBS in order to match it to the refractive index of the $2 \mathrm{X}$ trypsin solution. Difference between two responses (dark navy and violet SPR curves) confirms that the measured SPR signal is due to a specific cellular interaction with trypsin solution and is not due to the refractive index mismatch. Additionally Figure $3 \mathrm{~B}$ provides the SPR response of a sensor without cells while being exposed to $2 \mathrm{X}$ trypsin solution (black curve). The difference between a sensor with 3T3 (navy curve) and a sensor without 3T3 (black curve), of which both were exposed to trypsin, proves that the measured SPR signal should be attributed to the change in the number of attached cells and was not affected by the change of the bulk background refractive index induced by the addition of trypsin. The flat response during the control test without cells (black curve) also confirms that there is no non-specific adsorption to the surface of the sensor upon exposure to trypsin. The absence of non-specific interactions proves that the decay in the SPR signal for the sensor with cells exposed to trypsin is not distorted by extracellular non-specific processes. 
The effect of different trypsin concentrations on the degree of cellular detachment was also confirmed with fluorescence staining of cytoskeleton and nuclei. As can be observed in Figure 3C, the density of cellular coverage was affected by the addition of trypsin for all concentrations. A higher concentration of trypsin solution results in lower number of attached cells which correlates well with the real-time SPR data shown in Figure 3A.

\section{Detection of Cellular Uptake}

In addition to the detection of cell detachment the sensor was applied towards monitoring cellular uptake of serum (Figure 4). Fetal Bovine Serum (FBS) is widely used as a supplement for in vitro cell cultures. Usually used at $10 \%(\mathrm{v} / \mathrm{v})$ concentration it provides cells with nutrients required for sustained cell growth and proliferation. In this experiment, FBS was used at concentrations of $10 \%, 30 \%$ and $50 \%(\mathrm{v} / \mathrm{v})$, and was expected to have positive effect on SPR response due to possible cellular uptake of nutrients. 

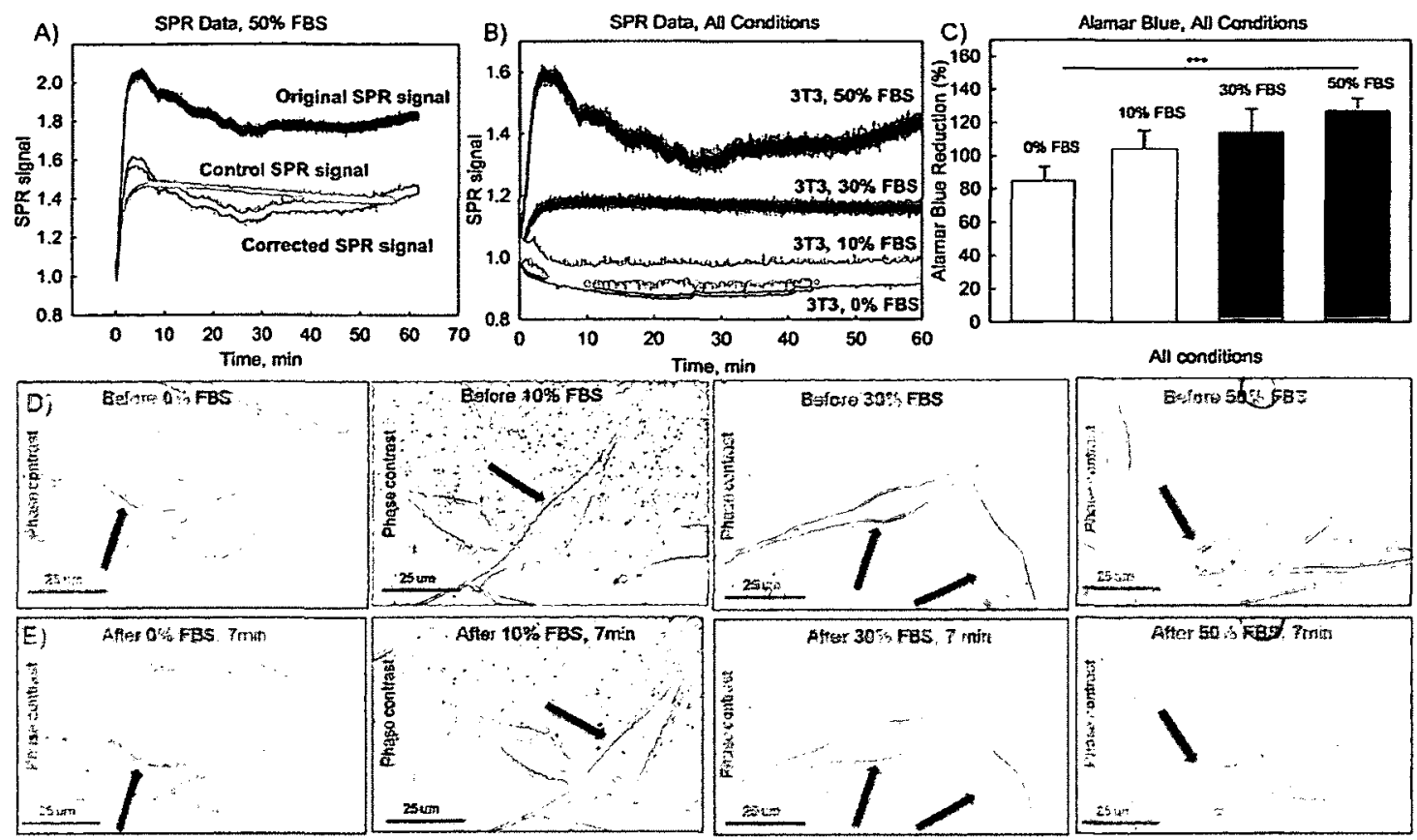

Figure 4. The addition of serum and its cellular uptake monitored in real-time with SPR fiber sensors and confirmed with alamar blue assay and phase contrast imaging. (A) SPR responses during exposure to $50 \%$ (v/v) FBS solution. The black curve is an original SPR signal obtained in the presence of cells, the grey curve is a control SPR signal obtained in the absence of cells. The dark grey curve is the corrected SPR signal obtained from the difference between the original SPR and the control SPR signals. (B) Corrected SPR responses during exposure to different serum solutions $(10 \%, 30 \%$ and $50 \%(\mathrm{v} / \mathrm{v}))$ versus response to media without FBS. (C) Alamar blue assay results for different FBS concentrations; (D-E) Phase contrast images of the NIH-3T3 cells exposed to FBS solutions (50\% FBS, 30\% FBS, 10\%FBS and 0\%FBS). (D) Images were taken before the addition of FBS. (E) Images were taken 7 minutes after the addition of FBS. 
It was found that serum, which contains a substantial number of proteins, resulted in a considerable non-specific adsorption on the surface of the sensors. Figure 4A shows a comparison between original SPR signal measured in the presence of cells exposed to serum and a control SPR signal measured by a sensor without cells, also exposed to the same concentration of serum. The SPR signal increase observed in the case of the sensor without cells is due to non-specific adsorption of proteins from serum on the surface of the sensor. In order to eliminate the contribution from non-specific adsorption, each SPR signal presented on Figure 4B was provided as the difference between the SPR signal measured in the presence of cells and the SPR signal measured in the absence of cells, as illustrated in Figure 4A.

Figure 4B demonstrates cellular responses to $50 \%, 30 \%$ and $10 \%(\mathrm{v} / \mathrm{v})$ FBS solutions. It can be observed that the SPR signal increased within the first five minutes following addition of 50\% FBS (Figure 4A-B). The addition of $30 \%$ of FBS also resulted in a slight positive change. On the contrary, addition of media with $10 \%$ and $0 \%$ of FBS resulted in a slightly negative SPR change (Figure 4B).

Alamar blue assay was also used to evaluate effect of FBS addition on metabolic activity of cells. Results presented in Figure $4 \mathrm{C}$ indicated a consistent increase in the alamar blue fluorescence signal, which was proportional to concentration of applied FBS solution. Additionally, phase contrast images were acquired in order to study the effect of addition of FBS solution on cellular morphology (Figure 4D-E). It should be noted that seven minutes after the addition of $50 \%(\mathrm{v} / \mathrm{v}) \mathrm{FBS}$ on the cells, an increase in the cellular size was observed. There was also a slight increase in the cellular size for $30 \%(\mathrm{v} / \mathrm{v})$ FBS. On the other hand, the addition of media with $10 \%(\mathrm{v} / \mathrm{v})$ FBS and media without FBS did 
not result in any observable change of cellular shapes. Both alamar blue assay and phase contrast imaging data are consistent with obtained SPR results indicating that there is are considerable cellular morphological changes at higher serum concentrations while at lower concentrations the changes are barely detectable.

\section{Detection of Inhibition of Cellular Metabolism}

The study was completed with the addition of an agent that affected cellular metabolism and acted as a toxin. Sodium azide $\left(\mathrm{NaN}_{3}\right)$ is a crystalline salt that is known for inhibition of metabolic activity in mitochondria. By interacting with $\mathrm{Fe}^{3+}$ ions, sodium azide interrupts oxidative phosphorylation and thus inhibits respiration and metabolism 78.

Figure 5A shows the SPR responses measured while NIH-3T3 cells were exposed to two different concentrations of sodium azide $(10 \mathrm{mg} / \mathrm{ml}$ and $2 \mathrm{mg} / \mathrm{ml})$ and media. They are compared with a control SPR response measured in the absence of cells. The measured SPR signature of cellular response to sodium azide exhibits a slow SPR decay. In both cases, the decay in the SPR signal is proportional to the concentration of the applied toxin (sodium azide). As expected, the response to the toxin in the absence of cells and response to media alone are both flat.

Phase contrast images allowed us to observe the changes in cellular morphology upon activation by sodium azide (Figure 5C-D). The phase contrast images compare cells before and 30 minutes after addition of the toxin. A higher concentration of toxin result in a significant reduction in the cellular size. A smaller concentration of sodium azide only slightly affects cellular shape while also causing some cellular shrinkage. No change in cellular morphology was observed in the case of the addition of standard media. 
Additionally, live/dead staining showed that addition of sodium azide results in an increase in the number of dead cells after one hour of exposure to this chemical (Figure $5 \mathrm{E})$.
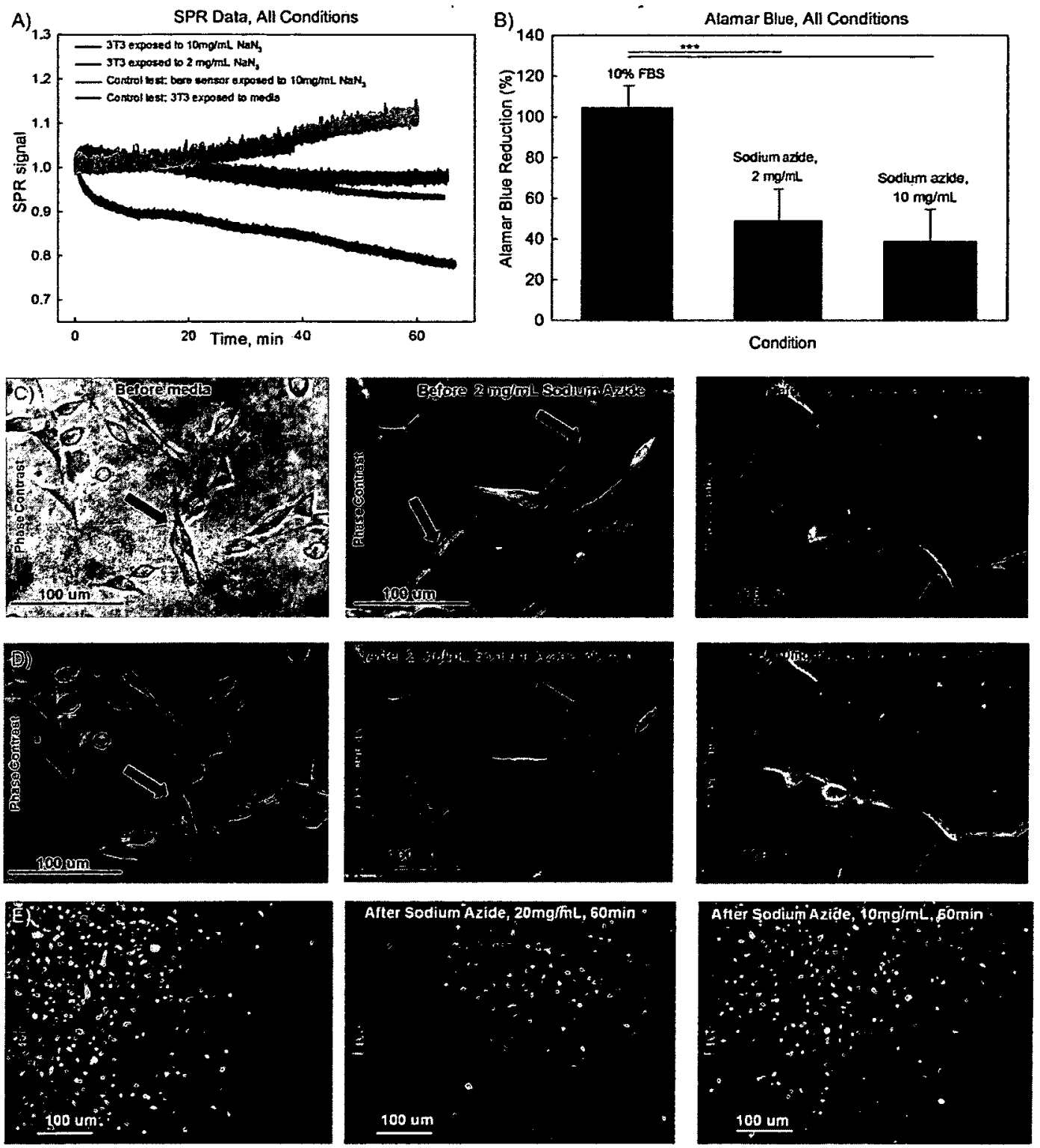

Figure 5. Real-time SPR response of sensors during cellular exposure to sodium azide complemented with alamar blue assay results and imaging confirming effect of the toxin on cellular mortphology and viability. (A) SPR responses during cellular exposure to 
sodium azide $(10 \mathrm{mg} / \mathrm{ml}, 2 \mathrm{mg} / \mathrm{ml})$ and media versus SPR control response to sodium azide $(10 \mathrm{mg} / \mathrm{ml})$ in the absence of cells. (B) Alamar Blue assay results for tested concentrations of sodium azide. (C-D) Phase contrast images of NIH-3T3 cells exposed to sodium azide $(10 \mathrm{mg} / \mathrm{ml}$ and $2 \mathrm{mg} / \mathrm{ml})$ and standard media. (C) Images of cells right before addition of sodium azide. (D) Images of the same cells 30 minutes after addition of sodium azide. (E) Live/dead staining of NIH-3T3 cells exposed to sodium azide $(10 \mathrm{mg} / \mathrm{ml}, 2 \mathrm{mg} / \mathrm{ml})$ and standard media for one hour.

\section{Discussion}

The SPR results present three distinct optical signatures that correspond to very specific cellular responses induced by applied stimuli. SPR signals are generally proportional to refractive index changes on the surface of the sensor. When coated with NIH-3T3 fibroblast cells, SPR-TFBG sensors measure a local refractive index change that is strongly influenced by the density of cells attached to the surface and also to reactions within the attached cells, which lead to changes in the mean refractive index of the cell interior. The $1 \mathrm{~cm}$-long fiber biosensor has a sensitive surface area of $3.92 \mathrm{~mm}^{2}$ and a significant depth of penetration of the plasmonic field which is approximately 1500 $\mathrm{nm}$. As a result, the measured response is an average of the responses from all the cells attached to its sensing area. The density of cells attached to the fiber surface, changes of the cellular morphology or changes of intracellular content have considerable effects on the sensor response as the combination of these factors determine the average refractive index seen by the plasmon wave over its propagation length along the fiber. 
The optical signature measured during cellular detachment induced by trypsin was a negative SPR change with semi-exponential character (Figure $3 \mathrm{~A}$ ). The signal change was consistently dose-dependent and was not caused by refractive index mismatch or by non-specific adsorption, as proved by various controls (Figure 3B). As reported by Strasser et al. (2011) ${ }^{79}$ the refractive index of intracellular content is on average higher than that of media, thus a decrease of the number of attached cells should result in a decrease in the effective SPR index. The observed negative SPR signal change confirms the anticipated refractive index decrease and is correlated well with fluorescence images showing a reduction of the number of attached cells.

The optical signature measured during exposure of cells to FBS demonstrated the opposite character, it was positive with a very rapid saturation time (5-10 min). The rapid SPR increase in the case of $50 \%$ and $30 \%(\mathrm{v} / \mathrm{v})$ FBS could be attributed to the cellular uptake of nutrients from the added solution and their subsequent spreading. Phase contrast images confirm that there is a slight increase of the cellular shapes right after addition of FBS solutions at $30 \%$ and $50 \%(\mathrm{v} / \mathrm{v})$ concentrations. The absence of positive SPR signal change after addition of $10 \%$ and $0 \%(v / v)$ FBS could be explained by the fact that right before the addition of a stimulus the sensors were immersed in media solution with the $10 \%(\mathrm{v} / \mathrm{v})$ concentration of FBS, thus cells did not react to the same or lower concentration of serum. The decrease in the SPR signal after the addition of media without FBS could be caused by cellular uptake of freshly added media and subsequent intracellular refractive index decrease.

The addition of sodium azide also produced a distinct optical signature (Figure 5A) that can be characterized by an SPR signal decay within 20 minutes. The recorded 
negative decay of SPR signal is consistent with the SPR signature of sodium azide reported by Chabot et al ${ }^{4}$. Phase contrast imaging indicates that there was a significant change in the cellular shape due to exposure to sodium azide at both concentrations. Live/dead fluorescence staining shows that there is also a consistent increase in the number of dead cells after exposure to sodium azide. Alamar blue assay confirms that there is a reduction in the metabolic activity after exposure to sodium azide that correlates with the SPR data. All results are making apparent the immediate effect of sodium azide that is expressed through a negative SPR signal change, a decrease of metabolic activity and an increase in the number of dead cells. Thus it can be concluded that the observed SPR signature is representative of immediate cellular response to a toxin, which results in an inhibition of the metabolic activity and cellular death.

All three stimuli, trypsin, FBS, and sodium azide, have had different effects on NIH-3T3 fibroblast cells and resulted in distinguishable optical signatures that are reflective of cellular changes. While exposure to FBS produced the smallest SPR signal change, trypsin caused the strongest negative SPR signal change. SPR-TFBG sensors having several tens of cladding resonances and as a result of it, a wide operating range, are an appropriate choice for tracking such big chemical changes. At the same time, a wide operating range does not compromise the sensitivity of the sensor. The high sensitivity $\left(500 \mathrm{~nm} /\right.$ refractive index units ${ }^{45}$ ) allows for the measurement of intracellular changes of smaller magnitudes, such as those obtained in the case of serum uptake that could be barely visualized using standard imaging tools. In the future, if necessary, a slightly different interrogation setup reported by Caucheteur et al $2011^{51}$ may enable the detection of even finer intracellular changes that could be triggered inside cells ensuring 
that the sensor platform can be employed for a wide spectrum of triggered intracellular responses.

\section{Conclution}

This paper presents the findings from one of the first fiber biosensors for real-time analysis of cellular behavior. The SPR-TFBG sensor is a compact and robust fiber biosensor that is manufactured out of a standard telecommunication single-mode fiber coated with a thin layer of gold and interrogated with traditional fiber optic device measurement instrumentation. The presented sensor was the first fiber sensor to be completely integrated into cell incubator equipment. To enable cell adhesion to the surface of the fiber, the sensors were coated with fibronectin. It was followed by incubation of sensors with NIH-3T3 fibroblast cells and they were exposed to different stimuli, namely, trypsin, FBS and sodium azide, producing three distinct optical signatures. Trypsin cleaved attached cells from the fiber sensors resulting in a sharp decrease of the SPR signal. On the other hand, the addition of serum resulted in a sharp increase of the SPR signal, which could be caused by the uptake of nutrients from the serum solution. The addition of sodium azide resulted in a slow SPR signal decay that could be explained by the inhibition of cellular metabolism upon penetration of sodium azide inside the cells. In all cases the measured cellular response was immediate and the amplitude of SPR change was proportional to the concentration of applied stimuli. Responses to all stimuli were compared to the set of controls where sensors were exposed to solutions without stimuli, or where only stimuli were applied to sensors in the absence of cells. Rigorous control experiments enabled the confirmation of measured SPR signals 
resulting from a specific cellular response to the added stimuli. It was also proven that surface of the sensors can be regenerated for repeated use.

Overall, the results illustrate that it is possible to use the presented fiber biosensor technology to characterize a wide range of cellular responses right from the moment of stimulation. The information is obtained in real-time and the whole measurement method is label-free, providing a unique platform for non-invasive monitoring of cellular behavior. Furthermore, this sensor technology has the potential to be used in the characterization of less pronounced and more complex cellular reactions, such as for triggering very specific signaling pathways that would be challenging to detect using other analytical tools.

\section{Acknowledgements}

This project was supported by the National Institutes of Health (HL092836; HL099073; AR057837; DE019024; EB008392; GM095906), the National Science Foundation (DMR0847287), the Institute for Soldier Nanotechnologies, the US Army Corps of Engineers, the Natural Sciences and Engineering Research Council of Canada, the Canada Research Chairs program, and the Canadian Institute for Photonic Innovations.

\section{ABBREVIATIONS}

SPR, Surface Plasmon Resonance; TFBG, Tilted Fiber Bragg Grating; FBS, Fetal Bovine Serum. 


\section{Chapter 6}

\subsection{Discussion}

SNR: As it was mentioned in Chapter 1, SNR is a function of the signal strength and the noise level. It was concluded that the main source of noise in the applied interrogation setup was the laser source that was built into the Micro Optics Si720 interrogation unit. Its noise level has been quite consistent throughout all experiments. Experiments with the detection of DNA self-assembly and detection of alpha human thrombin as presented in Chapter 4 illustrate that the noise level can be substantial compared with the signal strength and therefore results in low SNR. To improve SPR signal quality, data averaging was applied in order to smooth the signal and get rid of the noise. However, in the case with the monitoring of cellular reactions (Chapter 5), the amplitude of the measured refractive index change was significantly bigger. Even though the interrogation system had the same noise level, the SNR was no longer an issue and there was no need to smooth the data. Thus it can be concluded that applications focused on detecting biomolecular interactions or other chemical reactions that would not induce strong bulk refractive index change require SPR-data post-processing such as filtering or smoothing of the measured signal. Another solution could be to change the interrogation unit and to employ a more stable light source.

SPR polarization selectivity: As it was shown in Chapter 2, SPR excitation on the surface of a metal coated fiber is highly sensitive to the polarization state of the incident 
light. It was shown that it is possible to alter the strength of SPR coupling by rotating the vector of the polarization. This sensitivity can be explained by the fundamental dependency of SPR on the polarization state of the light used to excite it. For instance, it is known that in planar structures the TM mode is primarily responsible for SPR excitation while TE mode cannot be coupled into SPR. However in the case of SPR excitation on the surface of a metal coated optical fiber we cannot consider the TE or TM modes simply because fiber cladding is a circular waveguide and it supports more complicated mode structures (hybrid modes). Nevertheless, the employed TFBG allows excitation of cladding modes with various combinations of electro-magnetic components that provides an opportunity for polarization sensitivity. The rotation of linearly polarized light reaching the grating determines which cladding modes are going to be excited through TFBG.

Although it is difficult to determine the relative orientation of the light polarization reaching the TFBG and the TFBG structure itself, it is possible to control coupling to SPR and minimize polarization losses. Control over coupling can be achieved by rotating half-wave and quarter wave plates in polarization controller therefore aligning polarization vector with respect to TFBG orientation.

A setup involving use of polarized light is extremely sensitive to the fiber alignment. After an experiment has been started it is important to minimize fiber movement and bending over sharp edges that could cause change of the polarization of light that interacts with a TFBG.

Once certain cladding modes have been excited it is not possible to alter polarization content of these modes by changing the environment in which sensor is 
immersed. Excitation of modes and their polarization content is only determined by the relative alignment of the TFBG and the incident light polarization.

Limitations of the SPR-TFBG sensor configuration: this research revealed the advantages and limitations associated with the SPR-TFBG setup. Some of the limitations are as follows:

1) Even though chemical sensitivity is considerably high ( $450-550 \mathrm{~nm} / \mathrm{RIU})$, the SNR can be an issue in applications that involve a very small refractive index change, such as the detection of DNA self-adsorption;

2) The quality of the gold coating has a direct influence on the SPR coupling. By impacting the width of SPR coupled region; as well as, the amplitudes of the resonances of the coupled modes. The variation between gold coatings of different sensors can result in measurable differences in spectra possibly leading to reproducibility issues;

3) In situ bio-chemical measurement require a microfluidic platform that can provide a controlled environment and consistency between experiments. Taking measurements without such a custom-designed platform might have a tremendous effect on the reproducibility of the obtained data;

4) Polarization sensitivity provides an opportunity to fine-tune SPR coupling; however it also makes the fiber setup vulnerable to such effects as fiber bends and movement. It is important to keep the fiber straight and stationary in order to maintain the same polarization throughout the experiment. This can be an issue for in-field applications if long fibers are used for connecting interfacing the sensor element;

5) Considerable depth of penetration of plasmonic field has been taken into consideration when developing an application for the SPR-TFBG sensor. While it is an 
advantage for applications like measurement of cellular responses where range of refractive index changes is considerable, it can be a limitation as in applications like detecting DNA self-adsorption where thickness of the adsorbed monolayer is only few nm; and

6) Sensor's reuse is possible with the current configuration; however experiments illustrated that the gold coatings deteriorate after 4 to 5 experiments. It is recommended to explore other metal coatings that can be more stable and thus allow for more usages.

\subsection{Summary}

In this thesis a plasmonic fiber sensor has been studied and its application in several different areas was explored.

The sensor element presented here was first reported in Shevchenko's thesis ${ }^{67}$. The sensor works through the excitation of the SPR effect on its surface. The excitation is accomplished with the help of a TFBG imprinted in the core of a singlemode optical fiber. The planes of the grating are oriented at a slight angle $\left(6^{\circ}-10^{\circ}\right)$ relative to the normal of the fiber's axis, granting access to several tens of cladding modes the same time. If a fiber with a TFBG is coated with a thin metal layer and the phase matching condition for SPR is satisfied, then cladding modes can be coupled into SPR. All of the excited claddin modes potentially could be coupled into SPR, providing this particular sensor configuration with a wide operating range (0.1 RIU). Shevchenko's 
master's thesis reported on the original discovery of the SPR-TFBG coupling mechanism and attempted the first characterization of its chemical sensitivity.

Research presented in this thesis is built upon the previously proposed sensor configuration; however it delves deeper into the optimization of the sensor setup, developing data analysis, and more importantly applying the sensor in chemical and biological sensing.

The work presented here begins with a fundamental discovery into the mechanism behind SPR excitation and develops a method of analysis that can ensure the successful application of the sensor for measuring various bio-chemical processes. This research milestone consisted of studying in great detail the coupling of light into a plasmonic wave, as presented in Chapter $2^{49}$. These findings illustrate that SPR-TFBG sensors are highly sensitive towards the orientation of the polarization of the light used to excite SPR. One can optimize the coupling of cladding modes into SPR by rotating the angle of polarization of the linearly polarized light at the input of the sensor. The attained control over the SPR coupling by adjusting polarization allows for tuning and maximizing of SPR coupling, thus producing a better SPR signal, a narrower SPR resonance and a smaller SNR.

The coupling of cladding modes into SPR alters the transmission spectrum, making it more complex than the transmission spectrum of a bare TFBG. The SPR-TFBG response overlaps the regular TFBG spectrum with a set of cladding mode resonances that are coupled to SPR. Amplitudes of SPR-coupled resonances must be monitored in order to extract information about chemical changes taking place on the surface of the fiber. Work presented here consists of matching experimental data with theoretical 
findings to provide insight into the way the spectral response of the sensor is formed. The FIMMWAVE mode solver from Photon Design was used to identify all cladding modes that could be coupled into SPR for a certain fiber sensor configuration. Theoretical findings helped to find out that SPR-coupled modes have a significant imaginary component and that they occur at the same wavelengths as those resonances with smallest amplitudes in the measured SPR-TFBG spectra. This helped to identify that part of the spectrum is SPR-coupled and should be monitored for the purpose of extracting information about changes occurring on the surface of the sensor.

Identification of the SPR-coupled resonances and the centre of the SPR were followed by the development of a data analysis technique specific to this particular sensor. Applications involving the monitoring of chemical changes (such as adsorption of sub-nm thick polymer layers or DNA monolayer) required the development of a data analysis approach that would permit monitoring of hardly detectable spectral changes with the lowest SNR. The chosen analysis method consists of the tracking the amplitude changes of the most sensitive SPR-coupled resonance. Such analysis grants the sensor a high refractive index resolution of $4 * 10^{-5}$, which proven to be critical in biomolecular applications.

The breakthrough in our understanding of the interaction mechanism between the TFBG and the SPR has made possible the advancements associated with the sensor applications that are reported in chapters 3,4 , and 5 .

Chapter 3 presents the application of the SPR-TFBG towards monitoring of the process of the formation of thin polymer layers. SPR-TFBGs were used towards real-time monitoring of the process of adsorption of 30 monolayers deposited in a step-by step 
process. SPR data revealed the characteristics of the polymer adsorption process, providing the data necessary for the study of the dynamics during formation each of the monolayers. The adsorbed polymer structure was later studied using AFM, which provided the means to identify the thickness of the deposited polymer layer. SPR response was combined with theoretical data which provided the opportunity to evaluate the studied process and the performance of the sensor. The combination of SPR response with a theoretical model revealed an opportunity for multiparameter characterization of the polymer coating ${ }^{48}$.

Chapter 4 presents an application of SPR-TFBG sensor towards biosensing. The sensors'. surface was functionalized with a monolayer of aptamers that served as biorecognitiuon elements. Following this, the functionalization sensor was applied to the detection of target molecules of thrombin. Results indicate that the sensor can be used to detect thrombin-like targets at concentrations in between $0.1 \mu \mathrm{M}-5 \mu \mathrm{M}$ in buffer and serum. The sensor was alsoused to characterize affinity between interacting biomolecules. SPR data was supported with fluorescence imaging and AFM in order to confirm the SPR findings ${ }^{30}$.

Chapter 5 presents the application of the SPR-TFBG towards cellular analysis. For this particular application, the SPR-TFBG sensor element was integrated into a Petri dish and the entire sensor interrogation unit was setup to operate with a cell culture incubator. The surface of the sensor was modified with a layer of fibronectin protein which ensured cellular adhesion to the surface of the gold coating. The sensor was then incubated with fibroblast cells for 12 hours, and following this the sensor was applied to measure the cellular response of the attached fibroblast cells. Cells adhered to the surface of the 
sensor were exposed to three different stimuli that induced three distinct responses. Studied cellular processes were cellular detachment, cellular uptake of nutrient and cellular death induced by a toxin. The SPR response obtained in experiments with cells was complemented with a set of control responses aimed to eliminate background processes such as non-specific adsorption occurring on the surface of the sensor. Final SPR data was confirmed with imaging and spectroscopy data, revealing the possibility to study extra- and intra- cellular processes right after their instigation in a real-time and label-free regime ${ }^{66}$. In this particular application, the sensors' capacity to measure extraand intra-cellular reactions is attributed to its high sensitivity, significant depth of penetration of plasmonic field inside sensed media and its wide operating range.

All explored applications were aimed at measuring vastly different chemical and biological processes by taking advantage of the sensor's various characteristics. The development of the polarization optimization of SPR coupling, the shifting the operating range towards aqueous solutions, the development of an analysis method for interpretation of fine spectral changes and the development of custom experimental platforms for biochemical sensing ensured the successful utilization of the SPR-TFBG sensor in each of these applications. The attained performance of this sensor is comparable not only to the performance of other fiber sensors but also to the performance of state of the art analytical platforms, illustrating its potential for real-life applications. 


\subsection{Future Work Recommendations}

There are several directions for future research that can be recommended in order to further the work of the SPR-TFBG sensor. They are categorized in four groups and presented below in the order of deceasing importance:

\section{Improvement of the quality of metal layer and sensor's manufacturing} techniques:

Reproducibility is one of the major issues preventing commercialization of analytical and sensor technologies. Reproducibility of the SPR-TFBG sensor results (similar to results generated by any other SPR sensor) strongly depends on the quality of the metal layer and its uniformity around circumference of the fiber. Furthermore, roughness due to variability in the thickness of the metal around its circumference increases the SPR width, thus lowering the SNR.

1) Sensors manufactured for this project were subject to electron beam evaporation and sputtering deposition techniques. It is recommended that other metal coating techniques are explored with the aim of enhancing the uniformity of deposited coatings and improving control over surface roughness.

2) It is recommended to explore other kinds of metals and possibly combination of several different metals (or semiconductors) with the purpose of gaining more control over SPR excitation and SPR output parameters.

\section{Development of a more sophisticated data analysis approach:}

Due to the complexity of the sensor output signal, the SPR-TFBG sensor requires a custom-designed data analysis solution. The data analysis technique applied in this work 
was based on the relative amplitude change and was mostly derived from the experimental data. It is recommended that the existing data analysis approach is improved by assimilating more of theoretical findings in order to confirm spectral changes occurring during biochemical reactions (or any other reaction to be studied). Moreover, the development of data analysis should be done while sensor's performance is evaluated using another analytical tool. The sensor analytical tool should serve as a reference and allow for referenced monitoring of the SPR-TFBG response. It should be mentioned that the resolution and SNR performance of the reference analytical tool should be at least at the same level as the performance of the SPR-TFBG sensor. SPR Biacore or QCM platforms are well-known analytical platforms that exhibit a very high performance level and therefore could be used for referencing.

\section{Further miniaturization and integration of the element sensor:}

One of the advantages of the developed sensor is its potential to be used to measure biochemical processes in situ. In order to explore in situ applications, it is important to design a sensor platform that can ensure control over sensed processes and the timely delivery of targets/reagents.

1) The sensor element could be integrated with a microfluodic platform that would reduce the volume of the sensed media to be delivered, and would thus allow for overall control over delivery of analyzed substances.

2) The current sensor configuration relies on TFBG working in transmission; it is recommended that a reflection regime is explored for the purpose of miniaturization of the sensor element. Fiber sensor working in reflection (i.e. a fiber probe) would simplify the sensor configuration and would thus allow for a simpler experimental routine. 
3) It is recommended that several sensing elements are integrated so that they can work in a parallel. Such a configuration can allow for parallel measurements of the same process; it could be applied for the monitoring of various cellular processes if sensor elements are integrated in a Petri dish. If sensor elements are integrated to work in parallel and functionalized by using different biorecognition elements, they could be applied for the detection of several analytes in the same environment. On another side, if one of the sensor elements is not functionalized, then its readings could be used as a control response.

4) The integration of a sensor element into a portable device can also be pursued. Further engineering work is needed in order to integrate a sensor element with some kind of interrogation setup so that the final product can be a compact and hand-held device.

\section{Biorecognition schemes and surface modification approaches:}

Finding appropriate biorecognition schemes for each specific application is as important as the developing the sensor element itself. It is important to match the SPRTFBG sensor with a biorecognition scheme that can take advantage of the sensors' strengths, such as its wide operating range and big depth of penetration of plasmonic field within the sensed media.

1) In order to take advantage of the big depth of penetration of plasmonic field into the sensed media, it is recommended that the development of biorecognition schemes similar to immunoassays is attempted. The adsorption of several biomolecular layers and the amplification of the detected target signal with nanoparticles or biomolecules can provide the opportunity to reduce the LOD to femtomolar and picomolar concentrations. However, it should be remembered that the utilization of sandwich assay biorecognition 
schemes makes the detection of analyte labeled and complicates the whole procedure of analyte detection. It might not be suitable for applications where the sensor would be packaged in a hand-held device and used outside of a laboratory setting.

2) Another approach that could help decrease the LOD and keep the sensing modality label-free is to increase the surface area of the sensor. This could be accomplished by increasing the surface roughness of the metal coating, by depositing nanoparticles ${ }^{80}$ or by depositing a 3D matrixes on a surface of the metal. In these cases increased surface area results in bigger overlap between plasmonic field and receptor-analyte conjugates thus increasing SNR. Use of nano-engineered sensor surfaces can also help with elimination of non-specific interactions and increase of specific sensitivity ${ }^{81,82}$.

3) It is important that a method to regenerate the surface of the gold-coated sensor is found in order to improve sensor's longevity. Sensors applied in the cellular sensing project $^{66}$ (Chapter 5) were regenerated with the help of trypsin and buffer solutions. The application of trypsin ensured the cleavage of cells from the sensor's surface, such surface treatment was proven to be sufficient for that particular application. However this method might not be effective for other applications, especially those that deal with detection of biomolecular targets at small concentrations and might require more rigorous control over sensor's surface quality.

It is also recommended that the combination of plasmonics with other optical effects such as SERS, Coherent anti-Stokes Raman spectroscopy (CARS), spectroscopy and fluorescence is explored. By combining different sensing mechanisms, a variety of different functionalities can be achieved. For example, combining plasmonics with fluorescence can provide the opportunity for multi-target or multi-parameter analyte 
detection and the achievement of smaller LODs. Combining plasmonics with CARS ${ }^{83}$ can provide the opportunity for very sensitive detection (CARS generally has smaller LOD than SPR). Combining plasmonics with both CARS and spectroscopy can provide the opportunity to detect analyte with, or even without the aid of a small biorecognition element.

In summary, a combination of the plasmonic effect with other optical effects can result in new disruptively improved sensor performance with a higher sensitivity and an improved functionality. Matching of the sensor element with an appropriate biorecognition scheme will help with leveraging of the sensor's fundamental properties such as sensitivity and resolution that are predetermined by the employed sensing mechanism while having direct influence on the LOD and specificity. And finally, integration of the sensor element with other optical components, sensors and a microfluidic platform can ensure successful application of the sensor in a chosen domain. 


\section{References (Chapter 1, 5 and 6):}

1. M. Hide, T. Tsutsui, H. Sato, T. Nishimura, K. Morimoto, S. Yamamoto, K. Yoshizato, Analytical Biochemistry 2002, 302. 28-37, DOI: 10.1006/abio.2001.5535. 2. Y. Yanase, H. Suzuki, T. Tsutsui, T. Hiragun, Y. Kameyoshi, M. Hide, Biosensors and Bioelectronics 2007, 22. 1081-1086, DOI: 10.1016/j.bios.2006.03.011. $3 . \quad$ R. Robelek, Bioanalytical Reviews 2009, 1. 57-72, DOI: 10.1007/s12566-009$0005-y$.

4. V. Chabot, C. M. Cuerrier, E. Escher, V. Aimez, M. Grandbois, P. G. Charette, Biosensors and Bioelectronics 2009, 24. 1667-1673, DOI: 10.1016/j.bios.2008.08.025. 5. Y. Fang, A. M. Ferrie, G. Li, Biochimica et Biophysica Acta (BBA) - Molecular Cell Research 2006, 1763. 254-261, DOI: 10.1016/j.bbamcr.2006.01.006.

6. Y. Fang, A. M. Ferrie, BMC Cell Biology 2007, 8. 24, DOI: 10.1186/1471-21218-24.

7. D. H. R. J. J. Scibek, T. A. Bunch, Y. Fang, A. G. Frutos. Corning Inc. , 2012, vol. 2012, p 17.

8. Y. Fang, A. Ferrie, FEBS Letters 2008, 582. 558-564, DOI:

10.1016/j.febslet.2008.01.021.

9. S. Kurosawa, H. Aizawa, M. Tozuka, M. Nakamura, J.-W. Park, Measurement Science and Technology 2003, 14. 1882-1887.

10. L. F. Kourkoutis, J. M. Plitzko, W. Baumeister, Annual Review of Materials Research 2012, 42.33-58.

11. S. Ekgasit, F. Yu, W. Knoll, Sensors and Actuators B: Chemical 2005, 204. 294301.

12. P. P. Prasad, INTRODUCTION TO BIOPHOTONICS. John Wiley \& Sons, Inc. : United States of America, 2004; p 618.

13. D. Lorenser, X. Yang, R. W. Kirk, B. C. Quirk, R. A. McLaughlin, D. D. Sampson, Optics Letters 2011, 36. 3894-3896.

14. W. U. Dittmer, T. H. Evers, W. M. Hardeman, W. Huijnen, R. Kamps, P. de Kievit, J. H. M. Neijzen, J. H. Nieuwenhuis, M. J. J. Sijbers, D. W. C. Dekkers, M. H. Hefti, M. F. W. C. Martens, Clinica chimica acta; international journal of clinical chemistry 2010, 411. 868-73.

15. P. J. Scully, L. Betancor, J. Bolyo, S. Dzyadevych, J. M. Guisan, R. FernándezLafuente, N. Jaffrezic- Renault, G. Kuncová, V. Matějec, B. O'Kennedy, O. Podrazky, K. Rose, L. Sasek, J. S. Young, Measurement Science and Technology 2007, 18. 3177-3186. 16. H. Tao, J. J. Amsden, A. C. Strikwerda, K. Fan, D. L. Kaplan, X. Zhang, R. D. Averitt, F. G. Omenetto, Advanced materials (Deerfield Beach, Fla.) 2010, 22. 3527-31. 17. A. J. Steckl, Nature Photonics 2007, 1. 3-5.

18. F. S. Ligler, Analytical Chemistry 2009, 81. 519-526.

19. A. P. F. Turner, Science 2000, 290.1315-1317

20. D. R. Thevenot, K. Toth, R. A. Durst, G. S. Wilson, Biosensors and Bioelectronics 2001, 16. 121-131. 
21. E. Snitzer, J. W. Hicks, Program of the 1959 Annual Meeting of the Optical Society of America 1959, 49. 1128.

22. K. C. Kao, B. S. Eng, D. Ph, G. A. Hockham, B. S. Eng, I. E. E. Graduate, Electrical Engineers, Proceedings of the Institution of 1966, 113.1151-1158, DOI: 10.1049/piee.1966.0189.

23. C. Incorporated. Corning Incorporated 2002, vol. 2012, p Product INformation

24. L. Yuan, Y. Dong, Journal of Intelligent Material Systems and Structures 2008, 20. 809-813.

25. T. Chen, D. Xu, M. Buric, M. Maklad, P. R. Swinehart, K. P. Chen, Measurement Science and Technology 2010, 21. 094036-8.

26. M. Fischer, M. Wahl, G. Friedrichs, Biosensors \& bioelectronics 2012, 33. 172178.

27. V. V. R. Sai, T. Kundu, C. Deshmukh, S. Titus, P. Kumar, S. Mukherji, Sensors and Actuators B: Chemical 2010, 143. 724-730.

28. A. Hassani, M. Skorobogatiy, Journal of the Optical Society of America B 2009, 26. 1550.

29. Y. Yanase, A. Araki, H. Suzuki, T. Tsutsui, T. Kimura, K. Okamoto, T. Nakatani, T. Hiragun, M. Hide, Biosensors and Bioelectronics 2010, 25: 1244-1247, DOI:

10.1016/j.bios.2009.09.042.

30. Y. Shevchenko, T. J. Francis, D. A. D. Blair, R. Walsh, M. C. DeRosa, J. Albert, Analytical Chemistry 2011, 83.7027-7034, DOI: 10.1021/ac201641n.

31. A. Khetani, V. S. Tiwari, A. Harb, H. Anis, Optics Express 2011, 19. 1524415254.

32. D. U S, C. Y. Fu, K. S. Soh, B. Ramaswamy, A. Kumar, M. Olivo, Biosensors \& bioelectronics 2012, 33. 393-398.

33. R. H. Ritchie, Physical Review 1957, 106. 874-881.

34. D. Li, C. Z. Ning, Optics Express 2011, 19. 14594-14603.

35. F. S. Ligler; C. A. R. Taitt, Optical Biosensors: Present and Future. 2002.

36. J. Homola, Y. S. Sinclair, G. Gunter, Sensors and Actuators B 1999, 54. 3-15.

37. I. Abdulhalim, M. Zourob, A. Lakhtakia, Electromagnetics 2008, 28. 214-242.

38. a. J. C. Tubb, F. P. Payne, R. B. Millington, C. R. Lowe, Sensors and Actuators B: Chemical 1997, 41.71-79.

39. R. Alonso, J. Subias, J. Pelayo, F. Villuendas, J. Tornos, Applied Optics 1994, 33. 5197-5201.

40. A. Wang, A. Docherty, B. T. Kuhlmey, F. M. Cox, M. C. J. Large, Optics Letters 2009, 34. 3890-3892.

41. L. Obando, D. Gentleman, J. Holloway, K. Booksh, Sensors and Actuators B: Chemical 2004, 100. 439-449.

42. M.-H. Chiu, S.-F. Wang, R.-S. Chang, Optics Letters 2005, 30. 233-235.

43. B. Spackova, M. Piliarik, P. Kvasnicka, C. Themistos, M. Rajarajan, J. Homola, Sensors and Actuators B 2009, 139. 199-203.

44. H. Y. -J., L. Y. -L., H. J. -F., Journal of the Optical Society of America B 2006, 23. $801-811$.

45. Y. Y. Shevchenko, J. Albert, Optics Letters 2007, 32. 211-213.

46. T. Allsop, R. Neal, C. Mou, P. Brown, S. Saied, S. Rehman, K. Kalli, D. J. Webb, J. Sullivan, D. Mapps, I. Bennion, Applied Optics 2009, 48. 276-286. 
47. G. Nemova, R. Kashyap, Journal of Materials Science: Materials in Electronics 2007, 18. 327-330.

48. Y. Shevchenko, N. U. Ahamad, A. Ianoul, J. Albert, Optics Express 2010, 18. 20409-20421.

49. Y. Shevchenko, C. Chen, M. a. Dakka, J. Albert, Optics Letters 2010, 25. 637-9.

50. L.-Y. Shao, Y. Shevchenko, J. Albert, Optics Express 2010, 18. 11464-71.

51. C. Caucheteur, Y. Shevchenko, L. Y. Shao, M. Wuilpart, J. Albert, Optics Express 2011, 19. 1656-1664.

52. J. Vial, A. Jardy, Analytical Chemistry 1999, 71. 2672-2677.

53. P. J. Conroy, S. Hearty, P. Leonard, R. J. O'Kennedy, Seminars in cell \& developmental biology 2009, 20.1-26.

54. T. Fodey, P. Leonard, J. O'Mahony, R. O'Kennedy, M. Danaher, Trends in Analytical Chemistry 2011, 30.254-269.

55. V. Velusamy, K. Arshak, O. Korostynska, K. Oliwa, C. Adley, Biotechnology advances 2010, 28. 232-254.

56. G. Köhler, C. Milstein, European Journal of Immunology 1976, 6. 511-519.

57. E. J. Cho, J.-W. Lee, A. D. Ellington, Annual review of analytical chemistry 2009, 2. 241-264.

58. P. Dua, S. Kim, D.-K. Lee, Methods (San Diego, Calif.) 2011, 54. 215-225. 59. P.-H. Lin, R.-H. Chen, C.-H. Lee, Y. Chang, C.-S. Chen, W.-Y. Chen, Colloids and surfaces. B, Biointerfaces 2011, 88. 552-558.

60. E. Torres-Chavolla, E. C. Alocilja, Biosensors \& bioelectronics 2009, 24. 3175 82.

61. T. Mairal, V. C. Ozalp, P. Lozano Sánchez, M. Mir, I. Katakis, C. K. O'Sullivan, Analytical and bioanalytical chemistry 2008, 390. 989-1007.

62. X. He, S. McMahon, R. Rasooly, Biocatalysis and Agricultural Biotechnology 2012, 1. 105-109.

63. Label-Free Biosensors: Techniques and Applications Cambridhe University Press: New York, NY, USA, 2009.

64. Y. Fang, A. M. Ferrie, N. H. Fontaine, J. Mauro, J. Balakrishnan, Biophysical Journal 2006, 91.1925-1940, DOI: 10.1529/biophysj.105.077818.

65. S. B. Kim, H. Bae, J. M. Cha, S. J. Moon, M. R. Dokmeci, D. M. Cropek, A. Khademhosseini, Lab on a Chip 2011, 11. 1801-7, DOI: 10.1039/c1lc20098d.

66. Y. Shevchenko, G. Camci-Unal, D. F. Cuttica, M. R. Dokmeci, J. Alberta, A. Khademhosseini. Analytical Chemistry (submitted) 2012.

67. Y. Shevchenko. Surface Plasmon Resonance Sensor Based on the Tilted Fiber Bragg Grating. Carleton University, Ottawa, ON, Canada, 2007

68. F. J. S. J. A. Ferguson, D. R. Walt, Analytical Chemistry 2000, 72. 5618-5624.

69. H. S. Jang, K. N. Park, C. D. Kang, J. P. Kim, S. J. Sim, K. S. Lee, Optics Communications 2009, 282. 2827-2830, DOI: 10.1016/j.optcom.2009.03.078.

70. T. C. F. Y. Ruan, S. Warren-Smith, P. Hoffmann, R. C., H. E.-H. Moore, T. M. Monro, Optics Express 2008, 16. 18514-18523.

71. W. J. B. M. Smietana, P. Mikulic, A. Ng, R. Chinnappan, M. Zourob, Optics Express 2011, 19.7971-7978.

72. A. François, J. Boehm, S. Y. Oh, T. Kok, T. M. Monro, Biosensors and Bioelectronics 2011, 26. 3154-3159, DOI: 10.1016/j.bios.2010.12.018. 
73. A. Khademhosseini, R. Langer, J. Borenstein, J. P. Vacanti, Proceedings of the National Academy of Sciences of the United States of America 2006 103. 2480-2487.

74. A. M. Ghaemmaghami, M. J. Hancock, H. Harrington, H. Kaji, A.

Khademhosseini, Drug discovery today 2012, 17. 173-181.

75. S. B. Kim, H. Bae, K. I. Koo, M. R. Dokmeci, A. Ozcan, A. Khademhosseini, Journal of laboratory automation 2012, 17. 43-9, DOI: 10.1177/2211068211426695.

76. B. D. G. R. A. K. Sharma, Journal of Optics A: Pure and Applied Optics 2007, 9. 682-687, DOI: 10.1088/1464-4258/9/7/020.

77. D. F. Mosher, Annual Review Medicine 1984, 35. 561-575.

78. T. Ishikawa, B. L. Zhu, H. Maeda, Toxicology and Industrial Health 2006, 22. 337-341.

79. S. D. Strasser, G. Shekhawat, G. D. Rogers, V. P. Dravid, A. Taflove, V. Backman, Optics Letters 2011, 37. 506-508.

80. N. Nagase, K. Terao, N. Miyanishi, K. Tamai, N. Uchiyama, T. Suzuki, H. Takao, F. Shimokawa, F. Oohira, Analyst 2012, 137. 5034-5040.

81. S. R. Raz, G. R. Marchesini, M. G. E. G. Bremer, P. Colpo, C. P. Garcia, G. Guidetti, W. Norde, F. Rossi, Analyst 2012, 137. 5251-5259.

82. O. Krushinitskaya, T. I. Tønnessen, H. Jakobsen, E. A. Johannessen, Journal of Membrane Science 2011, 376. 153-161.

83. C. Steuwe, C. F. Kaminski, J. J. Baumberg, S. Mahajan, Nano Letters 2011, 11. 5339-5343. 


\section{Author's Contributions}

[1] Shevchenko, Y., C. Chen, et al. (2010). "Polarization-selective grating excitation of plasmons in cylindrical optical fibers." Optics Letters 25(5): 637-639.

The research presented was mainly a collaborative effort between Y.S. and J.A. J.A. defined the objectives while Y.S was responsible for establishing the experimental setup, carrying out the data analysis and the subsequent interpretation of the findings. M.D. worked with Y.S on the simulations and C.C. worked with J.A. on the theoretical analysis. All authors have contributed to, seen and approved the manuscript.

[2] Shevchenko, Y., N. U. Ahamad, et al. (2010). "In situ monitoring of the formation of nanoscale polyelectrolyte coatings on optical fibers using Surface Plasmon Resonances." Optics Express 18 (19): 20409-20421.

The research presented here was carried out in collaboration between J.A., Y.S. and A.I. on outlining paper's objectives, setting up experiment and troubleshooting. Y.S. was responsible for a data analysis, interpretation, simulations and drafting of the paper. N.U.A. was working with Y.S. and A.I on conducting the experiment with deposition of polyelectrolyte while A.I. and N.U.A. were responsible for AFM imaging. All authors have contributed to, seen and approved the manuscript. 
[3] Shevchenko, Y., T. J. Francis, et al. (2011). "In Situ Biosensing with a Surface Plasmon Resonance Fiber Grating Aptasensor." Analytical Chemistry 83(18): 70277034.

The research presented here was carried out in collaboration between all authors. Y.S. worked together with J.A and M.C.D. on the main concept and its implementation. Y.S contributions include setting up experimental work, data collection, its analysis, and preparation of the manuscript. T.F. and D.D.B. worked with Y.S on conducting the experiments with detection of DNA self-adsorption and detection of analyte as well as worked with M.C.D on confocal imaging and preparation of biological samples for all experiments. All authors have contributed to, seen and approved the manuscript.

[4] Shevchenko, Y., G. Camci-Unal, et al. (2012). Surface Plasmon Resonance Fiber Sensor for Real-Time and Label-Free Monitoring of Cellular Behavior. Analytical Chemistry (submitted).

The research presented in the manuscript was a result of collaboration between all authors. Y.S. was responsible for proposing of the project and worked with A.K., G.C.U., J.A. on outlining of the project's objectives and its implementation. G.C.U., D.F.C. and Y.S. were involved in the laboratory experiments, troubleshooting and interpretation of the results. Y.S. was responsible for the data analysis. A.K., M.D., G.C.U., Y.S. and J.A. were involved in the interpretation of results, revising and finalizing of the manuscript. All authors have contributed to, seen and approved the manuscript. 


\section{Author's Research Output (2007-2012)}

\section{Publications in Peer-Reviewed Journals:}

1. Y. Shevchenko, C. Chen, M. a. Dakka, and J. Albert, "Polarization-selective grating excitation of plasmons in cylindrical optical fibers," Optics Letters 25, 637-639 (2010).

2. Y. Shevchenko, N. U. Ahamad, A. Ianoul, and J. Albert, "In situ monitoring of the formation of nanoscale polyelectrolyte coatings on optical fibers using Surface Plasmon Resonances," Optics Express 18, 20409-20421 (2010).

3. L.-Y. Shao, Y. Shevchenko, and J. Albert, "Intrinsic temperature sensitivity of tilted fiber Bragg grating based surface plasmon resonance sensors," Optics Express 18, 11464-11471 (2010).

4. C. Caucheteur, Y. Shevchenko, L. Y. Shao, M. Wuilpart, and J. Albert, "High resolution interrogation of tilted fiber grating SPR sensors from polarization properties measurement," Optics Express 19, 1656-1664 (2011).

5. Y. Shevchenko, T. J. Francis, D. A. D. Blair, R. Walsh, M. C. DeRosa, and J. Albert, "In Situ Biosensing with a Surface Plasmon Resonance Fiber Grating Aptasensor," Analytical Chemistry 83, 7027-7034 (2011).

6. Y. Shevchenko, G. Camci-Unal, D. F. Cuttica, M. R. Dokmeci, J. Alberta, and A. Khademhosseini, "Surface Plasmon Resonance Fiber Sensor for Real-Time and Label-Free Monitoring of Cellular Behavior," (Analytical Chemistry (submitted) 2012). 


\section{List of Abbreviations}

AFM: Atomic Force Microscope

BSA: Bovine serum albumin

CARS: Coherent anti-Stokes Raman spectroscopy

DNA: Deoxyribonucleic acid

ELISA: Enzyme-linked immunosorbent assay

FBG: Fiber Bragg Grating

LOD: Limit of Detection

LPG: Long Period Grating

NIH-3T3: National Institute of Health 3T3 (fibroblast cell line)

PBS: Phosphate buffered saline

PDADMAC: Polydiallyl Dimethyl Ammonium Chloride

PSS: poly sodium-4-styrenesulfonate

QCM: Quartz Crystal Microbalance

RIU: Refractive Index Unit

SELEX: Systematic Evolution of Ligands by Exponential Enrichment

SEM: Scanning Electron Microscope

SERS: Surface enhanced Raman spectroscopy

SMF-28: Single mode fiber (full name Corning® $\mathrm{SMF} 28^{\mathrm{TM}}$ )

SNR: signal to noise ratio

SPR: Surface Plasmon Resonance

TFBG: Tilted Fiber Bragg Grating 


\section{Glossary}

Affinity: attraction of molecules for each other.

Analyte: a substance or chemical constituent that is of interest in an analytical procedure.

Antibody: a protein produced by the body's immune system when it detects harmful substances, called antigens.

Antigen: a substance that evokes the production of one or more antibodies.

Aptamer: an oligonucleotide sequence identified by in vitro selection (SELEX) from a random sequence library of deoxyribonucleotides or ribonucleotides.

Aptasensor: biosensor that uses aptamers as a biorecognition element.

Biorecognition element: biomolecule on the surface of the sensor responsible for interacting with target molecules and changing one of the properties (thickness, refractive index of the biorecognition layer, generation of fluorescent signal) that is being monitored by the sensor.

Cytoskeleton staining/actin staining: staining of the cell inner content (actin organelle structures) using fluorescent phalloidins.

Fibernectin: a kind of extracellular matrix protein that can be used to facilitate cell adhesion to surfaces.

Fibroblast: connective tissue cell responsible for secretion of extracellular matrix and collagen.

FIMMWAVE: a generic, fully vectorial mode finder for $2 \mathrm{D}+\mathrm{z}$ waveguide structures, which may be made of any material and of almost any geometry. 
Immunoassay: a kind of biochemical test aimed to detect analyte with help of several kinds of buiomolecules. Some of these biomolecules are antibodies.

Label-free analysis: an analysis that does not require any kind of label (radioisotope, fluorophore, nanoparticle) for detection of the target.

Specificity or selectivity: capability of a biosensor to detect presence of the analyte and distinguish it from other biomolecules presented in same solution. 